\title{
Seychelles: Second Review Under the Stand-By Arrangement, Request for Waivers of Nonobservance of Performance Criteria, and Financing Assurance Review-Staff Report; Press Release on the Executive Board Discussion; and Statement by the Executive Director for Seychelles
}

In the context of the second review under the Stand-By Arrangement, request for waivers of nonobservance of performance criteria, and financing assurance review for Seychelles, the following documents have been released and are included in this package:

- $\quad$ The staff report for the Second Review Under the Stand-By Arrangement, Request for Waivers of Nonobservance of Performance Criteria, and Financing Assurance Review, prepared by a staff team of the IMF, following discussions that ended on May 18, 2009, with the officials of Seychelles on economic developments and policies. Based on information available at the time of these discussions, the staff report was completed on June 16, 2009. The views expressed in the staff report are those of the staff team and do not necessarily reflect the views of the Executive Board of the IMF.

- $\quad$ A Press Release summarizing the views of the Executive Board as expressed during its June 30, 2009, discussion of the staff report that completed the review.

- $\quad$ A statement by the Executive Director for Seychelles.

The documents listed below have been or will be separately released.

Letter of Intent sent to the IMF by the authorities of Seychelles *

Memorandum of Economic and Financial Policies by the authorities of Seychelles *

Technical Memorandum of Understanding*

*Also included in Staff Report

The policy of publication of staff reports and other documents allows for the deletion of marketsensitive information.

\author{
Copies of this report are available to the public from \\ International Monetary Fund • Publication Services \\ $70019^{\text {th }}$ Street, N.W. • Washington, D.C. 20431 \\ Telephone: (202) 623-7430 • Telefax: (202) 623-7201 \\ E-mail: publications@imf.org •Internet: http://www.imf.org
}

\section{International Monetary Fund Washington, D.C.}





\title{
INTERNATIONAL MONETARY FUND
}

\section{SEYCHELLES}

\section{Second Review Under the Stand-By Arrangement, Financing Assurances Review, and Modification of Performance Criteria}

\author{
Prepared by the African Department \\ (In consultation with other Departments)
}

Approved by Roger Nord and Aasim Husain

June 16, 2009

SDR 7.1 million has been disbursed to date under the two-year Stand-By Arrangement (SBA) (SDR 17.6 million; 200 percent of quota), approved on November 14, 2008.

SDR 0.88 million is available upon completion of the second review.

Discussions were held in Victoria May 4-18, 2009. Staff met with President James Michel, Minister of Finance Danny Faure, Governor Pierre Laporte of the Central Bank of Seychelles (CBS), other senior government officials, and representatives of the private sector, civil society and the diplomatic community. The mission was headed by Paul Mathieu, and included Nikoloz Gigineishvili, Patrick Imam (all AFR), Nkunde Mwase (SPR), and Magdalena Polan (MCM).

The program is on track and macroeconomic stabilization has advanced rapidly. The authorities are implementing the program with determination and a high degree of ownership. All quantitative performance criteria (PCs) at end-March 2009 were met. One of two structural benchmarks for end April was missed, reflecting capacity constraints in issuing new bank capitalization norms, but was completed in late May.

The program has been updated to reflect the progress in macro stabilization, but also the impact of global recession and heightened piracy. The revised program profile locks-in a significant portion of the fiscal over performance of the first quarter, while allowing for some additional spending in priority areas. At the same time, the tighter fiscal stance and rapid disinflation will permit a measured easing of monetary policy to support lending and private sector growth. The authorities are requesting modification of the quantitative performance criteria.

Seychelles has made major progress in its public debt restructuring strategy. In mid-April Paris Club creditors granted exceptional treatment under the Evian approach. Good faith discussions with other creditors are being pursued and the authorities are in compliance with the Fund's lending into arrears policy.

Program design: In light of the progress on macroeconomic stabilization and significantly reduced risks to the program, it is proposed that the program reviews be put on a semi-annual basis. Performance criteria, financing assurances reviews, and purchases would remain on a quarterly basis.

The authorities continue to make strong progress in addressing the areas of concern identified in the safeguards assessment, including a new central bank act that meets international best practice. 


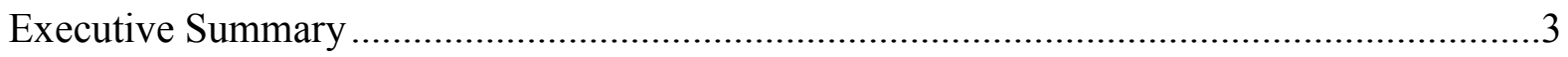

I. Macroeconomic Developments and Performance Under the Program ...............................4

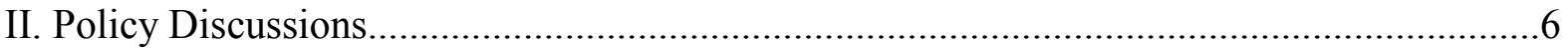

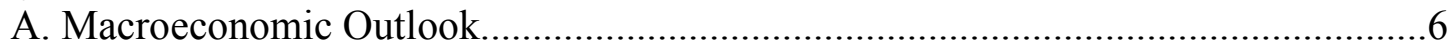

B. Fiscal Policy ............................................................................................... 7

C. Monetary, Exchange and Financial Sector Policies ........................................... 7

D. External Sector, Financing, and Debt Sustainability ..........................................

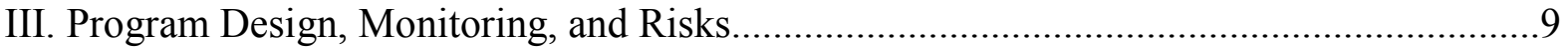

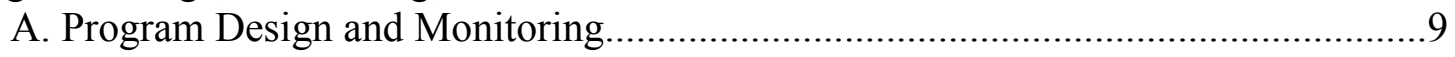

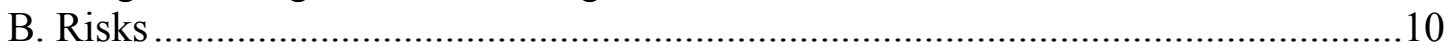

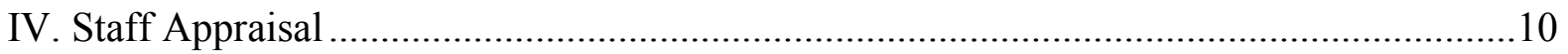

Tables

1. Selected Economic and Financial Indicators, 2006-10 ..............................................12

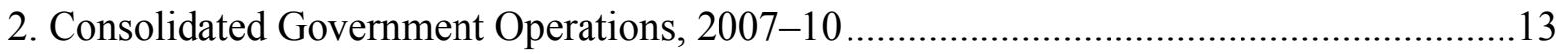

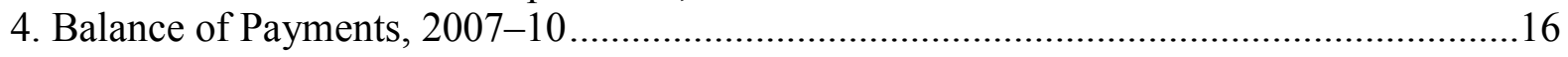

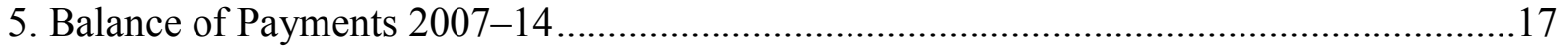

6. Projected Financing Requirements and Sources, 2008-19 .........................................18

7. Financial Soundness Indicators for the Banking Sector, 2007-09 ..................................19

8. Fund Disbursements and Timing of Reviews Under the Two-Year Stand-By

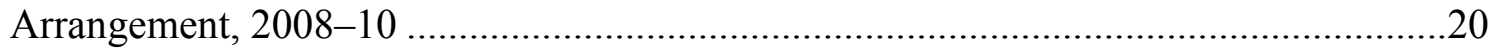

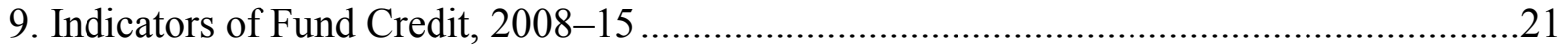

Figures

1. Seychelles: Evolution of Monetary and Exchange Rates, 2008 ......................................5

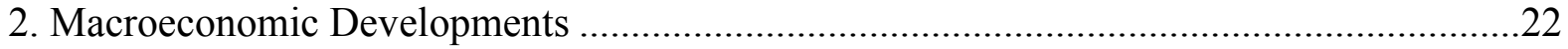

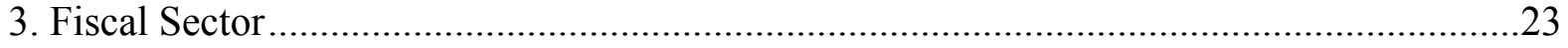

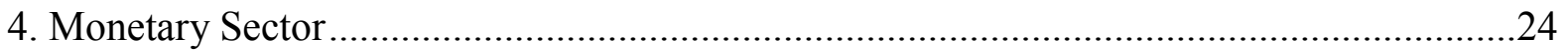

Appendices

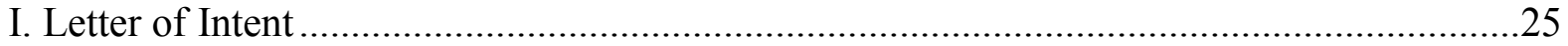

Attachment I. Memorandum of Economic and Financial Policies for 2009 ....................26

Attachment II. Technical Memorandum of Understanding ........................................39

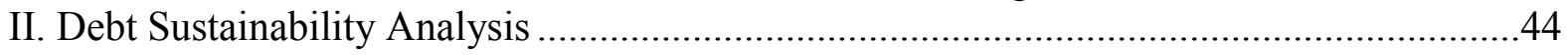




\section{EXECUTIVE SUMMARY}

- Macroeconomic performance in the first four months of 2009 was more favorable than anticipated in the program. CPI inflation was reduced markedly and interest rates followed a sharp downward trend from a peak early in the year. After stabilizing, the nominal exchange rate appreciated strongly against the U.S. dollar. government revenue was more buoyant and the primary surplus was well above target making possible a large repayment of domestic public debt. The capitalization of the financial system has improved and remains adequate.

- However, Seychelles is being hard hit by global recession and real GDP is expected to decline 11 percent in 2009. While tourism is faring better than expected, construction is down sharply on delays in FDI projects and industrial output is off significantly. In addition, heightened piracy activity in and around Seychelles' territorial waters is affecting the tuna industry as well as shipping and pleasure sailing.

- Structural reforms are being implemented with determination. The initial steps of a profound structural reform are being put in place, notably on a fundamental reform of tax policy and a strengthening of governance and monitoring of the parastatal sector.

- The program has been updated to reflect the progress in macro stabilization, but also the impact of global recession and heightened piracy. The revised program profile aims to lock in a significant portion of the fiscal over performance of the first quarter. The tighter fiscal stance and rapid disinflation will permit a measured easing of monetary policy to support lending and private sector growth.

- $\quad$ Program financing gaps are higher for 2009-10, reflecting the impact of piracy on tuna exports. These gaps are expected to be closed by additional program grant support from the EU and through the public external debt restructuring.

- $\quad$ Staff recommends completion of the second review under the SBA. All quantitative PCs were observed and the structural reform program remains on track.

- As the risks to the program have decreased significantly, reflecting the major progress in macroeconomic stabilization, staff proposes that the program monitoring revert to semiannual reviews. Quarterly performance criteria and financing assurances review would remain. 


\section{Macroeconomic Developments And Performance Under the Program}

1. The authorities are implementing the reform program with determination and rapid progress has been made in macroeconomic stabilization. CPI inflation has declined sharply to the low single digits in the first four months of 2009 (annualized). Tracking the disinflation, interest rates have been on a downward trend (from about 30 percent in December to 12.4 percent in late-May on 91 day T-bills) and the nominal exchange rate has appreciated significantly against the U.S. dollar since its low in December 2008 (Figure 1). The strong policy performance has created a virtuous cycle environment for fiscal and monetary policy with falling budget financing costs and stronger demand for money.

2. However, GDP is now projected to decline by $\mathbf{1 0 . 7}$ percent in 2009 (Table 1). ${ }^{1}$ While the decline in tourism is more moderate than earlier expected, construction is down sharply on delays in FDI hotel projects and industrial output has fallen. In addition, increased piracy activity off the east coast of Africa, adjacent and into Seychelles' territorial waters is affecting the tuna catch, and to a lesser extent also tourism. In response, the EU and others are bolstering their security aid in the region. Official unemployment statistics have risen to the mid single digits. ${ }^{2}$

\section{This performance was anchored by a large fiscal over performance in the first} quarter (Table 2). Government revenue was more buoyant than expected and the primary surplus was well above target, helped by the large on-lending repayment by the national oil company (SEYPEC). Expenditures were below target on the impact of the tighter procurement procedures under the new law and the non use of the public financial institutions recapitalization contingency. The public financial institutions are doing much better than had been feared, on balance sheet effects of the depreciation, administrative cost savings, and lower markets risks than feared on the back of the rapid financial stabilization. As a result, domestic public debt has fallen by 4 percent of GDP and the interest bill will be reduced significantly going forward.

4. The Central Bank of Seychelles (CBS) has been implementing with success the new market-based monetary policy to control reserve money. Monetary policy was kept tight and progress has been made in improving the regulatory environment, with ongoing Fund TA support (Table 3). Nominal interest rates have declined sharply but they remain high in real terms (10 percent). Bank portfolios are weathering the impact of higher market and credit risk and bank capitalization has improved. A new central bank act that meets international best practice was approved by parliament in late April.

\footnotetext{
${ }^{1}$ The program incorporates revised official national accounts statistics for 2005-06 and provisional data for 2007-08 based of improvements in methodology and data coverage. These data show somewhat higher levels and different growth rates.

${ }^{2}$ However, official data do not fully capture unemployment and the authorities are working to improve coverage.
} 
5. All quantitative PCs for end-March 2009 have been met with margins (MEFP; Table 1). One end-April structural benchmark was missed due to the delay in approval of new bank capitalization norms, which was implemented in late May (MEFP, Table 2). Good progress was achieved toward the end-June structural benchmarks and the structural PC is expected to be met. ${ }^{3}$ Other structural reforms are on track.

Figure 1. Seychelles: Evolution of Monetary and Exchange Rates, 2008

The exchange rate has appreciated recently from its depreciated level following the float.

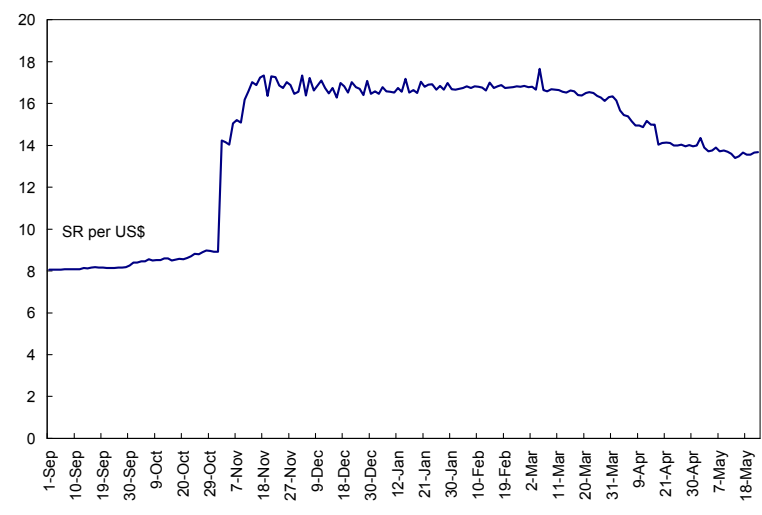

Monetary conditions have remained tight...

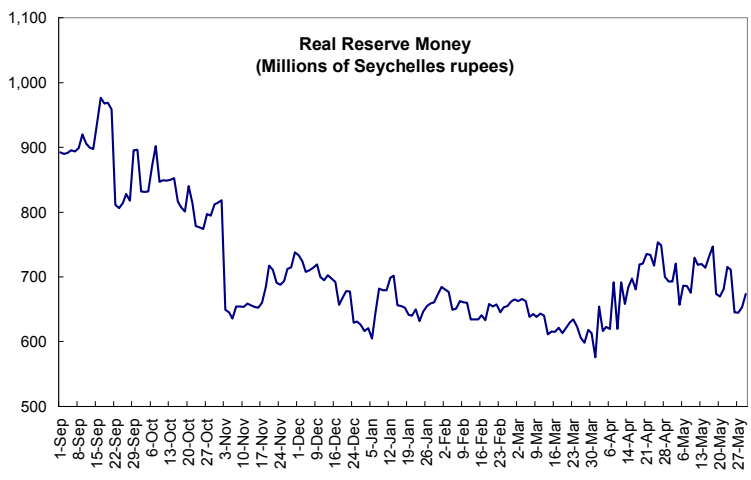

Spreads have been erratic but on a downward trend as market institutions develop.

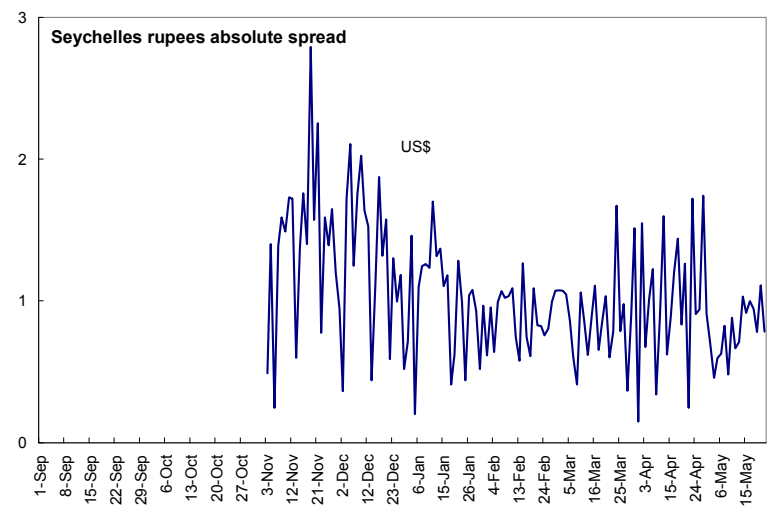

... and market-based interest rates on 91-day T-bills have eased off their highs.

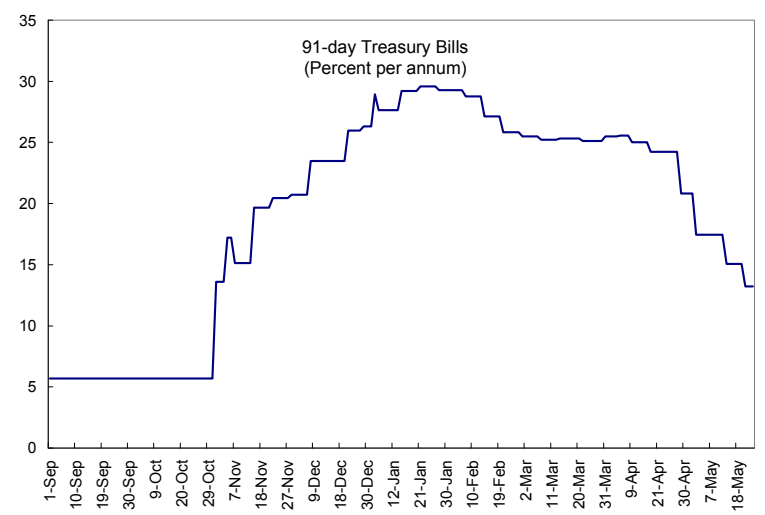

Sources: Central Bank of Seychelles and IMF staff estimates.

\footnotetext{
${ }^{3}$ Following discussion with the staff on the new Fund policy on structural PCs, the authorities decided not to seek conversion of the end-June structural PC to a benchmark, reflecting their strong commitment to meeting the target.
} 
6. The external current account deficit widened sharply in 2008, reaching 46 percent of GDP, mainly reflecting a large surge in imports in the fourth quarter (Table 4). Despite the decrease in world food and oil prices together with a slowdown in tourism and FDI, imports rose, primarily reflecting repressed demand released with the elimination of foreign exchange restrictions. Imports appear to have been largely financed by foreign currency outside the banking system and other capital flows. Imports declined sharply in the first quarter of 2009 and gross official reserves rose above the program target (to 1 month of imports) on some one-off inflows and the unblocking of foreign asset accounts.

\section{On April $16^{\text {th }}$, Paris Club creditors granted exceptional debt treatment to} Seychelles under the Evian approach. The agreement resulted in a clearance of US $\$ 140$ million of arrears. ${ }^{4}$ Seychelles' debt stock will be reduced by 45 percent in nominal terms in two tranches. ${ }^{5}$ The remaining amounts will be rescheduled over 18 years, including five years of grace. No payments will be due in 2009 other than a "goodwill" payment of US\$1 million by end-June, with very limited payments due in the next few years as moratorium interest will be partially deferred. The authorities have shared relevant data with the creditors, including the cash flow simulation following the Paris Club agreement, and estimations of financing gaps and necessary debt service reduction. The Paris Club agreement has given an impetus to the debt restructuring discussions with other creditors, including bondholders and banks.

\section{Policy Discussions}

\section{A. Macroeconomic Outlook}

8. The program has been updated to reflect the progress in macro stabilization, but also the impact of global recession and heightened piracy. The revised program profile aims to lock in a significant portion of the fiscal over performance of the first quarter, while allowing for an easing in the rest of the year to address some areas of priority spending. At the same time, the tighter fiscal stance and rapid disinflation will permit a measure easing of monetary policy to support lending and private sector growth. Inflation is expected to remain in the low single digits. The external current account deficit is projected to decline markedly, with a rise in official reserves to 1.3 months of imports. Focus is now shifting to implement an important structural transformation of the economy, notably on a fundamental reform of tax policy and addressing underperformance and quasi-fiscal risks in the parastatal sector.

\footnotetext{
${ }^{4}$ Including owed to South Africa.

${ }^{5}$ The first tranche is conditioned on the completion of the $2^{\text {nd }}$ SBA review and the second tranche on the completion of the first review under a successor arrangement (expected around June 2010) and satisfactory implementation of the comparability of treatment clause. South Africa, a non Paris Club member and major creditor, participated in the negotiation.
} 


\section{B. Fiscal Policy}

9. The over performance of the first quarter, and the associated virtuous financial dynamics, has created fiscal space for priority expenditure, notably to combat piracy and raise social spending for the remainder of the year (MEFP, \17). While the primary surplus target has been raised to 11.4 percent of GDP, this reflects the large overperformance in the first quarter, together with a measured loosening in the last three quarters. The fiscal program will benefit from much lower interest costs. . Revenue is expected to remain on track, despite the real GDP decline, in part reflecting the impact of the depreciation of the rupee on turnover, supported by the widened tax base and continuing measure to strengthen administration. On the expenditure side, strengthen commitment procedures, notably through the new Procurement Act and tightened financial controls, will reduce inefficiencies and contain outlays on goods and services. As the public financial institutions are doing reasonably well, the need for their potential recapitalization is significantly lessened, and the budget contingency for this purpose has been cut back.

10. The authorities are launching a fundamental tax reform in June (MEFP, $\uparrow 18$ ). The medium-term strategy involves a modernization of the tax regime to eliminate sectoral preferences and exemptions, harmonize tax rates, and remove distortions and inefficiencies. A new business tax code and a personal income tax will be introduced in 2010 , followed by a VAT by 2012. Major upgrades to tax administration are also in process, supported by continued technical assistance from the Fund.

\section{The program also features a major reinforcement of financial control and} governance oversight of state enterprises (MEFP, ๆ19-21). External financial and management audits of the largest public enterprises are in process, which will inform the action plans for the necessary reform of the sector. A strategic assessment of the role of each public entity is underway. Implementation of a treasury single account is well underway and is leveraging efforts to strengthen budget control. With the support of the World Bank, a Public Expenditure Review (PER), focusing on the health and education sectors, has been launched to reinforce expenditure control and rationalization.

\section{Monetary, Exchange and Financial Sector Policies}

\section{In line with its strengthened mandate under the new CBS act, monetary policy} will focus on achieving price stability. With inflation in low single digits and demand pressures subsided, the program includes a measured easing of monetary policy through a gradual reduction in reserve requirements (initially to 12 percent in July) and a further reduction in the local asset ratio to boost credit growth. ${ }^{6}$ In addition, starting from April, the

\footnotetext{
${ }^{6}$ While the original reserve money targets have been retained, implementation of the treasury single account will create additional space under the targets, as about 1 percent of GDP in public entity deposits are anticipated to be repatriated to the treasury single account at the CBS in the coming months. The local asset ratio is much like a secondary reserve requirement and has been reduced from 60 percent in 2008 to 40 percent in April.
} 
constitution of required reserves on foreign currency deposits will gradually shift to foreign currency. At the same time, policy interest rates will need to remain positive in real terms to anchor price stability, and the CBS is prepared to act promptly at the first signs of reemergence of inflationary pressures.

13. The CBS will continue to expand its policy instruments by introducing a standing credit facility, using its portfolio of treasury bills for open market operations, and introducing repo and reverse repo transactions. Cash flow management was strengthened through improved cooperation between the Ministry of Finance and the CBS, and a good progress was achieved in developing liquidity forecasting and a reserve money programming framework.

14. Consistent with the price stability objective, the exchange regime will remain a market-based float to provide a buffer against changes in the external environment. The CBS interventions in the foreign exchange market will aim at limiting exceptional volatility of the rupee and ensuring orderly market conditions, subject to the achievement of the external reserves and reserve money targets.

\section{Overall, the banking sector appears stable and vulnerabilities manageable}

(Table 5). Despite severe interest and exchange rate shocks of late 2008, banks reported net profits and their capitalization improved. Although credit risks still remain, especially given the worsening external environment and declining tourism, stress tests suggest that the banking sector would withstand a fairly strong credit shock. ${ }^{7}$ Modernization of the regulatory framework and strengthening of the supervisory capacity are ongoing, drawing heavily on IMF TA recommendations. Privatization of state-owned banks remains the authorities' medium-term objective.

\section{External Sector, Financing, and Debt Sustainability}

16. The current account deficit is projected to shrink considerably in 2009 . The expected contraction of both tourism and fishing receipts, reflecting the slowdown in the global economy and rising piracy in Seychelles' territorial waters is likely to be more than offset by a sharp drop in imports - primarily as a result of falling international food and fuel prices. FDI, which has played an increasingly important role in developing the tourism sector, is expected to decline in 2009 as international investors scale back or postpone their investment projects, but recover gradually in subsequent years. Together with a return to normal import levels, this is likely to lead to a small deterioration in the external current account deficit in 2010, followed by an improvement to an average of 25 percent of GDP in 2011-14 (Table 6).

\footnotetext{
${ }^{7}$ Stress tests suggest that capital adequacy of only one bank would drop below the prudential norm of 12 percent, but would remain above the 8 percent Basel I ratio.
} 
17. There has been good progress made towards the debt restructuring objectives envisaged under the program. The authorities have been continuing good faith efforts to preserve inter-creditor equity in compliance with the Fund's lending into arrears policy, and developments in debtor-creditor relations are compatible with program implementation. The authorities completed a second road show for commercial and other creditors in March and maintained a dialogue with all creditors. Creditors' feedback on the proposals presented on the second road show will inform the final debt exchange offer. Payments to two external commercial bank creditors were made in recent months from escrow accounts not under the authorities' control. Instructions to stop payment into these accounts were issued and account balances are minimal. Payments to creditors will be accounted for in a debt exchange offer with a view to preserving inter-creditor equity.

18. An updated debt sustainability assessment (DSA), following the Paris Club agreement and under the updated program assumptions, shows that public debt remains highly unsustainable (Appendix II). Under a baseline scenario, with Paris Club and South African debt restructured along Paris Club agreement terms, and assuming that new borrowing on commercial terms is undertaken to refinance non-Paris Club arrears and close financing gaps, public external debt would reach 326 percent of GDP by 2019. Assuming that other creditors agree to restructure Seychelles' public external debt on terms comparable to those granted by the Paris Club, external financing gaps would be closed and the public external debt stock would decline to 63 percent of GDP by 2019. Bound tests show that Seychelles remains highly vulnerable to a large variety of shocks.

19. Debt management capacity of the Ministry of Finance has improved, but further progress is needed in line with technical assistance provided by the Fund, and the need to formulate a medium-term debt strategy. To this end, the authorities will seek further technical assistance from the Fund and other TA providers.

20. Financing gaps have increased somewhat in 2009-10, while the medium-term outlook remains broadly as envisaged at the time of the first program and financing assurances review (Table 7). Development lending operations by the World Bank and African Development Bank for 2009-10 (US\$38 million) are proceeding as expected, with US\$19 million available in mid-2009. Remaining financing gaps are expected to be closed by debt restructuring and by additional program support, primarily from the EU, in 2009-10.

\section{Program Design, Monitoring, AND Risks}

\section{A. Program Design and Monitoring}

\section{The revised quarterly quantitative PCs for June, September, and}

December 2009 are set out in the MEFP, Table 1. The revision to the quantitative PCs reflects the need to capture the progress in macro stabilization, but also the impact of global recession and heightened piracy on the program targets. The phasing of access has not been changed and Seychelles' should not have difficulty meeting its obligations to the Fund 
(Tables 8 and 9). New structural benchmarks have been added for the later part of 2009 to target strengthening operations at the CBS (including to address areas identified in the safeguards assessment), strengthening monitoring of parastatal performance, and implementing the tax reform (MEFP; Table 2).

\section{B. Risks}

22. The risks to the program have moderated significantly since it was put in place, but remain largely associated with a difficult external economic environment, implementation capacity constraints and the impact of negative growth on households. The risks to the program appear manageable and are balanced against the authorities' over performance relative to program objectives and rapid stabilization of the macroeconomic environment. Continued Fund technical assistance to the CBS and Ministry of Finance has been invaluable to program success thus far.

\section{Staff Appraisal}

23. The staff commends the authorities for the strong performance to date under the program. Rapid macroeconomic stabilization has led to a virtuous cycle whereby lower budget financing costs and stronger demand for money are aiding the adjustment effort. This positive environment was made possible through a significant fiscal adjustment effort, backed by the new market-based monetary policy. Staff encourages the authorities to build on this success by working to entrench prudent macroeconomic policies in support of their objective of restoring fiscal sustainability and laying the foundation for economic recovery.

\section{Staff welcomes the strengthening of public financial management.}

Implementation of the treasury single account currently in process is a key component of these reforms. The authorities are encouraged to maintain the pace of efforts to transform the management of public finance.

25. Progress is being made to reinforce financial discipline and accountability in the parastatal sector, which if left unaddressed, could put at risk the hard-won recent stabilization gains. Major improvements in public sector governance practices are needed over the medium-term to contain fiscal risks and raise productivity in the parastatal sector.

26. The authorities have strengthened tax administration and elaborated a fundamental tax policy reform to be implemented over the medium-term. This initiative is key to improving the business and investment climate, reducing economic distortions, and securing fiscal sustainability.

27. The new monetary policy framework has played a key role in the rapid economic stabilization. The managed floating exchange regime remains appropriate. Looking ahead, efforts should focus on further expanding monetary policy instruments and modernizing the legal and institutional framework for the financial sector. 
28. Staff encourages the authorities to continue to reinforce debt management capacity and to pursue their public external debt restructuring strategy aimed at normalizing relations with all creditors. Given their continuing good faith efforts to reach agreement on comparable terms with all creditors, as evidenced by the ongoing dialogue and timely sharing of information, the staff believes the authorities are in compliance with the Fund's lending into arrears policy. Continued Fund involvement is crucial to the success of the reform effort.

29. The staff believes that the policies in place are sufficient to achieve the program's objectives. While program financing needs for 2009-10 are now somewhat higher than previously expected, additional budget and balance of payment support appears to be forthcoming, and the program is financed, assuming a public debt restructuring consistent with Seychelles' payments capacity can be achieved.

30. In view of the reduced risks to the program reflecting the strong performance to date and rapid progress in macro stabilization staff recommends completion of the second program and financing assurances reviews. Staff supports the modification of the quarterly performance criteria. Staff proposes to shift to semi-annual program reviews, while maintaining quarterly performance criteria, and associated purchases, and financing assurances reviews. The authorities have indicated that, in support of their medium-term reform agenda, they intend to request support under the Fund's Extended Fund facility, in replacement of the SBA, by end-2009. 
Table 1. Seychelles: Selected Economic and Financial Indicators, 2006-10

\begin{tabular}{|c|c|c|c|c|c|c|c|}
\hline & \multirow[t]{2}{*}{2006} & \multirow[t]{2}{*}{2007} & \multicolumn{2}{|c|}{2008} & \multicolumn{2}{|c|}{2009} & \multirow{2}{*}{2010} \\
\hline & & & Prog. & Prel. & $\begin{array}{c}\text { Country } \\
\text { Report No. } \\
09 / 121\end{array}$ & Proj. & \\
\hline National income and prices ${ }^{1}$ & \multicolumn{7}{|c|}{ (Percentage change, unless otherwise indicated) } \\
\hline Nominal GDP (millions of Seychelles rupees) & 5,628 & 6,877 & 7,878 & 8,756 & 9,650 & 10,280 & 11,066 \\
\hline Real GDP & 9.3 & 9.7 & 3.1 & -0.9 & -9.6 & -10.7 & 3.5 \\
\hline $\mathrm{CPI}$ (annual average) & -1.9 & 5.3 & 32.2 & 37.0 & 39.2 & 35.1 & 4.1 \\
\hline CPI (end-of-period) & 0.5 & 16.8 & 38.8 & 63.3 & 16.3 & 5.4 & 2.2 \\
\hline GDP deflator average & 2.1 & 11.4 & 25.0 & 28.4 & 34.7 & 31.4 & 4.0 \\
\hline Money and credit & \multicolumn{7}{|c|}{ (Percentage change, unless otherwise indicated) } \\
\hline Net claims on private sector & 0.4 & 9.3 & 12.5 & 58.4 & 13.1 & 24.3 & 20.2 \\
\hline Broad money $(M 3(p))^{2}$ & 6.1 & -7.6 & 16.1 & 27.2 & 19.2 & 5.6 & 20.5 \\
\hline Reserve money & 32.7 & -23.1 & 3.4 & 0.6 & 20.6 & 20.6 & 19.4 \\
\hline Velocity (GDP/M3) & 1.1 & 1.5 & 1.5 & 1.5 & 1.5 & 1.6 & 1.5 \\
\hline Money multiplier (M3/reserve money) & 3.2 & 4.2 & 4.7 & 5.3 & 4.8 & 4.7 & 4.7 \\
\hline & \multicolumn{7}{|c|}{ (In percent of GDP) } \\
\hline External savings & 13.2 & 20.8 & 28.8 & 45.6 & 29.3 & 29.7 & 33.0 \\
\hline Gross national savings & 13.5 & 8.0 & 4.9 & -13.7 & 2.2 & -1.8 & -3.8 \\
\hline Of which: government savings & 0.4 & -4.4 & 3.1 & 4.2 & -2.2 & 1.0 & 4.5 \\
\hline Gross investment & 26.6 & 28.8 & 33.6 & 31.9 & 31.5 & 27.9 & 29.3 \\
\hline Of which: government investment & 7.7 & 5.5 & 4.1 & 2.1 & 3.7 & 4.3 & 5.0 \\
\hline Government budget & \multicolumn{7}{|c|}{ (In percent of GDP) } \\
\hline Total revenue, excluding grants & 39.9 & 32.0 & 35.4 & 32.8 & 35.1 & 35.1 & 34.1 \\
\hline Expenditure and net lending & 47.0 & 40.9 & 37.6 & 39.8 & 40.1 & 35.6 & 32.9 \\
\hline Current expenditure & 39.4 & 36.3 & 32.2 & 28.6 & 37.3 & 34.2 & 29.6 \\
\hline Capital expenditure and net lending & 7.6 & 4.6 & 5.4 & 11.2 & -0.3 & 0.5 & 2.4 \\
\hline Overall balance, including grants & -5.9 & -8.7 & -1.8 & -3.3 & -4.8 & 0.0 & 1.4 \\
\hline Primary balance ${ }^{3}$ & -0.5 & -2.0 & 7.1 & 3.8 & 9.8 & 11.4 & 9.8 \\
\hline Total public debt ${ }^{4}$ & 132.4 & 129.8 & 151.3 & 139.4 & 177.8 & 149.7 & 121.7 \\
\hline Domestic & 81.4 & 65.8 & 53.5 & 52.3 & 44.2 & 41.8 & 30.5 \\
\hline External $^{4}$ & 51.0 & 63.9 & 97.8 & 87.1 & 133.6 & 108.0 & 91.2 \\
\hline External sector & \multicolumn{7}{|c|}{ (In percent of GDP, unless otherwise indicated) } \\
\hline Current account balance including official transfers & -13.2 & -20.8 & -28.8 & -45.6 & -29.3 & -29.7 & -33.0 \\
\hline Total stock of arrears (millions of U.S. dollars) ${ }^{4}$ & 123.5 & 160.4 & 330.6 & 333.5 & $\ldots$ & 195.1 & $\ldots$ \\
\hline Total external debt outstanding (millions of U.S. dollars) ${ }^{4,5}$ & 521.0 & 710.2 & 840.5 & 802.2 & 805.6 & 732.6 & 694.7 \\
\hline Total external debt outstanding ${ }^{5}$ & 51.1 & 69.2 & 97.8 & 87.1 & 133.6 & 108.0 & 91.2 \\
\hline Terms of trade (= - deterioration) & -0.2 & -1.2 & 2.1 & 0.7 & -3.1 & -2.1 & $\ldots$ \\
\hline Real effective exchange rate (end-of-period, percent change) & -9.7 & -25.4 & -8.5 & -18.4 & $\cdots$ & $\ldots$ & .. \\
\hline 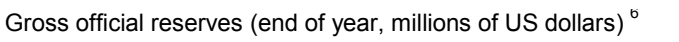 & 113 & 10 & 19 & 51 & 91 & 108 & 158 \\
\hline In months of imports, c.i.f. & 1.3 & 0.1 & 0.2 & 0.7 & 1.2 & 1.3 & 1.8 \\
\hline \multicolumn{8}{|l|}{ Exchange rate } \\
\hline Seychelles rupees per US\$1 (end of period) & 5.8 & 8.0 & 14.0 & 16.6 & 16.0 & $\ldots$ & ... \\
\hline Seychelles rupees per US\$1 (period average) & 5.5 & 6.7 & 9.0 & 9.5 & 16.0 & $\ldots$ & ... \\
\hline
\end{tabular}

Sources: Central Bank of Seychelles; Ministry of Finance; and IMF staff estimates and projections.

${ }^{1}$ Historic GDP data have been revised upwards in April 2009.

${ }^{2}$ In 2007 and earlier, includes domestic currency balances earmarked for pending import requests ("pipeline").

${ }^{3}$ The CBS transferred SR 175 million (2.2 percent of GDP) in profit to the government in March 2008. Of this amount SR 118.7 million (1.5 percent of GDP) was revaluation gains

${ }^{4}$ Assumes April 2009 Paris Club agreement is implemented.

${ }^{5}$ Includes arrears and the external debt of the central bank.

${ }^{6}$ Prior to 2007 includes blocked deposits and project accounts. 
Table 2. Seychelles: Consolidated Government Operations, 2007-10 ${ }^{1}$

(Millions of Seychelles rupees)

\begin{tabular}{|c|c|c|c|c|c|c|c|c|c|c|c|c|c|c|c|c|c|c|}
\hline & \multirow{2}{*}{\multicolumn{2}{|c|}{$2007 \quad 2008$}} & \multicolumn{15}{|c|}{2009} & \multirow{3}{*}{$\begin{array}{l}2010 \\
\text { Proj. }\end{array}$} \\
\hline & & & \multicolumn{3}{|c|}{ Q1 } & \multicolumn{3}{|c|}{ Q2 } & \multicolumn{3}{|c|}{ Q3 } & \multicolumn{3}{|c|}{ Q4 } & \multicolumn{3}{|c|}{ Year } & \\
\hline & Act. & Actual & Prog. & Proj. & Act. & Prog. & $\begin{array}{l}\text { Revised } \\
\text { Prog. }\end{array}$ & New. Proj. & Prog. & $\begin{array}{l}\text { Revised } \\
\text { Prog. }\end{array}$ & New. Proj. & Prog. & $\begin{array}{l}\text { Revised } \\
\text { Prog. }\end{array}$ & New. Proj. & Prog. & $\begin{array}{l}\text { Revised } \\
\text { Prog. }\end{array}$ & New. Proj. & \\
\hline Total revenue and grants & $2,214.2$ & $3,189.7$ & 826.2 & 917.0 & $1,057.8$ & 759.4 & 726.3 & 782.0 & 925.2 & 848.6 & 876.1 & $1,052.9$ & 918.6 & 941.0 & $3,563.6$ & $3,410.5$ & $3,656.8$ & $3,793.6$ \\
\hline Total revenue & $2,197.5$ & $2,868.5$ & 821.2 & 912.0 & $1,055.3$ & 754.4 & 721.3 & 765.4 & 920.2 & 843.6 & 854.2 & $1,047.9$ & 913.6 & 936.0 & $3,543.6$ & $3,390.5$ & $3,610.8$ & $3,773.6$ \\
\hline $\operatorname{Tax}$ & $1,896.4$ & $2,456.3$ & 695.8 & 779.2 & 907.1 & 702.8 & 622.8 & 646.6 & 848.8 & 711.6 & 756.1 & 886.0 & 740.9 & 755.9 & $3,133.5$ & $2,854.5$ & $3,065.7$ & $3,158.0$ \\
\hline Social security tax & 301.3 & 294.9 & 77.4 & 77.4 & 94.1 & 88.0 & 88.0 & 85.6 & 91.5 & 91.5 & 92.6 & 95.0 & 91.5 & 92.7 & 352.0 & 348.4 & 365.0 & 375.0 \\
\hline Trade tax & 265.2 & 409.6 & 115.0 & 90.7 & 103.5 & 132.3 & 104.3 & 118.0 & 155.3 & 122.5 & 118.0 & 172.5 & 136.1 & 127.0 & 575.0 & 453.6 & 466.5 & 500.2 \\
\hline Goods and services tax (GST) & 799.6 & 950.6 & 336.4 & 256.3 & 320.1 & 343.5 & 286.1 & 311.4 & 352.5 & 293.2 & 346.0 & 409.6 & 323.0 & 344.2 & $1,442.0$ & $1,158.6$ & $1,321.7$ & $1,350.9$ \\
\hline Business tax & 360.5 & 524.0 & 106.1 & 115.0 & 126.8 & 107.8 & 110.0 & 110.0 & 168.7 & 170.0 & 170.0 & 168.5 & 156.0 & 156.0 & 551.0 & 551.0 & 562.8 & 593.1 \\
\hline Other & 169.8 & 277.3 & 60.9 & 239.7 & 262.7 & 31.3 & 34.4 & 21.6 & 80.9 & 34.4 & 29.5 & 40.4 & 34.4 & 35.9 & 213.5 & 342.9 & 349.7 & 338.7 \\
\hline Nontax & 301.1 & 412.2 & 125.4 & 132.8 & 148.1 & 51.6 & 98.5 & 118.7 & 71.4 & 132.0 & 98.0 & 161.9 & 172.7 & 180.1 & 410.2 & 536.0 & 545.0 & 615.6 \\
\hline Fees and charges & 175.8 & 145.3 & 43.1 & 48.5 & 68.5 & 41.0 & 39.6 & 32.1 & 38.9 & 48.5 & 37.0 & 42.1 & 61.4 & 40.1 & 165.0 & 198.1 & 177.8 & 274.6 \\
\hline Dividends from parastatals & 94.8 & 64.1 & 0.0 & 0.0 & 5.0 & 0.0 & 0.0 & 42.2 & 16.0 & 16.0 & 14.0 & 107.0 & 94.0 & 89.0 & 123.0 & 110.0 & 150.2 & 110.0 \\
\hline Other & 30.5 & 202.7 & 82.3 & 84.3 & 74.6 & 10.6 & 58.9 & 44.4 & 16.4 & 67.4 & 47.0 & 12.8 & 17.3 & 51.0 & 122.2 & 227.9 & 217.0 & 231.1 \\
\hline External grants & 16.7 & 321.2 & 5.0 & 5.0 & 2.5 & 5.0 & 5.0 & 16.6 & 5.0 & 5.0 & 22.0 & 5.0 & 5.0 & 5.0 & 20.0 & 20.0 & 46.1 & 20.0 \\
\hline Expenditure and net lending & $2,810.2$ & $3,482.9$ & $1,000.6$ & 942.5 & 518.3 & $1,145.7$ & $1,085.8$ & 950.8 & 950.8 & 845.6 & 913.0 & $1,073.8$ & 999.0 & $1,129.4$ & $4,171.0$ & $3,872.8$ & $3,660.3$ & $3,638.7$ \\
\hline Current expenditure & $2,497.2$ & $2,503.5$ & 829.1 & 864.1 & 739.6 & 963.6 & 999.0 & $1,010.3$ & 804.7 & 797.7 & 794.2 & 917.2 & 942.6 & 967.2 & $3,514.6$ & $3,603.4$ & $3,511.3$ & $3,275.3$ \\
\hline Primary current expenditure & $2,041.4$ & $1,874.6$ & 581.7 & 546.6 & 494.5 & 561.7 & 544.8 & 619.1 & 568.3 & 545.8 & 609.4 & 573.5 & 558.3 & 612.2 & $2,285.2$ & $2,195.5$ & $2,335.2$ & $2,348.8$ \\
\hline Wages and salaries & 699.7 & 688.1 & 206.9 & 184.4 & 198.9 & 180.1 & 168.6 & 196.3 & 180.1 & 168.6 & 184.8 & 180.1 & 168.6 & 187.9 & 747.2 & 690.3 & 767.9 & 683.3 \\
\hline Goods and services & 499.8 & 488.8 & 146.7 & 153.8 & 118.0 & 151.9 & 160.8 & 169.6 & 161.2 & 160.8 & 184.7 & 165.3 & 172.2 & 188.5 & 625.0 & 647.6 & 660.9 & 747.2 \\
\hline Interest payments due & 455.8 & 628.9 & 247.4 & 317.6 & 245.1 & 402.0 & 454.2 & 391.2 & 236.4 & 251.8 & 184.8 & 343.7 & 384.3 & 355.0 & $1,229.4$ & $1,407.9$ & $1,176.1$ & 926.5 \\
\hline Foreign interest ${ }^{\circ}$ & 219.4 & 343.8 & 43.8 & 47.9 & 63.1 & 196.3 & 216.9 & 227.5 & 41.0 & 45.2 & 38.7 & 181.4 & 208.4 & 211.9 & 462.5 & 518.4 & 541.3 & 434.5 \\
\hline Domestic interest & 236.4 & 285.1 & 203.5 & 269.7 & 181.9 & 205.7 & 237.3 & 163.7 & 195.4 & 206.7 & 146.1 & 162.3 & 175.9 & 143.0 & 766.9 & 889.6 & 634.8 & 491.9 \\
\hline Transfers & 833.6 & 692.0 & 226.2 & 205.9 & 170.2 & 227.7 & 212.9 & 242.8 & 225.0 & 213.9 & 237.9 & 226.1 & 214.9 & 234.6 & 905.1 & 847.4 & 885.6 & 908.0 \\
\hline Social program of central government & 166.7 & 192.3 & 88.3 & 67.0 & 40.2 & 89.8 & 74.0 & 73.7 & 88.3 & 75.0 & 87.3 & 89.3 & 76.0 & 83.5 & 355.8 & 292.0 & 284.7 & 360.3 \\
\hline Transfers to public sector from central government & 375.6 & 220.5 & 45.6 & 46.5 & 47.0 & 45.6 & 46.5 & 79.9 & 44.4 & 46.5 & 60.1 & 44.5 & 46.5 & 58.8 & 180.1 & 186.1 & 245.8 & 150.2 \\
\hline Benefits and programs of Social Security Fund & 291.3 & 279.2 & 92.3 & 92.3 & 83.0 & 92.3 & 92.3 & 89.2 & 92.3 & 92.3 & 90.6 & 92.3 & 92.3 & 92.3 & 369.2 & 369.3 & 355.1 & 397.5 \\
\hline Other & 8.3 & 5.7 & 2.0 & 2.6 & 7.4 & 2.0 & 2.6 & 10.3 & 2.0 & 2.6 & 1.9 & 2.0 & 2.6 & 1.2 & 8.0 & 10.2 & 20.8 & 10.3 \\
\hline $\begin{array}{l}\text { Capital expenditure } \\
\text { Externally financed }\end{array}$ & 375.7 & 183.2 & 71.5 & 74.3 & 21.3 & 82.1 & 82.7 & 111.8 & 96.1 & 94.0 & 136.3 & 106.7 & 102.4 & 176.7 & 356.4 & 353.4 & 446.1 & 555.4 \\
\hline $\begin{array}{l}\text { Net lending } \\
\text { Contingency }\end{array}$ & -62.7 & 796.2 & $\begin{array}{r}0.0 \\
100.0\end{array}$ & $\begin{array}{l}-96.0 \\
100.0\end{array}$ & $\begin{array}{r}-242.5 \\
0.0\end{array}$ & $\begin{array}{r}0.0 \\
100.0\end{array}$ & $\begin{array}{l}-96.0 \\
100.0\end{array}$ & $\begin{array}{r}-22.5 \\
0.0\end{array}$ & $\begin{array}{r}0.0 \\
50.0\end{array}$ & $\begin{array}{r}-96.0 \\
50.0\end{array}$ & $\begin{array}{r}-67.5 \\
50.0\end{array}$ & $\begin{array}{r}0.0 \\
50.0\end{array}$ & $\begin{array}{r}-96.0 \\
50.0\end{array}$ & $\begin{array}{r}-64.5 \\
50.0\end{array}$ & $\begin{array}{r}0.0 \\
300.0\end{array}$ & $\begin{array}{r}-384.0 \\
300.0\end{array}$ & $\begin{array}{r}-397.0 \\
100.0\end{array}$ & $\begin{array}{r}-292.0 \\
100.0\end{array}$ \\
\hline Primary balance $^{<}$ & -140.2 & 335.7 & 72.9 & 292.2 & 784.5 & 15.7 & 94.7 & 73.5 & 210.8 & 254.8 & 148.0 & 322.8 & 304.0 & 166.6 & 622.0 & 945.6 & $1,172.6$ & $1,081.4$ \\
\hline Overall balance, commitment basis & -596.0 & -293.2 & -174.4 & -25.4 & 539.4 & -386.3 & -359.5 & -317.7 & -25.6 & 2.9 & -36.8 & -20.9 & -80.4 & -188.4 & -607.4 & -462.3 & -3.5 & 154.9 \\
\hline Change in arrears & 59.4 & 31.2 & 0.0 & -61.5 & -14.1 & 0.0 & -50.0 & -50.0 & -181.4 & -50.0 & -50.0 & 0.0 & -50.0 & -34.0 & -181.4 & -211.5 & -148.1 & 0.0 \\
\hline External interest & 3.9 & 166.8 & 0.0 & 0.0 & 46.8 & 0.0 & 0.0 & 0.0 & 0.0 & 0.0 & 0.0 & 0.0 & 0.0 & 0.0 & 0.0 & 0.0 & 46.8 & 0.0 \\
\hline Budget & 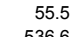 & -135.6 & 0.0 & -61.5 & $\begin{array}{r}-60.9 \\
5525\end{array}$ & 0.0 & -50.0 & -50.0 & -181.4 & -50.0 & -50.0 & 0.0 & -50.0 & -34.0 & -181.4 & -211.5 & -194.9 & $\begin{array}{r}0.0 \\
15.9\end{array}$ \\
\hline Overall balance, cash basis (after grants) & -536.6 & -262.0 & -174.4 & -87.0 & 525.3 & -386.3 & -409.5 & -367.7 & -207.0 & -47.1 & -86.8 & -20.9 & -130.4 & -222.4 & -788.8 & -673.9 & -151.6 & 154.9 \\
\hline Financing & 536.6 & 262.0 & 174.4 & 87.0 & -525.3 & 386.3 & 409.5 & 367.7 & -7.6 & -126.8 & 86.8 & -296.3 & -278.1 & 222.4 & 788.8 & 673.9 & 151.6 & -154.9 \\
\hline Foreign financing & 410.8 & 232.8 & -150.7 & -171.1 & -258.6 & -450.0 & -515.5 & -546.3 & 64.4 & 139.1 & 127.4 & -178.1 & -190.8 & -174.7 & -714.4 & -738.2 & -852.3 & -316.4 \\
\hline Disbursements & 859.4 & 671.8 & 70.0 & 80.0 & 2.2 & 70.0 & 80.0 & 0.0 & 280.0 & 384.0 & 351.6 & 70.0 & 80.0 & 73.3 & 490.0 & 624.0 & 427.1 & 566.3 \\
\hline Project loans & 859.4 & 671.8 & 70.0 & 80.0 & 2.2 & 70.0 & 80.0 & 0.0 & 70.0 & 80.0 & 73.3 & 70.0 & 80.0 & 73.3 & 280.0 & 320.0 & 148.7 & 290.4 \\
\hline Program/budget support & 0.0 & 0.0 & 0.0 & 0.0 & 0.0 & 0.0 & $\begin{array}{r}0.0 \\
5055\end{array}$ & 0.0 & 210.0 & 304.0 & 278.4 & 0.0 & 0.0 & 0.0 & 210.0 & 304.0 & 278.4 & 275.9 \\
\hline Scheduled amortization & -448.6 & $-1,629.8$ & -220.7 & -251.1 & -260.9 & -520.0 & -595.5 & -546.3 & -215.6 & -244.9 & -224.2 & -248.1 & -270.8 & -248.0 & $-1,204.4$ & $-1,362.2$ & $-1,279.4$ & -882.6 \\
\hline Change in amortization arrears & -2.7 & $1,190.8$ & 0.0 & 0.0 & 228.3 & 0.0 & 0.0 & $-2,055.1$ & 0.0 & 0.0 & 0.0 & 0.0 & 0.0 & 0.0 & 0.0 & 0.0 & $-1,826.8$ & 0.0 \\
\hline Debt relief & 0.0 & 0.0 & 0.0 & 0.0 & 0.0 & 0.0 & 0.0 & 2055.1 & 0.0 & 0.0 & 0.0 & 0.0 & 0.0 & 0.0 & 0.0 & 0.0 & 2055.1 & 0.0 \\
\hline Domestic financing, net & -19.5 & -69.8 & 108.1 & 79.0 & -394.6 & 276.5 & 145.8 & 193.4 & -174.0 & -332.7 & -379.4 & -289.0 & -257.1 & 4.2 & -78.4 & -404.9 & -576.4 & -913.6 \\
\hline Bank financing & -50.3 & -99.6 & 102.7 & 75.1 & $-1,335.6$ & 262.6 & 138.5 & 183.7 & -165.3 & -306.6 & -360.4 & -274.5 & -244.2 & 4.0 & -74.5 & -384.7 & $-1,508.3$ & -867.9 \\
\hline CBS & 27.9 & -3.4 & 10.8 & 23.7 & -948.4 & 27.6 & 43.7 & 19.3 & -17.4 & -99.8 & -37.9 & -28.9 & -77.1 & 0.4 & -7.8 & -121.5 & -966.5 & -91.4 \\
\hline Commercial banks & -118.9 & -96.2 & 91.9 & 51.4 & -387.2 & 235.0 & 94.8 & 164.4 & -147.9 & -216.3 & -322.5 & -245.6 & -167.1 & 3.6 & -66.7 & -263.2 & -541.7 & -776.5 \\
\hline Nonbank & 30.7 & 29.8 & 5.4 & 4.0 & 941.0 & 13.8 & 7.3 & 9.7 & -8.7 & -16.6 & -19.0 & -14.4 & -12.9 & 0.2 & -3.9 & -20.2 & 931.9 & -45.7 \\
\hline Privatization and long-term lease of fixed assets & 134.3 & 219.4 & 85.6 & 56.8 & 96.6 & 36.0 & 56.8 & 93.0 & 102.0 & 66.8 & 90.0 & 170.8 & 169.8 & 90.0 & 394.3 & 390.2 & 369.7 & 57.6 \\
\hline $\begin{array}{l}\text { Statistical discrepancy } \\
\text { Fiscal financing gap }\end{array}$ & $\begin{array}{r}13.8 \\
0.0\end{array}$ & $\begin{array}{r}-120.4 \\
0.0\end{array}$ & $\begin{array}{r}0.0 \\
131.5\end{array}$ & $\begin{array}{r}0.0 \\
122.1\end{array}$ & $\begin{array}{r}-197.0 \\
0.0\end{array}$ & $\begin{array}{r}0.0 \\
523.9\end{array}$ & $\begin{array}{r}0.0 \\
722.3\end{array}$ & $\begin{array}{r}0.0 \\
6276\end{array}$ & $\begin{array}{r}0.0 \\
2146\end{array}$ & $\begin{array}{r}0.0 \\
1739\end{array}$ & $\begin{array}{r}0.0 \\
2488\end{array}$ & $\begin{array}{r}0.0 \\
3172\end{array}$ & $\begin{array}{r}0.0 \\
408.5\end{array}$ & $\begin{array}{r}0.0 \\
3029\end{array}$ & $\begin{array}{r}0.0 \\
11872\end{array}$ & $\begin{array}{r}0.0 \\
1.4268\end{array}$ & $\begin{array}{r}-197.0 \\
1.1793\end{array}$ & $\begin{array}{r}0.0 \\
1.0174\end{array}$ \\
\hline
\end{tabular}


Table 2. Seychelles: Consolidated Government Operations, 2007-10 ${ }^{1}$ (continued)

(Percent of GDP)

\begin{tabular}{|c|c|c|c|c|c|c|c|c|c|c|c|c|c|c|c|c|c|c|}
\hline & \multirow{3}{*}{$\begin{array}{r}2007 \\
\text { Act. }\end{array}$} & \multirow{3}{*}{$\begin{array}{c}2008 \\
\text { Actual }\end{array}$} & \multicolumn{15}{|c|}{2009} & \multirow{3}{*}{$\begin{array}{l}2010 \\
\text { Proj. }\end{array}$} \\
\hline & & & \multicolumn{3}{|c|}{ Q1 } & \multicolumn{3}{|c|}{ Q2 } & \multicolumn{3}{|c|}{ Q3 } & \multicolumn{3}{|c|}{ Q4 } & \multicolumn{3}{|c|}{ Year } & \\
\hline & & & Prog. & Proj. & Act. & Prog. & $\begin{array}{l}\text { Revised } \\
\text { Prog. }\end{array}$ & New. Proj. & Prog. & $\begin{array}{l}\text { Revised } \\
\text { Prog. }\end{array}$ & New. Proj. & Prog. & $\begin{array}{l}\text { Revised } \\
\text { Prog. }\end{array}$ & New. Proj. & Prog. & $\begin{array}{l}\text { Revised } \\
\text { Prog. }\end{array}$ & New. Proj. & \\
\hline $\begin{array}{l}\text { Total revenue and grants } \\
\text { Total revenene. }\end{array}$ & 32.2 & 36.4 & 8.3 & 9.5 & 11.0 & 7.6 & 7.5 & 7.6 & 9.3 & 8.8 & 8.5 & 10.6 & 9.5 & 9.2 & 35.7 & 35.3 & 35.6 & 34.3 \\
\hline $\begin{array}{l}\text { Total revenue } \\
\text { Tax }\end{array}$ & 32.0 & 32.8 & 8.2 & 9.5 & 10.9 & 7.6 & 7.5 & 7.4 & 9.2 & 8.7 & 8.3 & 10.5 & 9.5 & 9.1 & 35.5 & 35.1 & 35.1 & 34.1 \\
\hline $\begin{array}{l}\text { Tax } \\
\text { Social security tax }\end{array}$ & 27.6 & 28.1 & 7.0 & 8.1 & 9.4 & 7.0 & 6.5 & 6.3 & 8.5 & 7.4 & 7.4 & 8.9 & 7.7 & 7.4 & 31.4 & 29.6 & 29.8 & 28.5 \\
\hline $\begin{array}{l}\text { Social security tax } \\
\text { Trade tax }\end{array}$ & $\begin{array}{l}4.4 \\
3.9\end{array}$ & 3.4 & 0.8 & 0.8 & 1.0 & 0.9 & 0.9 & 0.8 & 0.9 & 0.9 & 0.9 & 1.0 & 0.9 & 0.9 & 3.5 & 3.6 & 3.6 & 3.4 \\
\hline $\begin{array}{l}\text { Trade tax } \\
\text { Goods and services tax (GST) }\end{array}$ & 3.9 & 4.7 & 1.2 & 0.9 & 1.1 & 1.3 & 1.1 & 1.1 & 1.6 & 1.3 & 1.1 & 1.7 & 1.4 & 1.2 & 5.8 & 4.7 & 4.5 & 4.5 \\
\hline $\begin{array}{l}\text { Goods and services tax (GST) } \\
\text { Business tax }\end{array}$ & $\begin{array}{l}11.6 \\
52\end{array}$ & 10.9 & 3.4 & 2.7 & 3.3 & 3.4 & 3.0 & 3.0 & 3.5 & 3.0 & 3.4 & 4.1 & 3.3 & 3.3 & 14.5 & 12.0 & 12.9 & 12.2 \\
\hline $\begin{array}{l}\text { Business tax } \\
\text { Other }\end{array}$ & $\begin{array}{l}5.2 \\
2.5\end{array}$ & $\begin{array}{l}6.0 \\
3.2\end{array}$ & $\begin{array}{l}1.1 \\
0.6\end{array}$ & $\begin{array}{l}1.2 \\
2.5\end{array}$ & $\begin{array}{l}1.3 \\
2.7\end{array}$ & $\begin{array}{l}1.1 \\
0.3\end{array}$ & $\begin{array}{l}1.1 \\
0.4\end{array}$ & $\begin{array}{l}1.1 \\
02\end{array}$ & $\begin{array}{l}1.7 \\
0.8\end{array}$ & $\begin{array}{l}1.8 \\
0.4\end{array}$ & 1.7 & $\begin{array}{l}1.7 \\
0.4\end{array}$ & $\begin{array}{l}1.6 \\
0.4\end{array}$ & $\begin{array}{l}1.5 \\
0.3\end{array}$ & $\begin{array}{r}5.5 \\
21\end{array}$ & $\begin{array}{l}5.7 \\
36\end{array}$ & $\begin{array}{r}5.5 \\
3.4\end{array}$ & $\begin{array}{l}5.4 \\
3.1\end{array}$ \\
\hline $\begin{array}{l}\text { Other } \\
\text { Nontax }\end{array}$ & $\begin{array}{l}2.5 \\
4.4\end{array}$ & $\begin{array}{l}3.2 \\
4.7\end{array}$ & $\begin{array}{l}0.6 \\
1.3\end{array}$ & $\begin{array}{l}2.5 \\
1.4\end{array}$ & $\begin{array}{l}2.7 \\
1.5\end{array}$ & $\begin{array}{l}0.3 \\
0.5\end{array}$ & $\begin{array}{l}0.4 \\
1.0\end{array}$ & $\begin{array}{l}0.2 \\
1.2\end{array}$ & $\begin{array}{l}0.8 \\
0.7\end{array}$ & $\begin{array}{l}0.4 \\
1.4\end{array}$ & $\begin{array}{l}0.3 \\
1.0\end{array}$ & $\begin{array}{l}0.4 \\
1.6\end{array}$ & $\begin{array}{l}0.4 \\
1.8\end{array}$ & $\begin{array}{l}0.3 \\
1.8\end{array}$ & $\begin{array}{l}2.1 \\
4.1\end{array}$ & 3.6 & $\begin{array}{l}3.4 \\
53\end{array}$ & $\begin{array}{l}3.1 \\
5.6\end{array}$ \\
\hline Fees and charges & 2.6 & 1.7 & 0.4 & 0.5 & 0.7 & 0.4 & 0.4 & 0.3 & 0.4 & $\begin{array}{l}.4 \\
0.5\end{array}$ & 0.4 & $\begin{array}{l}1.6 \\
0.4\end{array}$ & $\begin{array}{l}1.8 \\
0.6\end{array}$ & $\begin{array}{l}1.0 \\
0.4\end{array}$ & $\begin{array}{l}4.1 \\
1.6\end{array}$ & $\begin{array}{l}5.6 \\
2.1\end{array}$ & $\begin{array}{l}5.3 \\
1.7\end{array}$ & $\begin{array}{l}5.6 \\
2.5\end{array}$ \\
\hline Dividends from parastatals & 1.4 & 0.7 & 0.0 & 0.0 & 0.1 & 0.0 & 0.0 & 0.4 & 0.2 & 0.2 & 0.1 & 1.1 & 1.0 & 0.9 & 1.2 & 1.1 & 1.5 & 1.0 \\
\hline Other' ${ }^{2}$ & 0.4 & 2.3 & 0.8 & 0.9 & 0.8 & 0.1 & 0.6 & 0.4 & 0.2 & 0.7 & 0.5 & 0.1 & 0.2 & 0.5 & 1.2 & 2.4 & 2.1 & 2.1 \\
\hline External grants & 0.2 & 3.7 & 0.1 & 0.1 & 0.0 & 0.1 & 0.1 & 0.2 & 0.1 & 0.1 & 0.2 & 0.1 & 0.1 & 0.0 & 0.2 & 0.2 & 0.2 & 0.2 \\
\hline Expenditure and net lending & 40.9 & 39.8 & 10.0 & 9.8 & 5.4 & 11.5 & 11.3 & 9.2 & 9.5 & 8.8 & 8.9 & 10.8 & 10.4 & 11.0 & 41.8 & 40.1 & 35.6 & 32.9 \\
\hline Current expenditure & 36.3 & 28.6 & 8.3 & 9.0 & 7.7 & 9.7 & 10.4 & 9.8 & 8.1 & 8.3 & 7.7 & 9.2 & 9.8 & 9.4 & 35.3 & 37.3 & 34.2 & 29.6 \\
\hline Primary current expenditure & 29.7 & 21.4 & 5.8 & 5.7 & 5.1 & 5.6 & 5.6 & 6.0 & 5.7 & 5.7 & 5.9 & 5.8 & 5.8 & 6.0 & 22.9 & 22.8 & 22.7 & 21.2 \\
\hline Wages and salaries & 10.2 & 7.9 & 2.1 & 1.9 & 2.1 & 1.8 & 1.7 & 1.9 & 1.8 & 1.7 & 1.8 & 1.8 & 1.7 & 1.8 & 7.5 & 7.2 & 7.5 & 6.2 \\
\hline Goods and services & 7.3 & 5.6 & 1.5 & 1.6 & 1.2 & 1.5 & 1.7 & 1.7 & 1.6 & 1.7 & 1.8 & 1.7 & 1.8 & 1.8 & 6.3 & 6.7 & 6.4 & 6.8 \\
\hline Interest payments due & 6.6 & 7.2 & 2.5 & 3.3 & 2.5 & 4.0 & 4.7 & 3.8 & 2.4 & 2.6 & 1.8 & 3.4 & 4.0 & 3.5 & 12.3 & 14.6 & 11.4 & 8.4 \\
\hline Foreign interest & 3.2 & 3.9 & 0.4 & 0.5 & 0.7 & 2.0 & 2.2 & 2.2 & 0.4 & 0.5 & 0.4 & 1.8 & 2.2 & 2.1 & 4.6 & 5.4 & 5.3 & 3.9 \\
\hline Domestic interest & 3.4 & 3.3 & 2.0 & 2.8 & 1.9 & 2.1 & 2.5 & 1.6 & 2.0 & 2.1 & 1.4 & 1.6 & 1.8 & 1.4 & 7.7 & 9.2 & 6.2 & 4.4 \\
\hline Transfers & 12.1 & 7.9 & 2.3 & 2.1 & 1.8 & 2.3 & 2.2 & 2.4 & 2.3 & 2.2 & 2.3 & 2.3 & 2.2 & 2.3 & 9.1 & 8.8 & 8.6 & 8.2 \\
\hline Social program of central government & 2.4 & 2.2 & 0.9 & 0.7 & 0.4 & 0.9 & 0.8 & 0.7 & 0.9 & 0.8 & 0.8 & 0.9 & 0.8 & 0.8 & 3.6 & 3.0 & 2.8 & 3.3 \\
\hline Transfers to public sector from central government & 5.5 & 2.5 & 0.5 & 0.5 & 0.5 & 0.5 & 0.5 & 0.8 & 0.4 & 0.5 & 0.6 & 0.4 & 0.5 & 0.6 & 1.8 & 1.9 & 2.4 & 1.4 \\
\hline $\begin{array}{l}\text { Benefits and programs of Social Security Fund } \\
\text { Other }\end{array}$ & 4.2 & 3.2 & 0.9 & 1.0 & 0.9 & 0.9 & 1.0 & 0.9 & 0.9 & 1.0 & 0.9 & 0.9 & 1.0 & 0.9 & 3.7 & 3.8 & 3.5 & 3.6 \\
\hline $\begin{array}{l}\text { Other } \\
\text { Capital expenditure }\end{array}$ & 0.1 & 0.1 & 0.0 & 0.0 & 0.1 & 0.0 & 0.0 & 0.1 & 0.0 & 0.0 & 0.0 & 0.0 & 0.0 & 0.0 & 0.1 & 0.1 & 0.2 & 0.1 \\
\hline $\begin{array}{l}\text { Capital expenditure } \\
\text { Net lending }\end{array}$ & 5.5 & 2.1 & 0.7 & 0.8 & 0.2 & 0.8 & 0.9 & 1.1 & 1.0 & 1.0 & 1.3 & 1.1 & 1.1 & 1.7 & 3.6 & 3.7 & 4.3 & 5.0 \\
\hline $\begin{array}{l}\text { Net lending } \\
\text { Contingency }\end{array}$ & -0.9 & 9.1 & 0.0 & -1.0 & -2.5 & 0.0 & -1.0 & -0.2 & 0.0 & -1.0 & -0.7 & 0.0 & -1.0 & -0.6 & 0.0 & -4.0 & -3.9 & -2.6 \\
\hline Primary balance & -2.0 & 3.8 & 0.7 & $\begin{array}{l}1.0 \\
3.0\end{array}$ & $\begin{array}{l}0.0 \\
8.1\end{array}$ & $\begin{array}{l}1.0 \\
0.2\end{array}$ & $\begin{array}{l}1.0 \\
1.0\end{array}$ & $\begin{array}{l}0.0 \\
0.7\end{array}$ & $\begin{array}{l}0.5 \\
2.1\end{array}$ & $\begin{array}{l}0.5 \\
2.6\end{array}$ & $\begin{array}{l}0.5 \\
1.4\end{array}$ & $\begin{array}{l}0.5 \\
3.2\end{array}$ & $\begin{array}{l}0.5 \\
3.1\end{array}$ & $\begin{array}{l}0.5 \\
1.6\end{array}$ & $\begin{array}{l}3.0 \\
6.2\end{array}$ & $\begin{array}{l}3.1 \\
9.8\end{array}$ & $\begin{array}{r}1.0 \\
114\end{array}$ & 0.9 \\
\hline Overall balance, commitment & -8.7 & -3.3 & -1.7 & -0.3 & 5.6 & -3.9 & $\begin{array}{l}-3.0 \\
\end{array}$ & $\begin{array}{c}-3.1 \\
-3.1\end{array}$ & -0.3 & 0.0 & $\begin{array}{l}1.4 \\
-0.4\end{array}$ & $\begin{array}{r}3.2 \\
-0.2\end{array}$ & $\begin{array}{l}3.1 \\
-0.8\end{array}$ & -1.8 & $\begin{array}{l}0.2 \\
-6.1\end{array}$ & $\begin{array}{l}9.8 \\
-4.8\end{array}$ & $\begin{array}{r}17.4 \\
0.0\end{array}$ & 1.4 \\
\hline Change in arrears & 0.9 & 0.4 & 0.0 & -0.6 & -0.1 & 0.0 & -0.5 & -0.5 & -1.8 & -0.5 & -0.5 & 0.0 & -0.5 & -0.3 & -1.8 & -2.2 & -1.4 & 0.0 \\
\hline External interest & 0.1 & 1.9 & 0.0 & 0.0 & 0.5 & 0.0 & 0.0 & 0.0 & 0.0 & 0.0 & 0.0 & 0.0 & 0.0 & 0.0 & 0.0 & 0.0 & 0.5 & 0.0 \\
\hline Budget & 0.8 & -1.5 & 0.0 & -0.6 & -0.6 & 0.0 & -0.5 & -0.5 & -1.8 & -0.5 & -0.5 & 0.0 & -0.5 & -0.3 & -1.8 & -2.2 & -1.9 & 0.0 \\
\hline Overall balance, cash basis (after grants) & -7.8 & -3.0 & -1.7 & -0.9 & 5.4 & -3.9 & -4.2 & -3.6 & -2.1 & -0.5 & -0.8 & -0.2 & -1.4 & -2.2 & -7.9 & -7.0 & -1.5 & 1.4 \\
\hline Financing & 7.8 & 3.0 & 1.7 & 0.9 & -5.4 & 3.9 & 4.2 & 3.6 & -0.1 & -1.3 & 0.8 & -3.0 & -2.9 & 2.2 & 7.9 & 7.0 & 1.5 & -1.4 \\
\hline Foreign financing & 5.9 & -10.9 & -1.5 & -1.8 & 0.0 & -4.5 & -5.3 & -25.3 & 0.6 & 1.4 & 1.2 & -1.8 & -2.0 & -1.7 & 0.0 & 0.0 & 0.0 & 0.0 \\
\hline Disbursements & 12.5 & 7.7 & 0.7 & 0.8 & 0.0 & 0.7 & 0.8 & 0.0 & 2.8 & 4.0 & 3.4 & 0.7 & 0.8 & 0.7 & 4.9 & 6.5 & 4.2 & 5.1 \\
\hline Project loans & 12.5 & 7.7 & 0.7 & 0.8 & 0.0 & 0.7 & 0.8 & 0.0 & 0.7 & 0.8 & 0.7 & 0.7 & 0.8 & 0.7 & 2.8 & 3.3 & 1.4 & 2.6 \\
\hline Program/budget support & 0.0 & 0.0 & 0.0 & 0.0 & 0.0 & 0.0 & 0.0 & 0.0 & 2.1 & 3.2 & 2.7 & 0.0 & 0.0 & 0.0 & 2.1 & 3.2 & 2.7 & 2.5 \\
\hline Scheduled amortization & -6.5 & -18.6 & -2.2 & -2.6 & -2.7 & -5.2 & -6.2 & -5.3 & -2.2 & -2.5 & -2.2 & -2.5 & -2.8 & -2.4 & -12.1 & -14.1 & -12.4 & -8.0 \\
\hline $\begin{array}{l}\text { Change in amortization arrears } \\
\text { Debtrelief }\end{array}$ & 0.0 & 13.6 & 0.0 & 0.0 & 2.4 & 0.0 & 0.0 & -20.0 & 0.0 & 0.0 & 0.0 & 0.0 & 0.0 & 0.0 & 0.0 & 0.0 & -17.8 & 0.0 \\
\hline $\begin{array}{l}\text { Debt relief } \\
\text { Domestic financing, net }\end{array}$ & 0.0 & $\begin{array}{r}0.0 \\
-0.8\end{array}$ & & & & & & & & & & & & & 0.0 & 0.0 & 20.0 & 0.0 \\
\hline $\begin{array}{l}\text { Domestic financing, net } \\
\text { Bank financing }\end{array}$ & -0.3 & -0.8 & 1.1 & 0.8 & -4.0 & 2.8 & 1.5 & 1.9 & -1.7 & -3.4 & -3.7 & -2.9 & -2.7 & 0.0 & -0.8 & -3.7 & -5.6 & -8.3 \\
\hline $\begin{array}{l}\text { Bann financiing } \\
\text { Nonbank }\end{array}$ & -0.7 & -1.1 & 1.0 & 0.8 & -13.4 & 2.6 & 1.4 & 1.8 & -1.7 & -3.1 & -3.6 & -2.8 & -2.4 & 0.0 & -0.7 & -3.5 & -14.7 & -7.8 \\
\hline $\begin{array}{l}\text { Nonbank } \\
\text { Privatization and long-term lease of fixed assets }\end{array}$ & $\begin{array}{l}0.4 \\
2.0\end{array}$ & $\begin{array}{l}0.3 \\
2.5\end{array}$ & $\begin{array}{l}0.1 \\
0.9\end{array}$ & $\begin{array}{l}0.0 \\
0.6\end{array}$ & $\begin{array}{l}9.4 \\
1.0\end{array}$ & $\begin{array}{l}0.1 \\
0.4\end{array}$ & $\begin{array}{l}0.1 \\
0.6\end{array}$ & $\begin{array}{l}0.1 \\
0.9\end{array}$ & $\begin{array}{c}-0.1 \\
1.0\end{array}$ & $\begin{array}{c}-0.2 \\
0.7\end{array}$ & $\begin{array}{r}-0.2 \\
0.9\end{array}$ & $\begin{array}{r}-0.1 \\
1.7\end{array}$ & $\begin{array}{c}-0.1 \\
1.8\end{array}$ & $\begin{array}{l}0.0 \\
0.9\end{array}$ & $\begin{array}{l}0.0 \\
4.0\end{array}$ & $\begin{array}{r}-0.2 \\
3.5\end{array}$ & $\begin{array}{l}9.1 \\
3.6\end{array}$ & $\begin{aligned}-0.4 \\
0.5\end{aligned}$ \\
\hline Statistical discrepancy & 0.2 & -1.4 & 0.0 & 0.0 & -2.0 & 0.0 & 0.0 & 0.0 & 0.0 & 0.0 & 0.0 & 0.0 & 0.0 & 0.0 & 0.0 & 0.0 & -1.9 & 0.0 \\
\hline Fiscal financing gap & 0.0 & 0.0 & 1.3 & 1.3 & 0.0 & 5.3 & 7.5 & 6.1 & 2.2 & 1.8 & 2.4 & 3.2 & 4.2 & 2.9 & 11.9 & 14.8 & 11.5 & 9.2 \\
\hline \multirow{2}{*}{$\begin{array}{l}\text { Memorandum item: } \\
\text { Nominal GDP }\end{array}$} & & & & & & & & & & & & & & & & & & \\
\hline & $6,877.0$ & $8,755.7$ & $9,970.1$ & $9,649.9$ & $9,649.9$ & 9,970.1 & $9,649.9$ & $10,280.4$ & 9,970.1 & $9,649.9$ & $10,280.4$ & $9,970.1$ & $9,649.9$ & $10,280.4$ & 9,970.1 & $9,649.9$ & $10,280.4$ & $11,066.4$ \\
\hline
\end{tabular}


Table 3. Seychelles: Monetary Survey and Central Bank Accounts, 2007-09

\begin{tabular}{|c|c|c|c|c|c|c|c|c|c|c|c|c|c|c|}
\hline & \multirow{3}{*}{$\begin{array}{l}2007 \\
\text { Dec. } \\
\text { Act. }\end{array}$} & \multicolumn{5}{|c|}{2008} & \multicolumn{8}{|c|}{2009} \\
\hline & & \multirow{2}{*}{$\begin{array}{l}\text { Mar. } \\
\text { Act. }\end{array}$} & \multirow{2}{*}{$\begin{array}{l}\text { Jun. } \\
\text { Act. }\end{array}$} & \multirow{2}{*}{$\begin{array}{l}\text { Sep. } \\
\text { Act. }\end{array}$} & \multicolumn{2}{|c|}{ Dec. } & \multicolumn{2}{|c|}{ Mar. } & \multicolumn{2}{|c|}{ Jun. } & \multicolumn{2}{|c|}{ Sep. } & \multicolumn{2}{|c|}{ Dec. } \\
\hline & & & & & Prog. & Act. & Prog. & Act. & Rev. prog. & Proj & Rev. prog. & Proj & Rev. prog. & Proj \\
\hline & \multicolumn{14}{|c|}{ (Seychelles rupees millions, unless otherwise indicated) } \\
\hline \multicolumn{15}{|l|}{ Monetary survey } \\
\hline Net foreign assets & 423 & 423 & 479 & 731 & 1,738 & 1,935 & 1,822 & 2,263 & 2,054 & 2,183 & 2,289 & 2,572 & 2,603 & 2,728 \\
\hline Central bank & 323 & 406 & 320 & 776 & 1,320 & 1,050 & 1,334 & 1,447 & 1,179 & 1,374 & 1,317 & 1,688 & 1,584 & 1,843 \\
\hline Deposit money banks & 101 & 17 & 160 & -45 & 418 & 885 & 488 & 816 & 876 & 809 & 972 & 884 & 1,020 & 885 \\
\hline Net domestic assets & 4,257 & 4,293 & 4,395 & 4,098 & 3,695 & 4,018 & 3,748 & 3,029 & 3,977 & 3,236 & 4,047 & 3,330 & 4,492 & 3,560 \\
\hline Domestic credit & 5,108 & 5,158 & 5,351 & 5,468 & 5,358 & 6,136 & 5,414 & 4,756 & 5,558 & 4,974 & 5,648 & 4,963 & 6,113 & 5,372 \\
\hline Net claims on the government & 3,178 & 3,122 & 3,279 & 3,240 & 2,928 & 3,079 & 3,031 & 1,743 & 3,063 & 1,927 & 2,747 & 1,566 & 2,503 & 1,570 \\
\hline Credit to the economy & 1,930 & 2,036 & 2,072 & 2,227 & 2,430 & 3,058 & 2,383 & 3,013 & 2,495 & 3,047 & 2,901 & 3,397 & 3,610 & 3,802 \\
\hline Other items, net & -851 & -865 & -957 & $-1,369$ & $-1,663$ & $-2,119$ & $-1,665$ & $-1,727$ & $-1,581$ & $-1,738$ & $-1,601$ & $-1,633$ & $-1,621$ & $-1,812$ \\
\hline M3 & 4,681 & 4,716 & 4,874 & 4,830 & 5,433 & 5,952 & 5,570 & 5,292 & 6,031 & 5,419 & 6,336 & 5,902 & 7,096 & 6,288 \\
\hline Foreign currency deposits & 640 & 747 & 680 & 574 & 1,288 & 1,783 & 1,330 & 1,673 & 1,664 & 1,580 & 1,696 & 1,693 & 1,760 & 1,808 \\
\hline M2 & 4,041 & 3,970 & 4,194 & 4,256 & 4,145 & 4,170 & 4,240 & 3,619 & 4,367 & 3,840 & 4,640 & 4,209 & 5,336 & 4,480 \\
\hline Currency in circulation & 450 & 442 & 447 & 444 & 478 & 478 & 469 & 413 & 475 & 424 & 471 & 441 & 507 & 455 \\
\hline Local currency deposits & 3,591 & 3,527 & 3,747 & 3,812 & 3,667 & 3,692 & 3,771 & 3,206 & 3,892 & 3,416 & 4,169 & 3,768 & 4,829 & 4,024 \\
\hline \multicolumn{15}{|l|}{ Central bank } \\
\hline Net foreign assets & 323 & 406 & 319 & 776 & 1,320 & 1,050 & 1,334 & 1,447 & 1,179 & 1,374 & 1,317 & 1,688 & 1,584 & 1,843 \\
\hline Foreign assets & 323 & 406 & 319 & 776 & 1,452 & 1,207 & 1,466 & 1,595 & 1,358 & 1,526 & 1,518 & 1,840 & 1,806 & 2,015 \\
\hline Of which: official reserves & 78 & 141 & 114 & 136 & 262 & 843 & 276 & 1,372 & 1,006 & 1,214 & 1,166 & 1,433 & 1,454 & 1,588 \\
\hline Foreign liabilities & 0 & 0 & 0 & 0 & 131 & 157 & 131 & 148 & 179 & 152 & 201 & 152 & 222 & 172 \\
\hline Net domestic assets & 966 & 735 & 963 & 495 & -169 & 70 & -188 & -396 & 87 & -108 & -7 & -376 & -233 & -492 \\
\hline Domestic credit & 1,103 & 895 & 1,094 & 1,092 & 549 & 851 & 532 & -56 & 703 & 133 & 629 & -115 & 423 & -219 \\
\hline Government (net) & 1,103 & 948 & 1,115 & 1,246 & 1,032 & 1,099 & 1,043 & 151 & 964 & 66 & 864 & 28 & 787 & 29 \\
\hline Commercial banks & 0 & 0 & 30 & -97 & $\ldots$ & -22 & $\ldots$ & 0 & $\ldots$ & 238 & $\ldots$ & 28 & $\ldots$ & -76 \\
\hline Other (parastatals) & 0 & -53 & -51 & -58 & -483 & -226 & -511 & -207 & -261 & -171 & -235 & -171 & -364 & -171 \\
\hline Other items, net & -137 & -160 & -131 & -597 & -718 & -780 & -720 & -340 & -616 & -241 & -636 & -261 & -656 & -273 \\
\hline Reserve money & 1,113 & 1,141 & 1,282 & 1,271 & 1,151 & 1,120 & 1,146 & 1,051 & 1,266 & 1,266 & 1,311 & 1,311 & 1,351 & 1,351 \\
\hline Currency in circulation & 450 & 442 & 447 & 444 & 478 & 478 & 469 & 413 & 475 & 424 & 471 & 441 & 507 & 455 \\
\hline Commercial bank reserves (includes cash in vault)' & 663 & 698 & 835 & 828 & 674 & 642 & 677 & 637 & 791 & 842 & 840 & 871 & 844 & 896 \\
\hline o.w. required reserves in foreign currency $y^{2}$ & & $\ldots$ & & & & $\ldots$ & $\ldots$ & $\ldots$ & $\ldots$ & 110 & & 204 & $\ldots$ & 223 \\
\hline \multicolumn{15}{|l|}{ Memorandum items: } \\
\hline Gross international reserves (US\$ millions) & 10 & 18 & 14 & 17 & 19 & 51 & 20 & 85 & 63 & 83 & 73 & 98 & 91 & 108 \\
\hline Foreign Currency Deposits (US\$ millions) & 80 & 94 & 85 & 70 & 92 & 108 & 95 & 104 & 104 & 108 & 106 & 116 & 110 & 123 \\
\hline M2 growth (12-month percent change) & -14.9 & -17.3 & -15.5 & 7.7 & 2.6 & 3.2 & 6.8 & -8.8 & 4.1 & -8.4 & 9.0 & -1.1 & 28.0 & 7.4 \\
\hline M3 growth (12-month percent change) & -7.6 & -9.5 & -7.9 & 8.0 & 16.1 & 27.2 & 18.1 & 12.2 & 23.7 & 11.2 & 31.2 & 22.2 & 19.2 & 5.6 \\
\hline Credit to the economy (12-month percent change) & 32.7 & 34.5 & 26.6 & 22.4 & 25.9 & 58.4 & 17.1 & 48.0 & 20.1 & 47.0 & 30.0 & 52.5 & 17.9 & 24.3 \\
\hline Reserve money (12-month percent change) & -23.1 & -32.4 & -21.0 & 17.4 & 3.4 & 0.6 & 0.5 & -7.9 & -1.3 & -1.3 & 3.1 & 3.2 & 20.6 & 20.6 \\
\hline Money multiplier (M2/reserve money) & 3.6 & 3.5 & 3.3 & 3.3 & 3.6 & 3.6 & 3.7 & 3.4 & 3.4 & 3.0 & 3.5 & 3.2 & 3.9 & 3.3 \\
\hline Money multiplier (M3/reserve money) & 4.2 & 4.1 & 3.8 & 3.8 & 1.9 & 5.3 & 2.0 & 5.0 & 4.8 & 4.3 & 4.8 & 4.5 & 5.3 & 4.7 \\
\hline Velocity (GDP/M2; end of period) & 1.5 & 2.2 & 2.2 & 2.2 & 0.0 & 2.2 & 2.0 & 2.8 & 2.0 & 2.3 & 2.0 & 2.3 & 1.8 & 2.3 \\
\hline Velocity (GDP/M3; end of period) & 1.5 & 1.5 & 1.5 & 1.5 & 1.5 & 1.5 & 1.5 & 1.9 & 1.4 & 1.6 & 1.4 & 1.6 & 1.4 & 1.6 \\
\hline Change in Net Credit to Government (change in quarter) & -213 & -56 & 157 & -39 & -137 & -162 & 103 & $-1,336$ & 101 & 184 & -316 & -360 & -244 & 4 \\
\hline
\end{tabular}

Sources: Central Bank of Seychelles, and IMF staff estimates and projections.

${ }^{1}$ Reserve requirements are being lowered from $13 \%$ to $12 \%$ in Q3.

${ }^{2}$ Starting April 2009, constitution in foreign currency of required reserves on foreign currency deposits were phased in. 
Table 4. Seychelles: Balance of Payments, 2007-10 (Millions of U.S. dollars)

\begin{tabular}{|c|c|c|c|c|c|c|c|c|c|c|c|}
\hline & \multirow[t]{2}{*}{2007} & \multicolumn{3}{|c|}{2008} & \multicolumn{4}{|c|}{2009} & \multicolumn{2}{|c|}{2009} & \multirow{2}{*}{$\begin{array}{l}2010 \\
\text { Proj. }\end{array}$} \\
\hline & & Prog. & $\begin{array}{c}\text { Country } \\
\text { Report No. } \\
09 / 121\end{array}$ & Prel. & Q1 (Prel.) & Q2 & Q3 & Q4 & Rev. Prog. & Proj. & \\
\hline $\begin{array}{l}\text { Current account } \\
\text { (Percent of GDP) }\end{array}$ & $\begin{array}{r}-213 \\
-21\end{array}$ & $\begin{array}{r}-247 \\
-29\end{array}$ & $\begin{array}{r}-267 \\
-32\end{array}$ & $\begin{array}{r}-420 \\
-46\end{array}$ & $\begin{array}{r}-23 \\
-3\end{array}$ & $\begin{array}{l}-65 \\
-10\end{array}$ & $\begin{array}{r}-41 \\
-6\end{array}$ & $\begin{array}{l}-72 \\
-11\end{array}$ & $\begin{array}{r}-177 \\
-29\end{array}$ & $\begin{array}{r}-202 \\
-30\end{array}$ & $\begin{array}{r}-252 \\
-33\end{array}$ \\
\hline $\begin{array}{l}\text { Trade balance } \\
\text { Exports of goods }\end{array}$ & $\begin{array}{r}-175 \\
392\end{array}$ & $\begin{array}{r}-216 \\
511\end{array}$ & $\begin{array}{r}-235 \\
491\end{array}$ & $\begin{array}{r}-386 \\
495\end{array}$ & $\begin{array}{r}-33 \\
89\end{array}$ & $\begin{array}{r}-50 \\
97\end{array}$ & $\begin{array}{r}-26 \\
89\end{array}$ & $\begin{array}{r}-38 \\
91\end{array}$ & $\begin{array}{r}-105 \\
370\end{array}$ & $\begin{array}{r}-146 \\
366\end{array}$ & $\begin{array}{r}-187 \\
400\end{array}$ \\
\hline Of which: oil re-exports & 190 & 293 & 260 & 271 & 32 & 54 & 59 & 41 & 132 & 186 & 233 \\
\hline Of which: tuna exports & 184 & 198 & 215 & 215 & 54 & 40 & 26 & 51 & 219 & 171 & 160 \\
\hline Oil imports & -236 & -337 & -339 & -391 & -51 & -29 & -72 & -56 & -143 & -207 & -250 \\
\hline FDI-related imports & -153 & -190 & -237 & -231 & -42 & -37 & -22 & -45 & -156 & -146 & -177 \\
\hline Other & -345 & -327 & -292 & -352 & -54 & -85 & -66 & -50 & -256 & -255 & -247 \\
\hline Exports of services & 481 & 505 & 523 & 512 & 98 & 70 & 110 & 94 & 383 & 372 & 409 \\
\hline Of which: tourism earnings & 284 & 303 & 276 & 267 & 64 & 28 & 66 & 50 & 207 & 209 & 223 \\
\hline Imports of services & -313 & -377 & -381 & -374 & -74 & -65 & -66 & -72 & -303 & -276 & -323 \\
\hline Income, net & -72 & -68 & -84 & -103 & -6 & -27 & -28 & -47 & -118 & -107 & -124 \\
\hline Of which: transfers of profits and dividends & -33 & -28 & -39 & -61 & -2 & -10 & -23 & -29 & -74 & -64 & -82 \\
\hline Of which: interest payments due ${ }^{1}$ & -33 & -33 & -33 & -33 & -4 & -15 & -3 & -14 & -32 & -36 & -33 \\
\hline Current transfers, net & 33 & 37 & 51 & 69 & 15 & 12 & 13 & 12 & 46 & 52 & 60 \\
\hline General government, net & 10 & 14 & 30 & 43 & 12 & 4 & 5 & 5 & 24 & 27 & 35 \\
\hline Other sectors, net & 23 & 23 & 22 & 26 & 3 & 8 & 8 & 7 & 22 & 25 & 25 \\
\hline Capital and financial account & 261 & 130 & 168 & 172 & 30 & 19 & 38 & 61 & 124 & 147 & 219 \\
\hline Capital account & 8 & 4 & 5 & 5 & 0 & 0 & 37 & 0 & 3 & 37 & 37 \\
\hline Financial account & 254 & 126 & 164 & 167 & 29 & 19 & 1 & 61 & 121 & 110 & 181 \\
\hline Direct investment, net & 225 & 296 & 354 & 346 & 53 & 46 & 27 & 56 & 200 & 183 & 213 \\
\hline Portfolio investment, net & 31 & 1 & 1 & 1 & 0 & 0 & 0 & 0 & 0 & 0 & 0 \\
\hline Other investment, net & -2 & -171 & -191 & -180 & -24 & -28 & -26 & 4 & -79 & -73 & -32 \\
\hline Assets & -66 & -13 & -78 & -5 & -31 & 6 & 2 & 13 & -16 & -10 & 6 \\
\hline Liabilities & 63 & -158 & -112 & -175 & 7 & -34 & -28 & -8 & -63 & -63 & -38 \\
\hline Government and government-guaranteed & 34 & -155 & -109 & -105 & -15 & -34 & -28 & -8 & -46 & -85 & -53 \\
\hline Disbursements & 101 & 26 & 73 & 84 & 0 & 0 & 24 & 5 & 39 & 29 & 39 \\
\hline Project loans & 101 & 24 & 73 & 84 & 0 & 0 & 5 & 5 & 20 & 10 & 20 \\
\hline Program loans & 0 & 2 & 0 & 0 & 0 & 0 & 19 & 0 & 19 & 19 & 19 \\
\hline Amortization (Principal payments) ${ }^{1,2}$ & & -181 & -181 & -189 & -15 & -35 & -15 & -15 & -85 & -81 & -56 \\
\hline Deferred interest ${ }^{1,2}$ & & & & & 0 & 1 & 0 & 2 & $\ldots$ & 3 & 1 \\
\hline Debt forgiveness & & & & & 0 & 0 & -37 & 0 & $\ldots$ & -37 & -37 \\
\hline Other & 29 & 5 & 4 & -70 & 22 & 0 & 0 & 0 & -17 & 22 & 15 \\
\hline Net errors and omissions & -8 & 14 & -17 & 101 & 23 & 0 & 0 & 0 & 0 & 23 & 0 \\
\hline Change in net international reserves (increase: - ) & 49 & 5 & -27 & -27 & -34 & 4 & -14 & -9 & -36 & -54 & -37 \\
\hline Change in gross official reserves (increase: - ) & 49 & -4 & -36 & -36 & -34 & 2 & -15 & -11 & -40 & -58 & -50 \\
\hline Prospective liabilities to IMF, net & 0 & 9 & 10 & 10 & 0 & 1 & 1 & 1 & 4 & 4 & 13 \\
\hline Other NFA (increase: -) & 0 & -54 & 0 & 0 & -10 & 0 & 0 & 0 & 0 & -10 & 0 \\
\hline Exceptional financing & -89 & 152 & 142 & 174 & 15 & 0 & 0 & 0 & & 15 & 0 \\
\hline Change in arrears (increase: +$)^{3,4}$ & -89 & 152 & 154 & 174 & 15 & -140 & 0 & 0 & 0 & -125 & 0 \\
\hline Clearance of arrears & & $\ldots$ & $\ldots$ & $\ldots$ & 0 & 140 & 0 & 0 & & 140 & 0 \\
\hline Financing gap & 0 & 0 & 0 & 0 & 0 & 43 & 17 & 21 & 89 & 80 & 70 \\
\hline \multicolumn{12}{|l|}{ Memorandum items: } \\
\hline Exports growth, percent & -8 & 30 & 25 & 26 & -24 & -20 & -37 & -21 & -25 & -26 & 9 \\
\hline Tourism growth, percent & 25 & 7 & -3 & -6 & -22 & -60 & 4 & 0 & -25 & -22 & 7 \\
\hline Imports growth, percent & 3 & 16 & 18 & 39 & -35 & -33 & -35 & -53 & -36 & -40 & 11 \\
\hline Exports, percent of GDP & 38 & 59 & 59 & 54 & $\ldots$ & $\ldots$ & $\ldots$ & $\ldots$ & 61 & 54 & 53 \\
\hline Imports, percent of GDP & 72 & 99 & 104 & 111 & $\ldots$ & $\ldots$ & $\ldots$ & $\ldots$ & 92 & 90 & 88 \\
\hline FDI, percent of GDP & 22 & 34 & 42 & 38 & $\cdots$ & $\cdots$ & $\cdots$ & $\cdots$ & 33 & 27 & 28 \\
\hline Gross international reserves (stock, e.o.p.) ${ }^{5}$ & 10 & 19 & 51 & 51 & 85 & 83 & 98 & 108 & 91 & 108 & 158 \\
\hline Months of prospective imports of goods and services & 0.1 & 0.2 & 0.7 & 0.7 & 1.0 & 1.0 & 1.2 & 1.3 & 1.2 & 1.3 & 1.8 \\
\hline Scheduled public external debt service ${ }^{1,6}$ & 100 & 222 & 222 & 221 & 19 & 49 & 18 & 28 & 118 & 114 & 88 \\
\hline (Percent of exports of goods and services) & 11.4 & 22 & 22 & 22 & 10 & 30 & 9 & 15 & 16 & 15 & 10.9 \\
\hline Public external debt ${ }^{1,4,6}$ & 710 & 840 & 763 & 802 & $\ldots$ & $\ldots$ & $\ldots$ & $\ldots$ & 806 & 733 & 695 \\
\hline (Percent of GDP) & 69.2 & 98 & 91 & 87 & $\ldots$ & $\ldots$ & $\ldots$ & $\ldots$ & 134 & 108 & 91.2 \\
\hline Of which: arrears ${ }^{1,3,4}$ & 160 & 331 & 310 & 333 & $\cdots$ & $\cdots$ & $\cdots$ & $\cdots$ & 310 & 195 & 195 \\
\hline (Percent of GDP) & 15.6 & 38 & 37 & 36 & $\cdots$ & $\ldots$ & $\cdots$ & $\ldots$ & 51 & 29 & 25.6 \\
\hline Cash available for debt service & $\ldots$ & $\ldots$ & $\ldots$ & $\ldots$ & 19 & 7 & 1 & 7 & 28 & 33 & 18 \\
\hline GDP & 1,026 & 859 & 834 & 921 & $\ldots$ & $\ldots$ & $\ldots$ & $\ldots$ & 603 & 679 & 762 \\
\hline
\end{tabular}

${ }^{1}$ Projections are based on identified new borrowing and rescheduled Paris Club debt. It assumes comparable cash flow treatment and identical reduction in the debt stock for South Africa, which also signed the agreement, and no debt restructuring for other countries. Arrears restructuring leads to higher rescheduled interest payments.

${ }^{2}$ Based on Paris Club agreement, the payments of principal and most interest during 2008 and through June 2009 are deferred.

${ }^{3}$ In 2008, includes accelerated promissory notes.

${ }^{4}$ Debt forgivenesss reflects: (i) the first stage of debt reduction (about 22.5) percent in July 2009; (ii) the second stage reduction (about 22.5 percent) in July 2010 on Paris Club debt. Assumes debt reduction comparable to the Paris Club agreement on South African debt.

${ }^{5}$ Starting with 2007, refers to CBS gross international reserves net of blocked deposits and project accounts.

${ }^{6}$ Preliminary and subject to reconciliation with creditors. Includes CBS external debt. 
Table 5. Seychelles: Financial Soundness Indicators for the Banking Sector, 2007 - $2009^{1}$

(Percent, eop)

\begin{tabular}{|c|c|c|c|c|c|c|c|c|c|}
\hline & \multicolumn{4}{|c|}{2007} & \multicolumn{4}{|c|}{2008} & \multirow{2}{*}{$\frac{2009}{\mathrm{Q} 1}$} \\
\hline & Q1 & Q2 & Q3 & Q4 & Q1 & Q2 & Q3 & Q4 & \\
\hline \multicolumn{10}{|l|}{${\text { Capital } \text { adequacy }^{2}}^{2}$} \\
\hline Regulatory capital to risk weighted assets & 20.4 & 21.0 & 18.2 & 18.2 & 18.4 & 17.6 & 17.9 & 19.4 & 21.8 \\
\hline Regulatory tier 1 capital to risk weighted assets & 14.8 & 16.1 & 14.8 & 12.9 & 12.9 & 14.0 & 13.4 & 10.4 & 11.1 \\
\hline Capital to assets (net worth) & 6.2 & 6.5 & 6.4 & 6.4 & 6.9 & 6.6 & 7.0 & 8.4 & 9.1 \\
\hline Net tangible capitalization ${ }^{3}$ & 6.2 & 6.6 & 6.5 & 6.5 & 7.0 & 6.6 & 7.1 & 8.4 & 9.2 \\
\hline \multicolumn{10}{|l|}{ Asset quality } \\
\hline Foreign exchange loans to total loans & 24.1 & 25.1 & 28.7 & 30.8 & 32.7 & 32.9 & 31.6 & 50.8 & 50.5 \\
\hline Non-performing loans to gross loans & 3.7 & 2.9 & 2.5 & 2.3 & 2.0 & 2.1 & 2.0 & 2.0 & 2.3 \\
\hline Provision as percentage of non-performing loans & 38.2 & 44.1 & 45.6 & 45.9 & 51.2 & 50.3 & 59.6 & 58.3 & 56.8 \\
\hline \multicolumn{10}{|l|}{ Earnings and profitability } \\
\hline Return on assets (annualised) & 3.9 & 3.4 & 5.0 & 6.6 & 4.3 & 3.4 & 2.8 & 16.1 & 5.5 \\
\hline Return on equity (annualised) & 59.1 & 50.4 & 68.3 & 93.0 & 59.5 & 47.9 & 39.3 & 199.4 & 57.6 \\
\hline Interest margin to gross income & 60.0 & 66.1 & 57.9 & 46.0 & 58.7 & 62.8 & 58.3 & 24.2 & 60.0 \\
\hline Non-interest expense to gross income & 35.5 & 39.7 & 33.3 & 32.3 & 33.2 & 43.5 & 49.4 & 17.8 & 35.4 \\
\hline Net interest margin (annualised) ${ }^{4}$ & 3.6 & 3.7 & 4.3 & 4.5 & 3.8 & 3.8 & 3.1 & 4.8 & 3.3 \\
\hline Net non interest margin (annualized) ${ }^{5}$ & 0.3 & -0.3 & 0.6 & 2.1 & 0.5 & -0.4 & -0.4 & 11.4 & 0.2 \\
\hline Expense to income & 48.5 & 53.5 & 44.8 & 42.2 & 46.6 & 55.9 & 62.5 & 25.5 & 50.5 \\
\hline Interest expense to gross income & 25.2 & 29.5 & 20.8 & 17.2 & 25.0 & 28.0 & 35.0 & 10.2 & 29.5 \\
\hline \multicolumn{10}{|l|}{ Liquidity } \\
\hline Core liquid assets to total assets ${ }^{6}$ & 19.2 & 18.6 & 11.4 & 10.0 & 30.8 & 34.6 & 36.4 & 37.8 & 36.0 \\
\hline Broad liquid assets to total assets ${ }^{7}$ & 54.8 & 55.8 & 44.3 & 39.4 & 59.2 & 60.9 & 60.0 & 55.7 & 50.8 \\
\hline Liquid assets (broad) to short term liabilities & 57.3 & 57.5 & 46.7 & 40.8 & 59.3 & 61.6 & 87.1 & 56.5 & 53.2 \\
\hline Liquid assets (broad) to total liabilities & 58.7 & 60.1 & 47.8 & 42.3 & 63.9 & 65.5 & 64.8 & 61.2 & 56.2 \\
\hline Liquid assets to deposit liabilities & 64.0 & 64.5 & 52.5 & 46.0 & 68.3 & 72.4 & 71.7 & 66.4 & 63.1 \\
\hline \multicolumn{10}{|l|}{ Foreign exchange exposure } \\
\hline Net open foreign exchange position to capital & 2.6 & 14.1 & 28.3 & 28.5 & 24.9 & 31.3 & 21.3 & 58.9 & 44.4 \\
\hline
\end{tabular}

Sourse: Central Bank of Seychelles

${ }^{1}$ Excluding purely offshore banks.

${ }^{2}$ Under new standards of provisioning and capital adequacy.

${ }^{3}$ Defined as: equity capital/(assets-interest in suspense-provisions)

${ }^{4}$ Defined as: (Interest income - interest expense)/average assets

${ }^{5}$ Defined as: (Non interest income - non interest expense)/average assets

${ }^{6}$ Core liquid assets include cash, balances with CBS and deposits with other banks

${ }^{7}$ Broad liquid assets include core liquid assets plus investments in government securities. 
Table 6. Seychelles: Balance of Payments, 2007-14 (Millions of U.S. dollars)

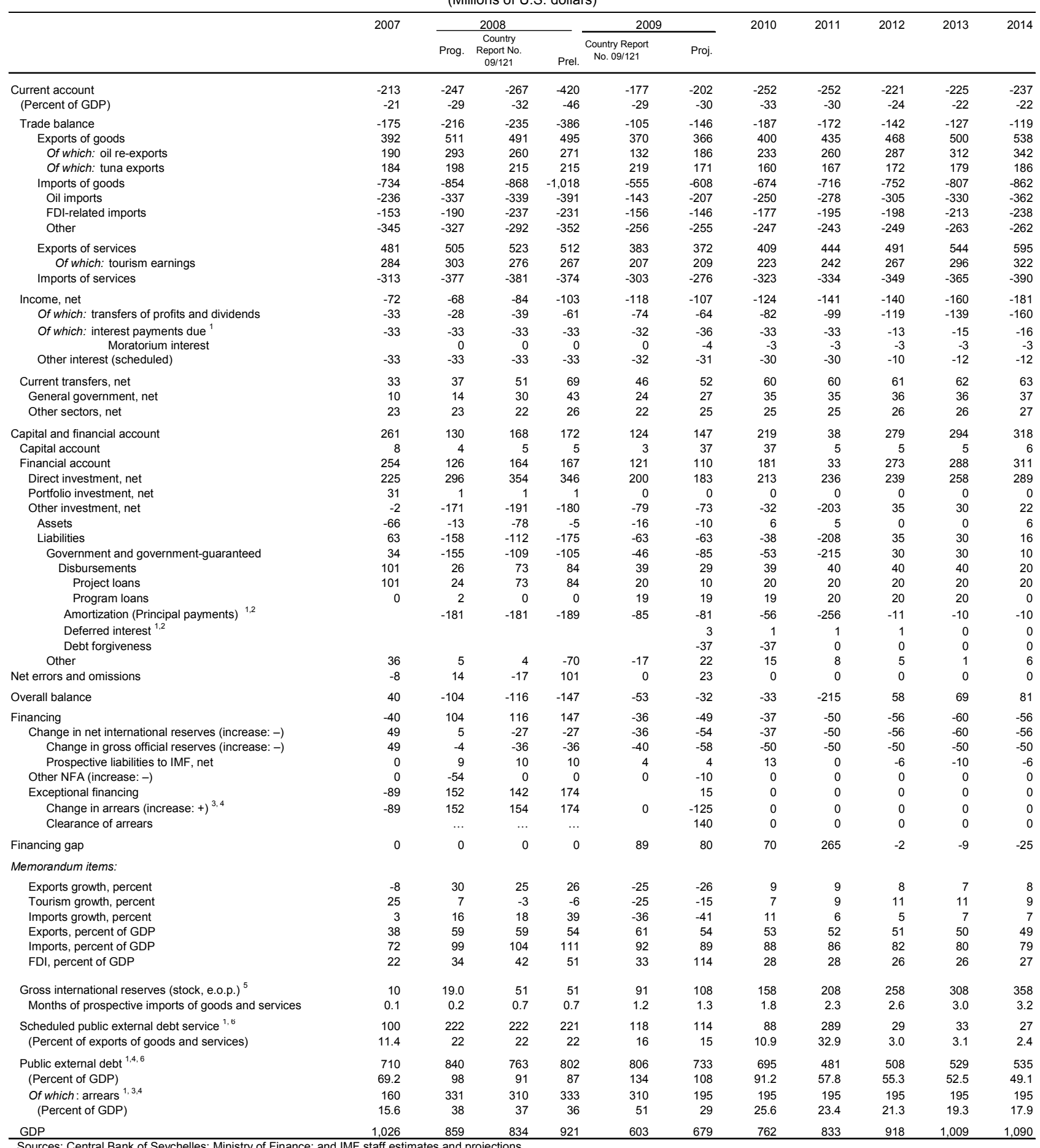

Sources: Central Bank of Seychelles; Ministry of Finance; and IMF staff estimates and projections.

${ }^{1}$ Projections are based on identified new borrowing and rescheduled Paris Club debt. It assumes comparable cash flow treatment and identical reduction in the debt stock for South Africa, which also signed the agreement, and no debt restructuring for other countries. Arrears restructuring leads to higher rescheduled interest payments.

${ }^{2}$ Based on Paris Club agreement, the payments of principal and most interest during 2008 and through June 2009 are deferred.

${ }^{3}$ In 2008, includes accelerated promissory notes.

${ }^{4}$ Debt forgivenesss reflects: (i) the first stage of debt reduction (about 22.5) percent in July 2009; (ii) the second stage reduction (about 22.5 percent) in July 2010 on Paris Club debt. Assumes debt reduction comparable to the Paris Club agreement on South African debt

${ }^{5}$ Starting with 2007, refers to CBS gross international reserves net of blocked deposits and project accounts.

${ }^{6}$ Preliminary and subject to reconciliation with creditors. Includes CBS external debt. 
Table 7. Seychelles: Projected Financing Requirements and Sources, 2008-19 (Millions of U.S. dollars)

\begin{tabular}{|c|c|c|c|c|c|c|c|c|c|}
\hline & 2008 & 2009 & 2010 & 2011 & 2012 & 2013 & 2014 & 2017 & 2019 \\
\hline \multicolumn{10}{|l|}{ External Financing } \\
\hline Gross financing requirements & 514.4 & 496.5 & 397.6 & 597.1 & 325.8 & 331.9 & 338.6 & 383.9 & 439.6 \\
\hline External current account deficit (excl. official transfers) & 463.0 & 228.3 & 286.5 & 287.7 & 256.7 & 260.9 & 273.6 & 327.0 & 381.3 \\
\hline Amortization of public debt (scheduled) ${ }^{1}$ & 188.7 & 85.1 & 60.8 & 259.1 & 13.2 & 10.4 & 8.9 & 6.9 & 8.3 \\
\hline Change in arrears (repayment $=+$ ) & -173.5 & 125.3 & 0.0 & 0.0 & 0.0 & 0.0 & 0.0 & 0.0 & 0.0 \\
\hline Gross reserves accumulation (increase $=+$ ) & 36.1 & 57.6 & 50.0 & 50.0 & 50.0 & 50.0 & 50.0 & 50.0 & 50.0 \\
\hline IMF repurchases and charges & 0.0 & 0.2 & 0.3 & 0.4 & 6.0 & 10.6 & 6.1 & 0.0 & 0.0 \\
\hline Available financing & 514.4 & 416.1 & 327.6 & 332.4 & 327.8 & 340.8 & 363.5 & 417.5 & 475.4 \\
\hline Foreign direct investment & 346.1 & 182.9 & 213.2 & 235.7 & 238.6 & 257.9 & 288.9 & 352.2 & 411.5 \\
\hline Disbursements of program support ${ }^{2}$ & 0.0 & 19.1 & 19.0 & 20.0 & 20.0 & 20.0 & 0.0 & 0.0 & 0.0 \\
\hline Multilateral & 0.0 & 19.1 & 19.0 & 20.0 & 20.0 & 20.0 & 0.0 & 0.0 & 0.0 \\
\hline Bilateral & 0.0 & 0.0 & 0.0 & 0.0 & 0.0 & 0.0 & 0.0 & 0.0 & 0.0 \\
\hline Disbursements of project loans & 84.2 & 10.1 & 20.0 & 20.0 & 20.0 & 20.0 & 20.0 & 20.6 & 20.6 \\
\hline Arrears clearance (inc. arrears forgiveness) & 0.0 & 140.0 & 0.0 & 0.0 & 0.0 & 0.0 & 0.0 & 0.0 & 0.0 \\
\hline IMF & 9.6 & 4.0 & 13.2 & 0.0 & 0.0 & 0.0 & 0.0 & 0.0 & 0.0 \\
\hline Other flows ${ }^{3}$ & 74.4 & 60.0 & 62.1 & 56.7 & 49.2 & 42.8 & 54.6 & 44.6 & 43.3 \\
\hline External flow financing gap & & 80.4 & 70.1 & 264.7 & -1.9 & -8.9 & -24.9 & -33.5 & -35.8 \\
\hline External arrears clearance ${ }^{3}$ & & 195.1 & & & & & & & \\
\hline Total financing need & & 275.5 & 70.1 & 264.7 & -1.9 & -8.9 & -24.9 & -33.5 & \\
\hline \multicolumn{10}{|l|}{ Fiscal Financing } \\
\hline \multicolumn{10}{|l|}{ Gross fiscal requirements } \\
\hline Debt service & 258.9 & 158.7 & 124.6 & 316.6 & 48.7 & 47.6 & 44.9 & 47.6 & 50.3 \\
\hline Interest payments due & 70.2 & 73.6 & 63.8 & 57.5 & 35.5 & 37.2 & 36.0 & 40.7 & 43.0 \\
\hline Domestic & 37.4 & 41.9 & 33.9 & 27.4 & 25.3 & 25.3 & 23.6 & 25.7 & 27.0 \\
\hline External & 32.8 & 31.7 & 29.9 & 30.1 & 10.2 & 12.0 & 12.4 & 15.0 & 16.0 \\
\hline External principal repayments due ${ }^{1}$ & 188.7 & 85.1 & 60.8 & 259.1 & 13.2 & 10.4 & 8.9 & 6.9 & 7.3 \\
\hline Source of funds & 258.9 & 86.6 & 54.5 & 71.6 & 62.4 & 64.3 & 70.1 & 85.3 & 92.3 \\
\hline Primary balance & 35.3 & 77.4 & 74.5 & 45.3 & 43.7 & 49.0 & 45.7 & 45.4 & 43.1 \\
\hline Privatization and long-term leases & 23.1 & 24.4 & 4.0 & 3.8 & 3.7 & 4.2 & 4.4 & 4.8 & 5.0 \\
\hline Domestic (net) & -7.3 & -38.0 & -62.9 & -17.5 & -24.9 & -29.0 & 0.0 & 14.5 & 25.4 \\
\hline External disbursements & 84.2 & 29.1 & 39.0 & 40.0 & 40.0 & 40.0 & 20.0 & 20.6 & 20.6 \\
\hline Change in fiscal arrears (net) & 137.1 & 5.5 & 0.0 & 0.0 & 0.0 & 0.0 & 0.0 & 0.0 & 0.0 \\
\hline Other ${ }^{4}$ & -13.4 & -11.9 & 0.0 & 0.0 & 0.0 & 0.0 & 0.0 & 0.0 & 0.0 \\
\hline Fiscal flow financing gap & & 72.2 & 70.1 & 244.9 & -13.8 & -16.7 & -25.1 & -37.7 & -42.1 \\
\hline External arrears clearance & & 195.1 & & & & & & & \\
\hline Total financing need & & 267.3 & 70.1 & 244.9 & -13.8 & -16.7 & -25.1 & -37.7 & -42.1 \\
\hline
\end{tabular}

Sources: Central Bank of Seychelles; Ministry of Finance; and IMF staff estimates and projections.

${ }^{1}$ On existing debt and disbursements of new assumed debt, excluding IMF assuming no debt restructuring and no repayment of arrears.

${ }^{2}$ Includes loans and grants.

${ }^{3}$ Includes all other net financial flows and errors and omissions.

${ }^{4}$ Statistical discrepancy. 
Table 8. Seychelles: Fund Disbursements and Timing of Reviews Under the Two-Year Stand-By Arrangement, 2008-10

\begin{tabular}{|c|c|c|c|c|}
\hline \multirow{2}{*}{ Review } & \multirow{2}{*}{ Date availability } & \multirow[b]{2}{*}{$\begin{array}{l}\text { Board approval of the SBA arrangement - } \\
\text { disbursed }\end{array}$} & \multicolumn{2}{|c|}{$\frac{\text { Amount }}{\text { (millions of SDR) (percent of quota) }}$} \\
\hline & & & 6.16 & 70 \\
\hline First & Disbursed & $\begin{array}{l}\text { Completion of first review (end-December } 2008 \\
\text { quantitative performance criteria) }\end{array}$ & 0.88 & 10 \\
\hline \multirow[t]{2}{*}{ Second } & June 15, 2009 & $\begin{array}{l}\text { Completion of second review (end-March } 2009 \\
\text { quantitative performance criteria) }\end{array}$ & 0.88 & 10 \\
\hline & August 15, 2009 & $\begin{array}{l}\text { Compliance with end-June } 2009 \text { quantitative and } \\
\text { structural performance criteria }\end{array}$ & 0.88 & 10 \\
\hline \multirow[t]{2}{*}{ Third } & December 15, 2009 & $\begin{array}{l}\text { Completion of third review (end-September } 2009 \\
\text { quantitative performance criteria) }\end{array}$ & 2.20 & 25 \\
\hline & March 15, 2010 & $\begin{array}{l}\text { Compliance with end-December } 2009 \text { quantitative } \\
\text { performance criteria }\end{array}$ & 2.20 & 25 \\
\hline \multirow[t]{2}{*}{ Fourth } & June 15, 2010 & $\begin{array}{l}\text { Completion of fourth review (end-March } 2010 \\
\text { quantitative performance criteria) }\end{array}$ & 2.20 & 25 \\
\hline & September 15, 2010 & $\begin{array}{l}\text { Compliance with end-June } 2010 \text { quantitative } \\
\text { performance criteria }\end{array}$ & 2.20 & 25 \\
\hline
\end{tabular}

Source: IMF staff. 
Table 9. Seychelles: Indicators of Fund Credit, 2008-15

(Millions of SDR)

\begin{tabular}{|c|c|c|c|c|c|c|c|c|}
\hline & 2008 & 2009 & 2010 & 2011 & 2012 & 2013 & 2014 & 2015 \\
\hline & \multicolumn{8}{|c|}{ Projections } \\
\hline \multicolumn{9}{|l|}{ Existing Fund credit } \\
\hline Stock ${ }^{1}$ & 6.2 & 6.2 & 6.2 & 6.2 & 3.1 & 0.0 & 0.0 & 0.0 \\
\hline Obligation & 0.0 & 0.1 & 0.1 & 0.1 & 3.2 & 3.1 & 0.0 & 0.0 \\
\hline \multicolumn{9}{|l|}{ Proposed SBA } \\
\hline Disbursements & 6.2 & 2.6 & 8.8 & 0.0 & 0.0 & 0.0 & 0.0 & 0.0 \\
\hline Obligations $^{2}$ & 0.0 & 0.0 & 0.1 & 0.2 & 0.8 & 3.9 & 4.0 & 0.8 \\
\hline Principal (repayments/repurchases) & 0.0 & 0.0 & 0.0 & 0.0 & 0.7 & 3.8 & 4.0 & 0.8 \\
\hline Charges and interest & 0.0 & 0.1 & 0.2 & 0.3 & 0.2 & 0.2 & 0.1 & 0.0 \\
\hline Stock of existing and prospective Fund credit ${ }^{1}$ & 6.2 & 11.0 & 15.4 & 15.4 & 11.7 & 4.8 & 0.8 & 0.0 \\
\hline Percent of quota & 70.0 & 125.0 & 175.0 & 175.0 & 132.5 & 54.4 & 9.4 & 0.0 \\
\hline Percent of GDP & 1.0 & 2.4 & 3.0 & 2.8 & 1.9 & 0.7 & 0.1 & 0.0 \\
\hline Percent of exports of goods and services & 0.9 & 2.2 & 2.9 & 2.6 & 1.8 & 0.7 & 0.1 & 0.0 \\
\hline Percent of gross reserves & 18.4 & 15.3 & 14.6 & 11.1 & 6.8 & 2.3 & 0.3 & 0.0 \\
\hline \multicolumn{9}{|l|}{ Obligations to the Fund from existing and prospective } \\
\hline Fund arrangements & 0.0 & 0.0 & 0.1 & 0.2 & 0.8 & 3.9 & 4.0 & 0.8 \\
\hline Percent of quota & 0.3 & 0.5 & 1.5 & 1.7 & 9.2 & 44.7 & 45.7 & 9.5 \\
\hline Percent of GDP & 0.0 & 0.0 & 0.0 & 0.0 & 0.1 & 0.6 & 0.6 & 0.1 \\
\hline Percent of exports of goods and services & 0.0 & 0.0 & 0.0 & 0.0 & 0.1 & 0.6 & 0.5 & 0.1 \\
\hline Percent of gross reserves & 0.1 & 0.1 & 0.1 & 0.1 & 0.5 & 1.9 & 1.7 & 0.3 \\
\hline
\end{tabular}

Sources: IMF Finance Department; and IMF staff estimates and projections.

${ }^{1}$ End of period.

${ }^{2}$ Repayment schedule based on repurchase obligations. 
Figure 2. Seychelles: Macroeconomic Developments
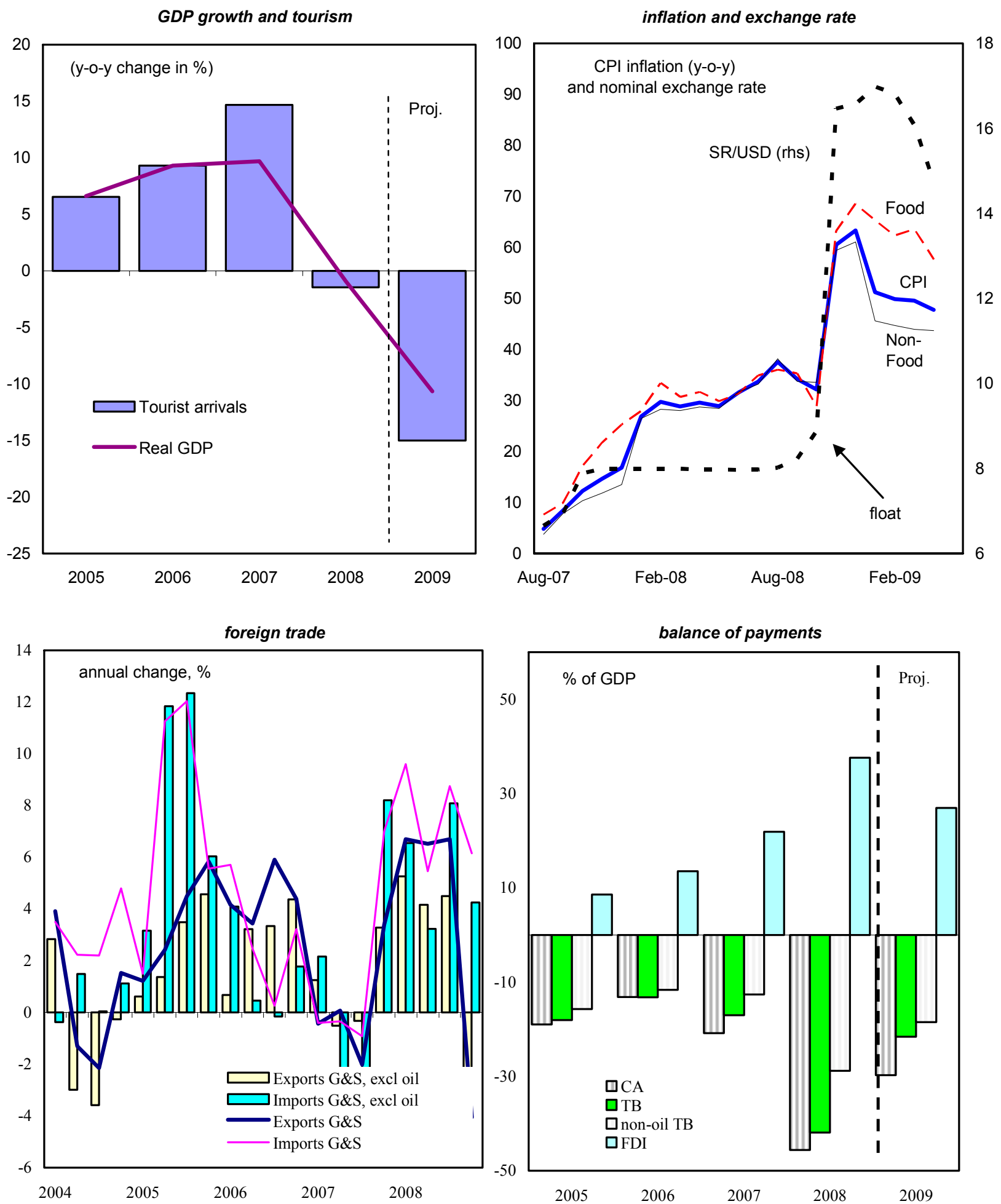

Source: Seychelles authorities; and Fund staff estimates. 
Figure 3. Seychelles: Fiscal Sector
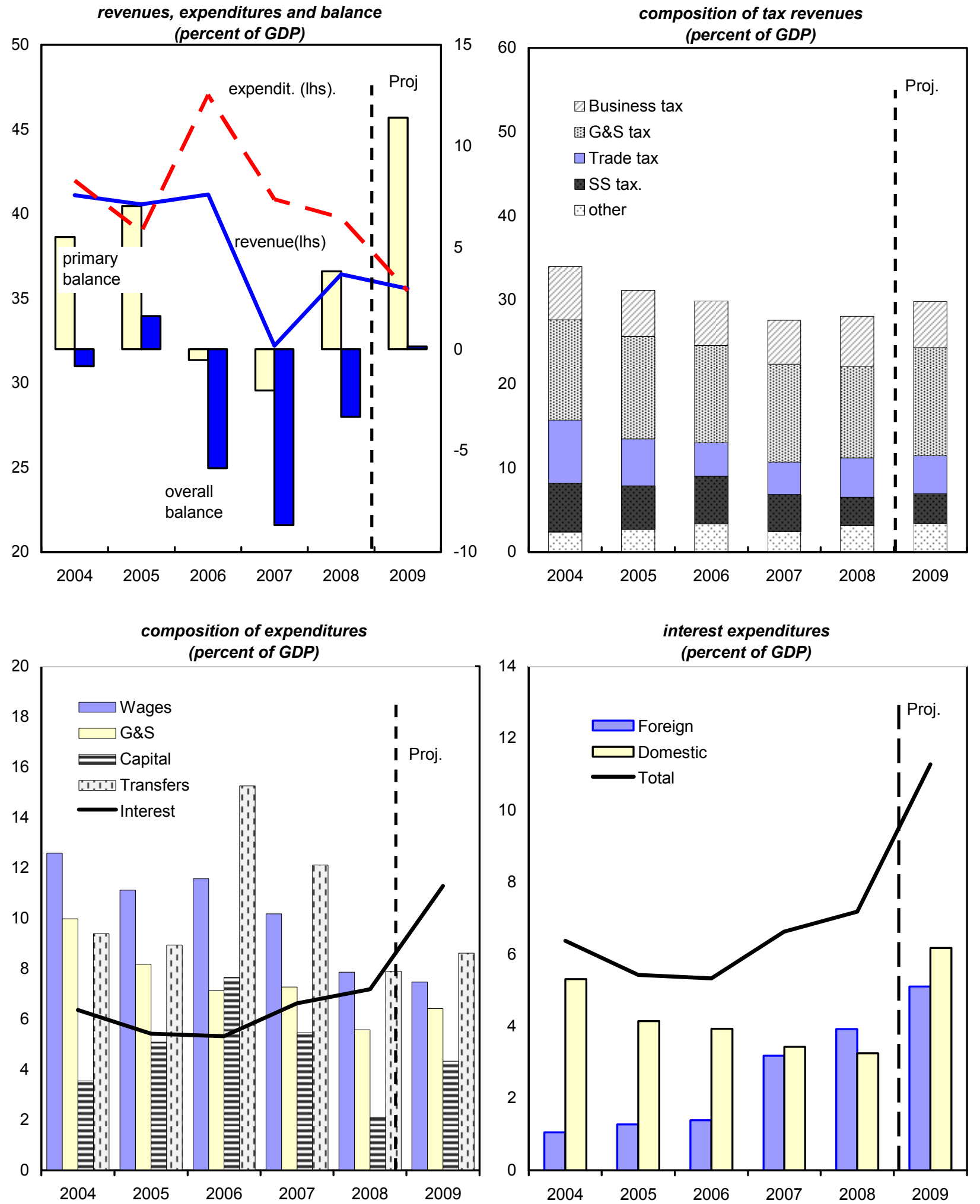

Source: Seychelles authorities; and Fund staff estimates. 
Figure 4. Seychelles: Monetary Sector
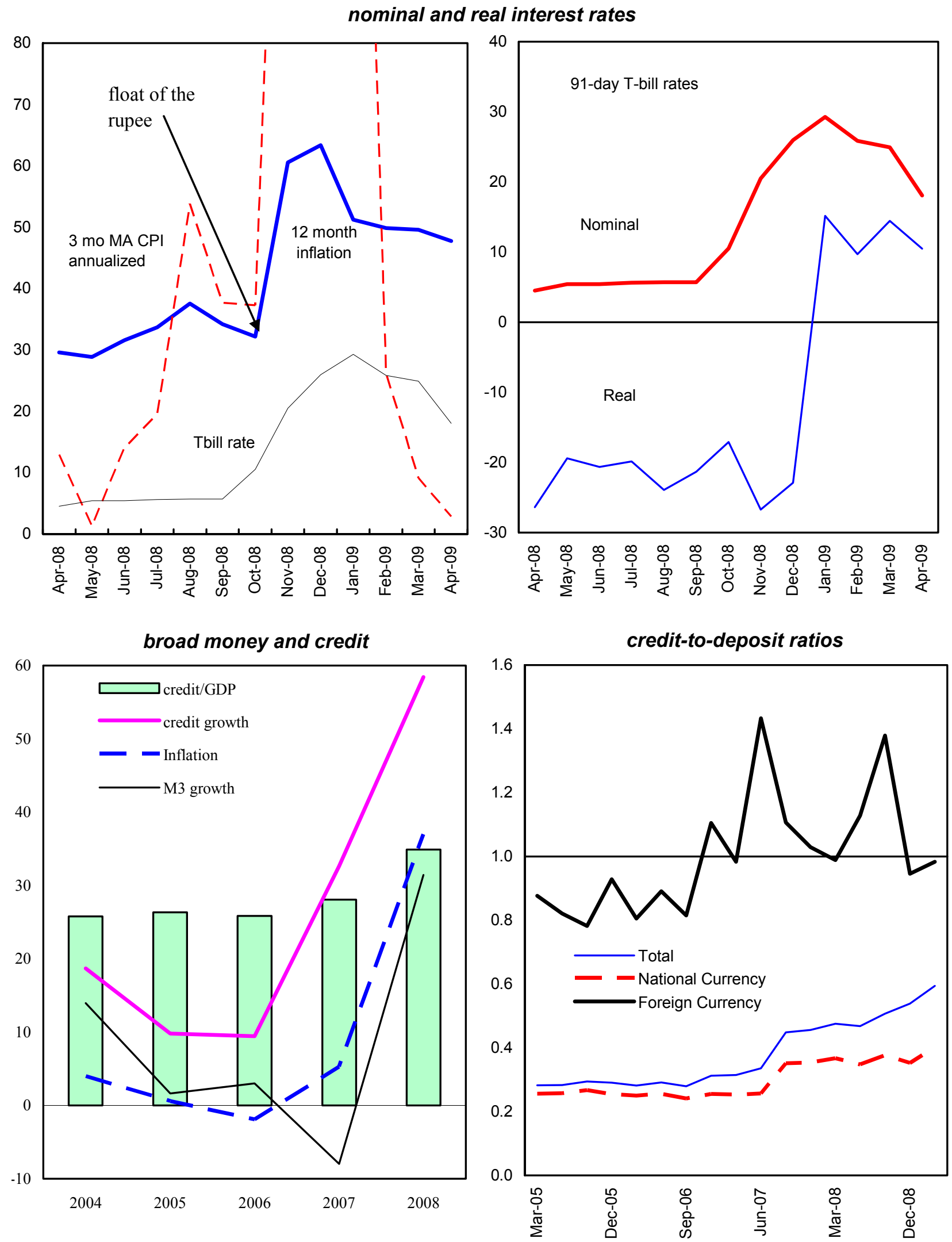

Source: Seychelles authorities; and Fund staff estimates 


\section{APPENDIX I \\ SEYCHELLES: LETTER OF INTENT}

June 16, 2009

Mr. Dominique Strauss-Kahn

Managing Director

International Monetary Fund

Dear Mr. Strauss-Kahn:

1. The attached Updated Memorandum of Economic and Financial Policies for 2009 (MEFP) describes Seychelles' performance in the first quarter of 2009 and policies for the remainder of the year. We request completion of the second review under the Stand-by arrangement and the release of the third tranche of SDR 0.88 million (10 percent of quota).

2. All the quantitative performance criteria (PCs) at end-March 2009 were met. One of the two structural benchmarks at end-April was observed. The second was missed due to a delay in the approval of new bank capitalization norms, which was implemented in May. We are making progress towards meeting the structural performance criterion and benchmarks for endJune and end-September. We are also moving ahead with our external debt restructuring strategy and are committed to achieving comparable treatment to the Paris Club agreement with all creditors.

3. We believe that the economic and financial policies set forth in the MEFP are sufficient to ensure that the objectives of the program will be met. We stand ready to take any further measures that may prove necessary to meet our objectives. We will consult with the Fund on the adoption of these measures, and in advance of revisions to the policies contained in the MEFP, in accordance with the Fund's policies on such consultations. We remain committed to full implementation of the recommendations of the Fund's safeguards assessment of the Central Bank of Seychelles (CBS).

4. We have begun to elaborate a comprehensive medium-term structural reform strategy, in collaboration with our international partners, in support of which we intend to request a 3-year arrangement under the Extended Fund Facility in late 2009 coinciding with our 2010 budget.

5. In line with our commitment to transparency, we request the IMF publish this letter, the MEFP, the technical memorandum of understanding (TMU), and the staff report. We will simultaneously publish these documents in Seychelles.

Sincerely yours,

$/ \mathrm{s} /$

James Alix Michel

President

Republic of Seychelles

Attachments: MEFP and TMU 


\section{ATtACHMENT I}

\section{Seychelles: Memorandum of Economic And Financial Policies for 2009 (UPDATED FOR THE SECOND PROGRAM REVIEW)}

\section{INTRODUCTION}

1. This document updates the MEFP of March 13, 2009 for the first program review. It reviews macroeconomic and financial performance in the first quarter of 2009 and describes our policies and reform measures for the remainder of the year.

\section{Recent Developments and Performance Under the Program}

2. The economy has stabilized more rapidly than anticipated so far in $\mathbf{2 0 0 9}$. Supported by tight monetary and fiscal policies, inflation has declined sharply to 2.4 percent in the first four months of 2009 (annualized), aided by sharp declines in food and petroleum prices. The rupee, which stabilized following the float at around SR16-17/US\$, has appreciated significantly in the second quarter. Nominal interest rates have been declining steadily from their highs as inflation has fallen and real interest rates had risen to positive levels early in the year.

3. The global economic recession is nonetheless pressing on Seychelles, with tourism earnings in particular falling significantly in the first quarter. Imports fell faster than exports, partly due to the decline in FDI and in private incomes. At the same time, while tourism earnings weakened, exports of tuna have held up well. Gross official reserves rose to US\$85 million ( 1 month of imports, well above the program target) at end-March on some one-off inflows and the unblocking of foreign asset accounts.

4. Fiscal policy over performed the program target in the first quarter on a more rapid repayment by SEYPEC of its financing facility, tightened expenditure controls (notably through the strengthened procedures under the new Procurement Act), the non use of the public financial institutions recapitalization contingency, and higher fishing license fees. Revenues also held up well, exceeding program projections. The primary fiscal balance was SR784 million (8.1 percent of GDP). A qualified financial controller was appointed to the Ministry of Health to strengthen expenditure controls, an auditor was hired to conduct management audits of the largest public enterprises and a public enterprise monitoring unit was established at the Ministry of Finance to support public enterprise reform. The first steps to implement a treasury single account were taken and an upgraded public debt report (for 2008) was produced.

5. Monetary policy was kept tight and the reserve money target was observed, supported by fiscal over performance. Positive real interest rates have been instrumental in containing inflationary pressures and limiting the second round effects of the depreciation of the rupee on prices. As an early sign of financial stabilization, interest rates on government 
paper declined notably from the peak 30 percent in January 2009 to about 15 percent in May 2009. The CBS has implemented with success the new reserve money management framework, relying on indirect policy instruments, such as the deposit auction facility with commercial banks. To allow for more operational flexibility in commercial banks liquidity management, the required reserves maintenance period has been increased to two weeks, while gradually shifting that on FX deposits to foreign exchange. The 91-day Treasury bill rate has become a benchmark rate.

\section{All quantitative performance criteria for end-March were comfortably met} (Table 1) and one of the structural benchmarks for end-April was observed while part of the second one was missed as bank capitalization norms were approved with a delay (Table 2). Table 3 lists the measures that were implemented (additional to the structural benchmarks) in the first four months of 2009.

\section{The passage of the new central bank act was an important step towards} modernizing monetary policy. In line with best international practices, price stability is now the primary objective of monetary policy. The law also strengthens the independence and accountability of the CBS, introduces new rules for CBS profit calculation and distribution, and puts in place a new capital structure that adjusts dynamically with changing monetary liabilities of the central bank.

\section{On April 16, 2009 Paris Club creditors (with the participation of South Africa) granted an exceptional debt treatment to Seychelles under the Evian approach. US\$140} million of arrears were regularized and the consolidated debt stock will be reduced by 45 percent in nominal terms in two tranches: the first, after the completion of the second program review (July 2009) and the second after the completion of the first review under a successor arrangement with the IMF (expected around June 2010) and satisfactory implementation of the comparability of treatment clause with respect to the agreements reached with other creditors. The remaining amounts will be rescheduled over 18 years, including 5 years of grace. A "goodwill" payment of US\$1 million will be made by endJune.

9. We are continuing our good faith efforts with other creditors to agree a restructuring on comparable terms. We have completed a second road show for commercial creditors and are maintaining a dialogue with creditors to inform the outlines of a restructuring proposal. We have been disseminating data and information relevant to our creditors through a dedicated website, including up to date estimates of our external financing gap and extent of the necessary debt service reduction. However, despite our best efforts, we were unable to stop payments by third parties to two of our external creditors through escrow accounts outside of Government control. We have instructed the third parties to cease making payments into these accounts, consistent with our commitment to intercreditor equity. 


\section{Updated Macroeconomic ANd Financial Program for 2009}

10. Building on the strong performance in the first quarter, our macroeconomic policies for the remainder of $\mathbf{2 0 0 9}$ aim to consolidate the gains in macrostabilization, notably on low inflation. We intend to lock-in most of the fiscal overperformance of the first quarter, while allowing for a measured easing of monetary policy. However, driven by a dropoff in tourism and construction, real GDP is projected to contract by almost 11 percent in 2009, with a modest recovery starting from 2010 . On the back of weakening domestic demand and declining FDI, the current account deficit is expected to improve to about 27 percent of GDP in 2009 .

11. Our reform objectives are to consolidate macroeconomic stability, and lay the foundation for economic recovery and sustained growth. We will continue to remove distortions, modernize economic laws and regulations, and reduce the role of the state in the economy. We will implement a significant reinforcement of monitoring and control over parastatal bodies to address major financial risks to the stability of public finances. We will also implement a fundamental reform of tax policy and administration. We will work to strengthen and modernize the financial system. We will also continue our good faith efforts to restructure our external public debt, building on the agreement with Paris Club creditors.

\section{A. Fiscal Policy}

12. To bank the gains in macro stabilization, the program aims to lock-in most of the over-performance achieved in the first quarter. The primary fiscal surplus will reach 11.4 percent of GDP. We are taking measures to further strengthen revenue collection, reinforce control of public expenditure, and raise the efficiency and transparency of public finance. At the same time, the contingency for the recapitalization of the public financial institutions has been reduced by two-thirds ( 2 percent of GDP), reflecting reduced potential need. These savings have been reallocated to meet unforeseen expenditure demands, including to respond to the threat of piracy in Seychelles' territorial waters, to cover financial losses of the public utilities corporation while it is being reformed, and to meet pressing social needs. Overall, the primary surplus, together with declining interest rates, will allow a reduction of domestic financing by the equivalent of 5.6 percent of GDP.

\section{Tax reform}

13. We are preparing to launch a fundamental reform of the tax system with technical support from the IMF. Our objective is to have a simple, fair, and equitable system, which will promote growth, improve self-compliance, and level the playing field for investors. Our tax reform strategy will have three major components:

- $\quad$ Broadening of the business tax base by removing exemptions and sectoral preferences (tourism and fishing); lowering/eliminating thresholds and accelerated capital depreciation schedules. Commensurately, rates will be harmonized at lower levels. This reform will be launched with the 2010 budget. 
- Introduction of a personal income tax to replace the social security contributions and other taxes on personal earnings to improve transparency, efficiency, and fairness. We expect to introduce the personal income tax by July 2010.

- Introduction of a single rate VAT by January 2012, replacing the current multiple rate GST, to improve the efficiency of the indirect tax system and external competitiveness.

The tax incentives under the Tourism Incentive Act, Agriculture and Fisheries Incentive Act, and the Investment Promotion Act will be eliminated by January 1, 2010 with the introduction of the new tax code. New concessions under these acts will have a duration limited to July 2010 at most. We will seek to transition companies with existing concessions agreements into the new tax system. We will also introduce measures to bolster tax administration and have requested technical assistance from the IMF in this regard.

\begin{tabular}{|c|c|}
\hline \multirow{2}{*}{$\begin{array}{l}\text { Action } \\
\text { Administration }\end{array}$} & Timing \\
\hline & \\
\hline $\begin{array}{l}\text { - Introduce a "Valid Tax Invoice" system to provide for an audit trail } \\
\text { for the claiming of deductions for business tax as well as input tax } \\
\text { credits for the new VAT. }\end{array}$ & July 2009 \\
\hline $\begin{array}{l}\text { - Grant Seychelles Revenue Commission (SRC) autonomy from public } \\
\text { administration (DPA) human resource norms }\end{array}$ & June 2009 \\
\hline $\begin{array}{l}\text { - Introduce a Tax Administration Act governing all administrative } \\
\text { issues surrounding legislation administered by the SRC. }\end{array}$ & January 2010 \\
\hline - $\quad$ Eliminate retail shops from the International Trade Zone (ITZ) & July 2010 \\
\hline $\begin{array}{l}\text { - Introduction of a Seychelles business number to serve as an account } \\
\text { number for all revenue streams within Seychelles. }\end{array}$ & January 2010 \\
\hline $\begin{array}{l}\text { - Introduce legislation to facilitate a single consolidated client account } \\
\text { and introduce provisions within such legislation where liabilities and } \\
\text { refunds may be netted off. }\end{array}$ & July 2010 \\
\hline $\begin{array}{l}\text { - Integrate the Seychelles Licensing Authority within the SRC in order } \\
\text { to achieve synergies for both government and clients }\end{array}$ & January 2010 \\
\hline $\begin{array}{l}\text { - Outsource the Seychelles Pension Fund collection and compliance } \\
\text { functions to the SRC to achieve synergies for both Government and } \\
\text { clients }\end{array}$ & January 2010 \\
\hline $\begin{array}{l}\text { - Outsource the SRC "point of contact" (cashier) functionalities to a } \\
\text { suitable third party with a large geographic footprint in order to } \\
\text { further enhance the "one-stop-shop" principle for the public }\end{array}$ & July 2010 \\
\hline $\begin{array}{l}\text { - Develop a Client Management System (CMS) for the SRC so it is in a } \\
\text { position to manage the revenue collection effectively and with } \\
\text { integrity }\end{array}$ & $\begin{array}{l}\text { July } 2009 \text { to } \\
\text { January } 2012\end{array}$ \\
\hline $\begin{array}{l}\text { - Introducing new legislation to strengthen the current Tax Agent Board } \\
\text { by providing it with more effective management capabilities as well } \\
\text { as limited arbitration functions }\end{array}$ & January 2010 \\
\hline $\begin{array}{l}\text { - Introduce legislation to mandate that all revenue collected by the SRC } \\
\text { be posted to the Government's consolidated revenue account. }\end{array}$ & September 2009 \\
\hline
\end{tabular}




\begin{tabular}{|c|c|}
\hline Business Tax & \\
\hline - Amend the Business Tax Act in line with the tax reform strategy & December 2009 \\
\hline Reduce the tax free threshold to international standards & January 2010 \\
\hline $\begin{array}{l}\text { - Introduce legislation to facilitate a Uniform Capital Allowance } \\
\text { system, annul all accelerated depreciation rules and cap all } \\
\text { depreciation allowances at } 100 \text { percent of cost }\end{array}$ & January 2010 \\
\hline $\begin{array}{l}\text { - Introduce simple Capital Gains legislation to capture large capital } \\
\text { profits currently un-taxed. }\end{array}$ & July 2010 \\
\hline $\begin{array}{l}\text { - Replace the current withholding and DAS provisions in the Business } \\
\text { Tax Act with a pay as you go instalment (PAYGI) system. }\end{array}$ & January 2010 \\
\hline $\begin{array}{l}\text { - Introduce a thin capitalisation ratio of } 3 \text { to } 1 \text { within the Business Tax } \\
\text { act for defined types of businesses }\end{array}$ & January 2010 \\
\hline $\begin{array}{l}\text { Bring the tax rate for tourism, fisheries, agriculture and other } \\
\text { concessionary industries in line with the normal tax rate. }\end{array}$ & January 2010 \\
\hline Personal Income tax (Pay as you go withholding) & \\
\hline $\begin{array}{l}\text { - Introduce new legislation to replace SSF contribution with Pay As } \\
\text { You Go withholding (PAYGW) system }\end{array}$ & July 2010 \\
\hline $\begin{array}{l}\text { - Introduce simple Fringe Benefit legislation based on the current } \\
\text { interpretation of non-monetary benefits within the Social Security Act. }\end{array}$ & July 2010 \\
\hline Indirect taxation & \\
\hline $\begin{array}{l}\text { - Introduce GST on licenses provided by the Seychelles Licensing } \\
\text { Authority }\end{array}$ & January 2010 \\
\hline - $\quad$ Introduce GST on all consultancy services & January 2010 \\
\hline - Eliminate the retail mark-up to calculate the GST & January 2010 \\
\hline $\begin{array}{l}\text { - Change current GST legislation to include all goods and services } \\
\text { unless exempt (create positive list) }\end{array}$ & January 2010 \\
\hline $\begin{array}{l}\text { - Replace current GST legislation and replace it with a full GST (VAT) } \\
\text { system including the availability of input tax credits }\end{array}$ & January 2012 \\
\hline Trade Taxes & \\
\hline $\begin{array}{l}\text { - Introduce the new Customs Management Act including HS codes: } \\
\text { "Seychelles Taxation System (Customs Management) Act 2010" }\end{array}$ & January 2010 \\
\hline Rationalize all trade tax rates in line with international commitments & January 2010 \\
\hline
\end{tabular}

14. We will implement expenditure rationalization measures recommended in the World Bank's Public Expenditure Review (PER), initially focusing on the health and education sectors. Based on the findings of the high-level government task force, the functions of the civil service will be streamlined with a view to eliminating duplication and outsourcing ancillary services to the private sector.

\section{We will continue to strengthen the public financial management framework.}

Modernizing and strengthening the treasury function will be important to consolidate and optimize the use of government cash balances, and improve oversight by the Ministry of Finance of spending units. For that purpose, we have begun moving to a Treasury Single Account and have already closed most of the ministerial and parastatal entity accounts in commercial banks. We will ensure that the recently created financial analysis branch within the SRC has the staffing and tools to adequately assess all investment proposals. 
16. Rationalizing the size of public sector and greatly increasing the financial control and monitoring of parastatal finances is a key building block of our reform program. In recognition of the need to improve monitoring and control of the public enterprises, a detailed work plan for the new Public Enterprise Monitoring Division has been approved. The key emphasis of the Division will be to drive improved operational efficiency; significantly strengthened governance; reduction of dependence on public funding and financial risks to the state. The Division will monitor all government owned organizations and will undertake a strategic assessment that will make recommendations on ownership and corporate structure to improve efficiency in the provision of essential services and ensure Government receives maximum returns on its investments and value for money. The Division will achieve these objectives through a combination of improvements in the legal framework, management reviews, external audits, increased and improved reporting obligations, development and introduction of policies and guidelines and strategic assessments of the government's interest in parastatals.

\begin{tabular}{|c|c|}
\hline Action & Timing \\
\hline \multicolumn{2}{|l|}{ Budget } \\
\hline - $\quad$ Adopt a treasury single account & September 2009 \\
\hline \multicolumn{2}{|l|}{ Pubic enterprises and entities } \\
\hline $\begin{array}{l}\text { - Collect monthly reports from public enterprises and analyze on a } \\
\text { quarterly basis }\end{array}$ & July 2009 \\
\hline $\begin{array}{l}\text { Launch a tender for an advisor for the privatization of Nouvobanq } \\
\text { and Seychelles Savings Bank }\end{array}$ & September 2009 \\
\hline - $\quad$ Develop corporate governance guidelines for public entities & August 2009 \\
\hline $\begin{array}{l}\text { - Complete external audits of the } 2008 \text { accounts of seven major } \\
\text { public enterprises (PUC, STC, SEYPEC, SCAA, IDC, Air } \\
\text { Seychelles and Nouvobanq) in accordance with international } \\
\text { audit standards }\end{array}$ & September 2009 \\
\hline - $\quad$ Introduce a new Public Enterprise Monitoring and Control Act & September 2009 \\
\hline - $\quad$ Prepare a strategic assessment for each major public enterprise & September 2009 \\
\hline $\begin{array}{l}\text { Ministry of finance to conduct management audits of major } \\
\text { public enterprises }\end{array}$ & $2009-10$ \\
\hline $\begin{array}{l}\text { Develop reform action plans for major public enterprises } \\
\text { following the external and management audits. }\end{array}$ & $2009-10$ \\
\hline $\begin{array}{l}\text { - Introduce policies to guide management of public entity debt in } \\
\text { line with the debt strategy }\end{array}$ & December 2009 \\
\hline \multicolumn{2}{|l|}{ Public debt } \\
\hline - $\quad$ Prepare a medium-term debt strategy & November 2009 \\
\hline $\begin{array}{l}\text { - Prepare an annual borrowing plan to be submitted with the } 2010 \\
\text { budget }\end{array}$ & November 2009 \\
\hline - $\quad$ Begin establishing performance benchmarks for public enterprises & December 2009 \\
\hline
\end{tabular}




\section{B. Public Debt Restructuring Strategy}

17. We are committed to continuing our good faith efforts to achieve a comprehensive public external debt restructuring, consistent with our medium-term payments capacity and the Fund's lending into arrears policy. We will ensure information transparency, inter-creditor equity, and dialogue with all creditor groups. As was recognized by Paris Club creditors, immediate and sizable cash flow relief in the short- to medium-term, and a sustained reduction of our debt service burden is necessary. This needs to be supplemented by significant balance of payments support from our international partners. We will also formulate a debt management strategy, complemented by annual borrowing plans. This will incorporate the objective of reducing the cost and risk of public debt, while taking into account monetary and fiscal policy objectives. To this end, we will seek further assistance of the Fund's Monetary and Capital Markets Department.

\section{Monetary, Exchange Rate, and Financial Sector Policies}

18. The primary objective of our monetary policy is to achieve price stability. With forecasts for continued low inflation, there appears to be room to loosen monetary policy, while ensuring that interest rates remain positive in real terms. Beginning in July we will begin a structural and measured reduction in reserve requirements and the local asset ratio, as conditions permit, to support a recovery in credit to the economy and an unwinding of the effects of longstanding financial repression. Nevertheless, we stand ready to ensure that inflationary pressures do not re-emerge.

\section{Monetary policy is based on reserve money targeting and the CBS will continue} to rely on indirect instruments of monetary management. In addition to the recently introduced weekly deposit auctions, which have become an effective liquidity management tool, we plan to set up a standing credit facility and develop operational procedures for using CBS's portfolio of treasury bills in open market operations. We have developed and intend to further improve the liquidity forecasting and reserve money programming framework, and strengthen cash flow management through improved policy coordination and information sharing between the Ministry of Finance and the CBS. Going forward, we will continue to expand policy tools, including introducing repo and reverse repo instruments, developing the interbank foreign exchange market, strengthening CBS governance, and work to elucidate the monetary transmission mechanism.

\section{Monetary measures}

\begin{tabular}{|ll|l|}
\hline \multicolumn{2}{|l|}{ Action } & Timing \\
\hline - & Make operational new lending facilities to commercial banks & June 2009 \\
\hline • & Introduce repo and reverse-repo facility & June 2009 \\
\hline - & $\begin{array}{l}\text { Reduce the local asset ratio further with the aim of phasing it out over } \\
\text { the program period as conditions permit, in consultation with IMF staff }\end{array}$ & 2009 \\
\hline - & Improve liquidity forecasting and strengthen monetary targeting & September 2009 \\
\hline • & Implement a structural and measured reduction in reserve requirements & 2009 \\
\hline
\end{tabular}


20. We will maintain the floating exchange rate regime. CBS intervention in the foreign exchange rate market will be limited to smoothing excessive volatility and ensuring orderly market conditions, subject to the achievement of our reserve money and external reserves targets. The goal is to strike a balance between the disinflation objective and buildup of international reserves, which are expected to reach 1.3 months of prospective imports by end-2009. We will continue, with IMF technical support, to introduce measures to strengthen nascent foreign exchange institutions and promote efficiency and transparency in the market.

\begin{tabular}{|ll|l|}
\hline \multicolumn{2}{|l|}{ Action } & Timing \\
\hline - & $\begin{array}{l}\text { Prepare and submit to the National Assembly a modernized, simplified } \\
\text { and transparent foreign exchange act. }\end{array}$ & June 2009 \\
\hline - & Establish a Foreign Reserves Management Committee at the CBS & June 2009 \\
\hline - & Adopt foreign reserves management and investment guidelines & September 2009 \\
\hline - & $\begin{array}{l}\text { Establish stronger controls over day-to-day foreign exchange } \\
\text { operations by separating key responsibilities and reporting lines }\end{array}$ & September 2009 \\
\hline - & $\begin{array}{l}\text { Introduce a net open position limit of 30 percent for class A bureau de } \\
\text { change }\end{array}$ & September 2009 \\
\hline - & $\begin{array}{l}\text { Introduce an augmented real-time gross settlement foreign exchange } \\
\text { system }\end{array}$ & 2010 \\
\hline
\end{tabular}

\section{Financial sector development and stability}

\section{We are committed to improving the efficiency and operations of the financial} sector. Modernizing the regulatory framework and strengthening supervisory capacity are key components of our reform agenda, drawing on IMF technical assistance. We are closely monitoring the operations and capitalization of financial institutions, which have proven resilient to large fluctuations in interest and exchange rates. Stress tests undertaken on the basis of the more stringent provisioning requirements to be introduced in June 2009 indicate that the banking sector's net tangible capitalization ratio would remain above minimum standards. Privatization of state-owned financial institutions remains a medium-term objective and we have sought technical support in this endeavor.

\begin{tabular}{|l|l|}
\hline Action & Timing \\
\hline$\bullet \quad \begin{array}{l}\text { Presentation to the National Assembly of a modernized and } \\
\text { strengthened Financial Institutions Act with IMF technical support }\end{array}$ & June 2009 \\
\hline $\begin{array}{l}\text { Further strengthen financial sector supervision through implementation of } \\
\text { the action plan developed in consultation with IMF technical experts in } \\
\text { December 2007, including: }\end{array}$ & \\
\hline$\bullet \quad \begin{array}{l}\text { Issue determination on minimum requirements for appointing external } \\
\text { auditors, which will replace existing circular }\end{array}$ & June 2009 \\
\hline
\end{tabular}




\begin{tabular}{|ll|l|}
\hline \multicolumn{2}{|l|}{ Action } & Timing \\
\hline - & $\begin{array}{l}\text { Update credit concentration regulations and connected lending } \\
\text { regulations in parallel with amendments to the Financial Institutions } \\
\text { Act }\end{array}$ & June 2009 \\
\hline - & Introduce documentation on the acquisition of substantial interests & June 2009 \\
\hline - & $\begin{array}{l}\text { Introduce manual for application to acquire substantial interest in } \\
\text { commercial banks }\end{array}$ & July 2009 \\
\hline - & Introduce manual on prompt corrective actions & September 2009 \\
\hline - & $\begin{array}{l}\text { Introduce documentation on mergers and acquisitions of assets and } \\
\text { liabilities of other financial institutions }\end{array}$ & December 2009 \\
\hline- & CBS to publish commercial banking supervision report & December 2009 \\
\hline
\end{tabular}

\section{Governance and safeguards measures}

22. We are committed to continued rapid implementation of measures to improve CBS governance, financial and accounting systems, and internal audit and control procedures, as identified in the IMF safeguards report. We are working closely with the IMF to address all of these recommendations over the next two years.

\begin{tabular}{|ll|l|}
\hline Action & Timing \\
\hline - & Complete the CBS Procedures manual & December 2009 \\
\hline - & Adopt a business continuity plan for CBS & June 2010 \\
\hline $\begin{array}{l}\text { Establish a new T-Bill allotment committee to ensure that the } \\
\text { tendering process exercise is more transparent. }\end{array}$ & June 2009 \\
\hline $\begin{array}{l}\text { Begin transition to IFRS accounting standards at CBS by 2010 } \\
\text { financial year }\end{array}$ & June 2009 \\
\hline$-\quad \begin{array}{l}\text { Launch a tender to upgrade CBS's IT system, particularly to introduce } \\
\text { an integrated accounting framework }\end{array}$ & September 2009 \\
\hline & $\begin{array}{l}\text { Set-up a procedure to systematically record all off balance sheet items } \\
\text { in the CBS in conjunction with IT system upgrade }\end{array}$ & December 2009 \\
\hline
\end{tabular}

\section{Statistics}

23. The government will strive to improve the quality, periodicity, and timeliness of macroeconomic statistics and address deficiencies in key macroeconomic data - especially GDP estimates, and external sector statistics, to improve their usefulness for economic analysis, and policy formulation. The government has requested technical assistance from the IMF Statistics Department (fiscal accounts) and from the World Bank (to strengthen the National Statistic Bureau (NSB). We will bring Seychelles into line with international best practice, by aiming to implement the IMF's Special Data Dissemination Standard (SDDS). We will set up a central coordination unit at the NSB that acts as a data-bank and a hub for data distribution, and monitors the timeliness of submission of the data by various agencies. 


\section{Program Financing}

24. For 2009, the external nonproject financing requirement is expected to be covered by disbursements from the IMF under the SBA (30 percent of quota; SDR 2.64 million; US\$3.85 million), from the joint World Bank and African Development Bank Development Policy Loan (US\$19 million) and from external debt relief (US\$83 million, including on the US\$15 million that was accumulated as arrears in the first quarter), following US\$3 million cashflow relief secured from the Paris Club creditors, and by additional program financial support, primarily from the European Union.

\section{Program Monitoring}

25. The SBA will henceforth be monitored by semi-annual program reviews. The quarterly quantitative performance criteria for 2009 (end-June and end-September) have been revised as shown in Table 1, with the end-December targets now proposed as indicative. The structural benchmarks and performance criterion are shown in Table 2. The non zero ceilings on the contracting or guaranteeing of external debt are to allow for budgeted public project finance and program support from multilateral institutions exclusively.

26. The completion of the third review under the program will be based on meeting the quantitative performance criteria (QPCs) at end-September 2009. The third review will also focus on further strengthening operations at the CBS, reinforcing public financial management, progress on normalizing relations with external creditors and implementation of the tax policy reform. We intend to request a successor arrangement under the Extended Fund Facility and cancellation of the SBA before end-December 2009. The fourth program review under the SBA would be based on meeting end-March 2010 QPCs and is scheduled to be completed by end-June 2010. Quarterly financing assurance reviews will continue as long as public debt arrears remain outstanding.

\section{The attached Technical Memorandum of Understanding (TMU) defines the} quantitative performance criteria, indicative targets and adjusters under the program. Seychelles will avoid introducing new exchange restrictions, multiple currency practices, or bilateral payments agreements in contradiction with Article VIII of the IMF's Articles of Agreement and imposing any import restrictions for balance of payments reasons. We stand ready to adopt any additional measures, in consultation with IMF staff, which may become necessary to ensure program success. 
Table 1. Seychelles: Quantitative Performance Criteria Under the Stand-By Arrangement, December 2008-December 2009 (Millions of Seychelles rupees; end-of-period)

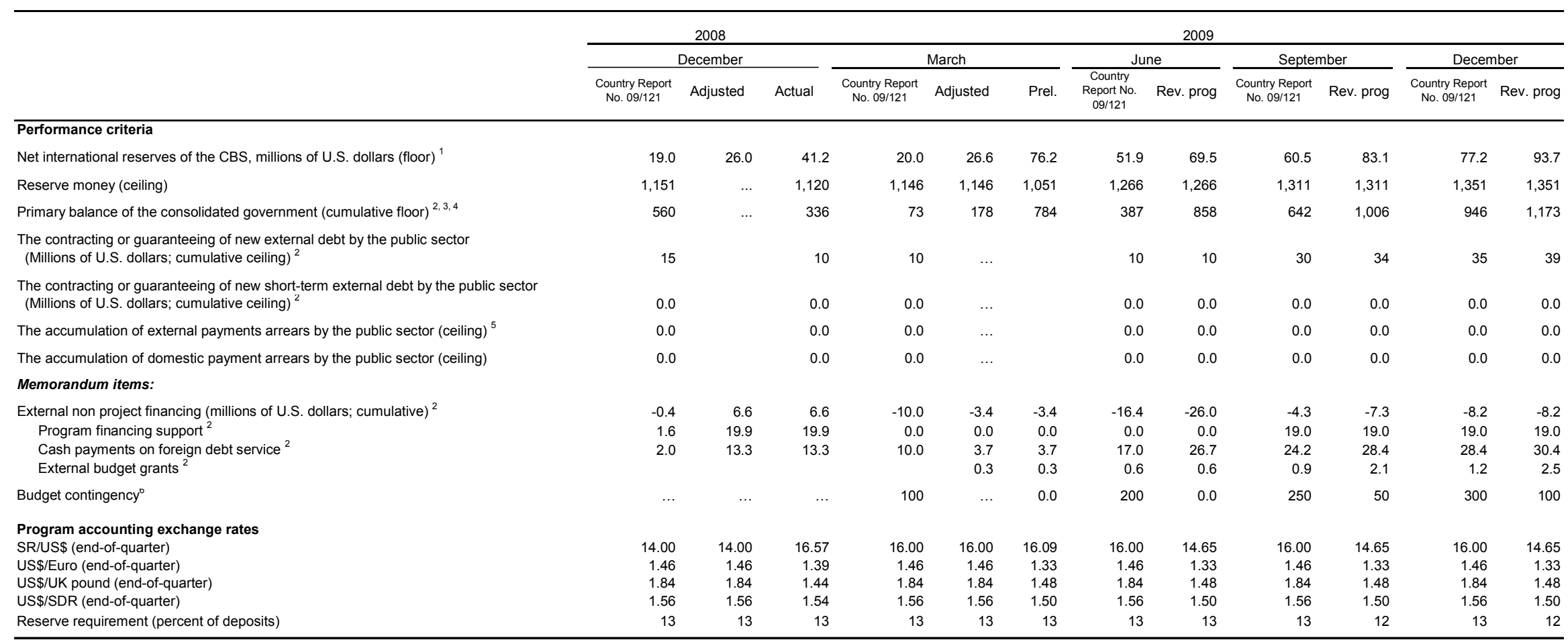

Sources: Seychelles authorities, and IMF staff estimates and projections.

1 The floor will be adjusted downwards (upwards) for any shortfall (excess) in external nonproject financial support from that assumed in the program.

Cumulative flows from the beginning of the calendar year.

The floor will be adjusted upwards for any unused amounts of the contingency in the budget for the recapitalization of the banking sector assumed in the program. Amounts of the contingency in excess

of programmed amounts need to be funded within the program limits.

The floor on the primary fiscal surplus will be adjusted upwards (downwards) by any excess (shortfall) of external budget grants from that assumed in the program.

${ }^{5}$ The non-accumulation of new external payment arrears constitutes a continuous performance criterion. Excludes arrears for which a rescheduling agreement is sough

${ }_{6}^{6}$ So external arrears were accumulated in December 2008 , but cleared before end-December.

${ }^{6}$ The floor on the primary surplus of the consolidated government will be adjusted upwards by the cumulative amount of the contingency for recapitalizing the state-controlled financial institutions which is not used for that purpose. 
Table 2. Seychelles: Structural Benchmarks and Performance Criterion, 2008-09

Measure

Target date

Status

Structural performance criterion

- Complete tax audits by Seychelles Revenue End-June 2009 Commission of the 20 largest companies

Most audits completed and remainder being finalized in June. and target expected to be met

\section{Structural benchmarks}

- Submit to the National Assembly a revision

End-April 2009 of the CBS act to strengthen governance and operations (MEFP, ๆ30).

- Promulgate updated credit classification and End-April 2009 provisioning regulations and strengthened commercial bank capitalization norms.

(MEFP, П32).

Approved by

Parliament on April

28, 2009

CBS board adopted credit classification norms and provisioning regulations on April 30. Capitalization norms were adopted on May 29, 2009

- Adopt a tax policy reform strategy with FAD End-June 2009 TA (MEFP, П20).

Strategy announced June 10, 2009

- Adopt a modernized and strengthened

End-June 2009

In progress and financial institutions act, with IMF T/A support. (MEFP, П32).

- Introduce a treasury single account (MEFP, T19).

End-

In progress

September 2009

End-

September 2009

In progress investment guidelines (MEFP, 『31).

End-September 2009

In progress

- Adopt Public Enterprise Monitoring and Control act (MEFP, \16)

End-

December 2009 I22)

End-December 2009 supervision report(MEFP, П21)

End-

- Amend the Business Tax Act in line with the tax reform strategy (MEFP, ๆ13)

December 2009 
Table 3. Seychelles: Reform Measures Implemented in January-May 2009

Action

Timing

Fiscal policy

Introduce GST on casinos

March

Remove the GST on animal feed March

Appoint a qualified financial controller to the Ministry of Health to March

strengthen expenditure control

Commence work towards implementation of a treasury single account March

Finalize annual pubic debt report March

Gather comprehensive data on parastatal debt and finances March

Hire additional qualified staff and equip the public enterprise monitoring June

unit

Set up a Financial Analysis Branch at Ministry of Finance to assess April

investment proposals.

\section{Monetary policy}

Introduce remuneration on statutory commercial bank reserves above a March

certain threshold at an interest rate that reflects the financial cost to

commercial banks

Increase required reserves maintenance period to two weeks

Reduce to 30 per cent of capital the net open positions on foreign

exchange, effective end June 2009

Establish a platform at the CBS for interbank foreign exchange trading

\section{CBS governance and safeguards}

Disclosure in 2008 Annual CBS report of off balance sheet items, March including pledged assets and earmarked or blocked funds

Submit to Parliament a revised CBS Act, inter alia to strengthen April

governance and operations.

Publish external audit letter and accounts on CBS website April

\section{Exchange regime}

With technical assistance from the IMF, establish a platform at the CBS for interbank foreign exchange trading/fixing

April

April

March

\section{Financial sector reforms}

Further strengthen financial sector supervision through implementation of the action plan developed in consultation with IMF technical experts in December 2007, including:

- Introduction of new liquidity risk management regulations

March

- Introduce new bank and bureau de change licensing regulations

March

- and update documentation requirements for banks

- Update credit classification and provisioning regulations

April

- Update and strengthen commercial bank capitalization norms 


\section{ATTACHMENT II}

\section{SEyChelles: TeChNiCAL MeMorandum OF UNDERSTANDing}

1. This technical memorandum of understanding presents the definitions of variables included in the quantitative performance criteria and indicative targets set out in the memorandum of economic and financial policies (MEFP), the key assumptions, and the reporting requirements of the Government and the Central Bank of Seychelles (CBS) needed to adequately monitor economic and financial developments. The quantitative performance criteria and indicative targets, and the structural performance criterion and benchmarks for 2008-09 are listed in Tables 1 and 2 of the MEFP, respectively.

\section{Quantitative Performance Criteria}

\section{A. Net International Reserves of the CBS (Floor)}

\section{Definition}

2. Net international reserves (NIR) of the CBS are defined for program monitoring purposes as reserve assets of the CBS, minus reserve liabilities of the CBS (including liabilities to the IMF). Reserve assets of the CBS are claims on nonresidents that are readily available (i.e., liquid and marketable assets, free of any pledges or encumberments and excluding project balances and blocked or escrow accounts, and bank reserves in foreign currency maintained for the purpose of meeting the reserve requirements), controlled by the CBS, and held for the purpose of intervening in foreign exchange markets. They include holdings of SDRs, holdings of foreign exchange, demand and short-term deposits at foreign banks abroad, fixed-term deposits abroad that can be liquidated without penalty, and any holdings of investment-grade securities. Reserve liabilities of the CBS comprise liabilities to nonresidents contracted by the CBS, any net off-balance-sheet position of the CBS (futures, forwards, swaps, or options) with either residents or nonresidents, including those to the IMF.

\section{Calculation method}

3. For program monitoring purposes, reserves assets and liabilities at each test date, must be converted into U.S. dollars using the end of period exchange rates assumed in the program.

\section{Monitoring and reporting}

4. At each program test date, the quarterly net international reserves data submitted by the CBS to the IMF will be audited by the CBS external auditors in accordance with International Standards on Auditing, to ensure conformity with the program definition and calculation methods. Reports should be submitted to the CBS, with a copy to the IMF, no later than two months after each test date. 


\section{Adjusters}

5. The floor on the CBS's NIR will be adjusted upward (downward) by the amount by which the external non-project loans and cash grants exceeds (falls short of) the amounts assumed in the program (MEFP Table 1). The floors will also be adjusted upwards (downwards) by the amount that external debt service payments fall short (exceed) the amounts assumed in the program.

\section{B. Reserve Money (Ceiling)}

\section{Definition}

6. Reserve money is equivalent to currency issued and deposits held by financial institutions at the central bank (bank reserves), including those denominated in foreign currencies. Evaluation of performance of reserve money with respect to the program ceiling will be done at the program accounting exchange rate.

\section{Monitoring and reporting}

7. For each program test date, the quarterly reserve money data submitted by the CBS to the IMF will be audited by the CBS' external auditors in accordance with International Standards on Auditing, to ensure conformity with the program definition. Reports should be submitted to the CBS, with a copy to the IMF, no later than two months after each test date.

\section{Primary Balance of the Consolidated Government (Cumulative Floor)}

8. The consolidated government primary balance from above the line on a commitment basis is defined as total consolidated government and social security fund revenues (excluding privatization and long-term lease income receipts) less all noninterest (primary) expenditures of the government and social security fund.

\section{Adjusters}

9. The floor on the primary surplus of the consolidated government will be adjusted upwards by the cumulative amount of the contingency for recapitalizing the state-controlled financial institutions which is not used for that purpose. Any amounts spent for the recapitalizing of the statecontrolled financial institutions in excess of programmed contingency will need to be funded within the program limit on the primary balance.

10. The floor on the primary surplus of the consolidated government will be adjusted upward (downward) by the amount by which external budget grants exceeds (falls short of) the amounts assumed in the program (MEFP Table 1). 


\section{Public External Debt (Ceiling)}

11. The ceiling applies to the contracting or guaranteeing of new external liabilities by the public sector (including the central government, the CBS, and all public agencies and parastatals for operations that are not directly linked to commercial activities). The ceiling does not apply to the use of Fund resources, operations related to external debt restructuring; normal import related credits; purchases of treasury securities by nonresidents; or borrowing by parastatals in the conduct of normal commercial operations. The non zero ceilings on the contracting or guaranteeing of external debt are to allow for normal public project finance and program support from multilateral institutions exclusively. Debt shall be valued in U.S. dollars at program exchange rates.

- A zero subceiling on short-term external debt applies continuously to the contracting or guaranteeing of short-term external debt by the public sector, with an original maturity of up to and including one year.

12. For the purpose of this performance criterion, the term "debt" has the meaning set forth in point No. 9 of the Executive Board's Guidelines on Performance Criteria with Respect to Foreign Debt (Decision No. 12274-(00/85)). Debt is understood to mean a current, non contingent liability, created under a contractual arrangement through the provision of value in the form of assets (including currency) or services, and which requires the obligor to make one or more payments in the form of assets (including currency) or services, at some future points in time; these payments will discharge the principal and/or interest liabilities incurred under the contract. The ceiling on contracting official and officially guaranteed external debt includes all form of debt, including:

(i) loans, i.e., advances of money to the obligor by the lender made on the basis of an undertaking that the obligor will repay the funds in the future (including deposits, bonds, debentures, commercial loans, and buyers credits) and temporary exchanges of assets that are equivalent to fully collateralized loans under which the obligor is required to repay the funds, and usually pay interest, by repurchasing the collateral from the buyer in the future (such as repurchase agreements and official swap arrangements);

(ii) suppliers credits, i.e., contracts where the supplier permits the obligor to defer payments until some time after the date on which the goods are delivered or services are provided; and,

(iii) leases, i.e., arrangements under which property is provided which the lessee has the right to use for one or more specified period(s) of time that are usually shorter than the total expected service life of the property, while the leasor retains title to the property. The debt is the present value (at the inception of the lease) of all lease payments expected to be made during the period of the agreement excluding those payments that cover the operation, repair, or maintenance of the property.

(iv) Arrears, penalties, and judicially awarded damages arising from the failure to make payment under a contractual obligation that constitutes debt are debt. 


\section{E. External Arrears of the Public Sector}

13. The nonaccumulation of arrears to external creditors will be a continuous performance criterion under the program. External payments arrears for program monitoring purposes are defined as the amount of external debt service due and not paid within the contractually agreed period, including contractual and late interest. Arrears resulting from the nonpayment of debt service for which a clearance framework has been agreed or a rescheduling agreement is sought are excluded from this definition.

\section{F. Budget Expenditure Arrears}

14. The nonaccumulation of budget expenditure arrears will be a continuous performance criterion under the program. Budget expenditure arrears are defined as the sum of (1) any invoice that has been received by a spending agency from a supplier of goods, services, and capital goods delivered and verified, and for which payment has not been made within the contractually agreed period, or in the absence of a grace period, within 30 days; (2) unpaid wages, pensions, or transfers, pending for longer than 30 days to domestic or foreign residents, irrespective of the currency denomination of the debt.

\section{DATA AND INFORMATION}

15. The Seychelles authorities (government and CBS) will provide Fund staff with the following data and information according to the schedule provided.

\section{The CBS will report}

Weekly (within one week from the end of the period)

- Reserve money.

- Foreign exchange reserves position.

- A summary table on the foreign exchange market transactions.

- The results of the liquidity deposit auctions, primary treasury bill auctions, and secondary auctions.

Monthly (within 4 weeks from the end of the month)

- The monetary survey in the standardized report form format.

- The foreign exchange cash flow, actual and updated.

- Financial soundness indicators.

- Stock of government securities in circulation by holder (banks and nonbanks) and by original maturity and the debt service profile report. 


\section{The Ministry of Finance will report}

Monthly (within 2 weeks from the end of the month):

- Consolidated government operations on a commitment basis and cash basis in the IMFsupported program format.

- The detailed revenues and expenditures of the central government and social security fund.

- Monthly accounts of the public nonbank financial institutions.

- Import and export data from the customs department.

- Monthly public debt report.

The government and CBS will consult with Fund staff on all economic and financial measures that would have an impact on program implementation, and will provide any additional relevant information as requested by Fund staff. 


\section{APPENDIX II \\ Seychelles: Debt SuStainability Analysis}

The debt sustainability analysis (DSA) confirms that public debt in Seychelles remains unsustainable even after restructuring of Paris Club (PC) debt in April 2009. The highly concessional restructuring agreement with PC creditors in mid-April 2009, which recognized Seychelles' limited payments capacity, was an important step in toward achieving sustainability. However, even with the strong and sustained fiscal adjustment assumed under the program, a further significant reduction of the external debt burden is required to restore longer-term sustainability. Against this background, engagement in good-faith discussions with all external creditors to achieve a restructuring on comparable terms is critical.

\section{Macroeconomic assumptions}

The debt sustainability analysis is based on the macroeconomic framework presented in the present staff report:

- Near-term economic growth outlook has been revised downwards on account of the global recession, with real GDP expected to shrink by about 10.7 percent in 2009 . Growth is expected to recover only gradually from 2010 onwards, supported by resumption of FDIfinanced projects and recovery of tourism, and stabilize at around 5 percent a year in the long term.

- $\quad$ Supported by tight fiscal and monetary policy, inflation has declined sharply and is expected to remain in the low single digits for the remainder of 2009 , aided by sharp declines in world food and fuel prices. It is assumed to remain at around 3 percent going forward in line with major trading partners' inflation.

- $\quad$ The near-term current account outlook has worsened from the previous analysis (done at the time of the first review) as piracy and the global economic crisis are expected to restrain exports and tourism earnings in 2009-10. The tourism forecasts for 2009 have been revised to a 22 percent drop, from the originally projected 25 percent decline, while tuna exports are expected decline by 21 percent and by a further 6 percent in 2010 . Nevertheless, over the medium-term, tourism growth is expected to recover, peaking at 10.6 percent in 2013 and moderating to a long-run steady rate of around 8 percent a year. Given its comparative advantage, tuna exports are expected to revive by 4.5 percent in 2011.

- $\quad$ Net FDI inflows are expected to decline sharply in 2009 to 27 percent of GDP, as a number of large hotel projects have been postponed or slowed. This reflects tighter credit conditions and lower near-term outlook for tourism inflows. FDI flows are expected to recover from 2010 and stabilize at 26 percent of GDP over the long-run.

- $\quad$ Official foreign exchange reserves are targeted to build-up gradually to about 3.8 months of imports by 2019, a necessary recovery from the depleted levels of 2008. 


\section{Evolution of the debt stock and debt burden}

- $\quad$ Public external debt is estimated at US\$802.2 million (87.1 percent of GDP) at end-2008 (Table 1). About 60 percent of the debt was owed to commercial creditors, while the PC countries account for about 19 percent of the total. Seychelles has no arrears with multilateral creditors. At end-2008 some 42 percent of the debt stock was in arrears (US\$333.5 million).

- In April 2009, PC creditors granted exceptional treatment to Seychelles under the Evian approach. ${ }^{1}$ US\$140 million of arrears were normalized (including South Africa). The restructuring of Seychelles debt will involve both flow and stock rescheduling with the debt stock reduced by 45 percent in two tranches and the remaining amounts rescheduled over 18 years including a five-year grace. No repayments will be made in 2009 other than a "goodwill" payment of US\$1 million by end-June, with very limited payments due in the next few years as moratorium interest is partially deferred.

- $\quad$ Despite the PC debt restructuring, the stock of public external debt is expected to rise to 108 percent of GDP by end 2009, primarily reflecting disbursements from multilateral agencies and the depreciation of the rupee.

\section{Baseline Scenario}

The PC agreement leads to a significant reduction in the debt ratios but without further restructuring from other creditors on a comparable basis public debt remains unsustainable (Tables 2, 2a). However, the PC agreement is not tenable on its own, since a formal requirement for the effectiveness of the agreement is securing comparability of treatment from other creditors. This scenario assumes that the non-restructured debt (including arrears) is serviced and that the financing gaps are closed, as assumed in earlier analyses, by a flow rescheduling on market terms at LIBOR plus 1300 basis points and with a three-year maturity. ${ }^{2}$ Over the next ten years, the public external debt ratio would rise steadily to around 326 percent of GDP (310.5 percent of exports) in 2019. Furthermore, the bound tests highlight that the external debt is extremely sensitive to the underlying assumptions, with even moderate shocks triggering dramatic explosive dynamics (Figure 1a). Notwithstanding the strong fiscal adjustment (Table 2b and Figure 1b), the analysis indicates a similar unsustainable profile for overall public sector debt over the medium run, at 342.7 percent of GDP, driven by dynamics of external debt.

\footnotetext{
${ }^{1}$ The agreement covered all the debt to Paris Club. South Africa, a significant non-Paris Club creditor, participated in the meeting and was a signatory of the minute.

${ }^{2}$ The assumed risk premium is designed to mimic the current level of EMBI spreads on sovereign external debt of other highly indebted countries with significant vulnerabilities, for which trading of debt securities is more active.
} 


\section{Alternative Scenario $^{3}$}

Under this scenario, public external debt at end-2008 is restructured on comparable cash flow terms with the PC agreement (the agreement with PC creditors requires Seychelles to seek promptly from all other external commercial and bilateral creditors debt restructuring on comparable terms). Public external debt would decline steadily to 60.4 percent in 2014 and remain under 60 percent through 2019 (Table 3a and Figure 2a). Total public debt (including domestic debt) would decline to 62.5 percent of GDP by 2019 (Table 3b, Figure 2b). However, assuming key variables are at their historical averages the external debt ratio would rise steadily to 104.9 percent of GDP, pointing to the vulnerability of the outlook.

Standardized bound tests show that Seychelles's public debt is particularly exposed to current account shocks and interest rate shocks. Assuming a half standard deviation shocks to the current account (8.3 percent increase in the deficit), the debt stock would increase to 493.2 percent (baseline scenario) in 2019 and 118.7 percent (alternative scenario).

Table 1. External Debt ${ }^{1}$

\begin{tabular}{lr}
\hline & End-2008 \\
\hline External debt stock ${ }^{2}$ & 802.2 \\
Multilateral & 57.0 \\
Bilateral & 267.4 \\
Paris Club & 151.7 \\
Non-Paris Club & 115.8 \\
Commercial & 477.7 \\
& \\
External debt arrears & 333.5 \\
Multilateral & 0.0 \\
Bilateral & 184.5 \\
Paris Club & 124.2 \\
Non-Paris Club & 60.4 \\
Commercial & 149.0 \\
\hline${ }^{1}$ Preliminary and subject to reconciliation with creditors. Includes CB \\
${ }^{2}$ Includes arrears.
\end{tabular}

\footnotetext{
${ }^{3}$ This hypothetical scenario is included for illustrative purposes only. It is based on technical assumptions regarding the restructuring of debts to all other non-Paris Club creditors on terms comparable to those agreed with Paris Club creditors, without pre-judging the terms of such a restructuring.
} 
Table 2. Seychelles: Balance of Payments Baseline Scenario (Assuming Paris Club Restructuring), 2007-19 (Millions of U.S. dollars)

\begin{tabular}{|c|c|c|c|c|c|c|c|c|c|c|c|c|c|}
\hline & 2007 & 2008 & 2009 & 2010 & 2011 & 2012 & 2013 & 2014 & 2015 & 2016 & 2017 & 2018 & 2019 \\
\hline & & Prel. & Proj. & Proj. & & & & & & & & & \\
\hline $\begin{array}{l}\text { Current account } \\
\text { (Percent of GDP) }\end{array}$ & $\begin{array}{r}-213 \\
-21\end{array}$ & $\begin{array}{r}-420 \\
-46\end{array}$ & $\begin{array}{r}-250 \\
-37\end{array}$ & $\begin{array}{r}-343 \\
-45\end{array}$ & $\begin{array}{r}-422 \\
-51\end{array}$ & $\begin{array}{r}-445 \\
-48\end{array}$ & $\begin{array}{r}-500 \\
-50\end{array}$ & $\begin{array}{r}-578 \\
-53\end{array}$ & $\begin{array}{r}-659 \\
-56\end{array}$ & $\begin{array}{r}-772 \\
-61\end{array}$ & $\begin{array}{r}-911 \\
-66\end{array}$ & $\begin{array}{r}-1,082 \\
-73\end{array}$ & $\begin{array}{r}-1,283 \\
-80\end{array}$ \\
\hline Trade balance & -175 & -386 & -146 & -187 & -172 & -142 & -127 & -119 & -104 & -100 & -97 & -97 & -92 \\
\hline $\begin{array}{l}\text { Income, net } \\
\text { Of which: interest payments due }{ }^{1}\end{array}$ & $\begin{array}{l}-72 \\
-33\end{array}$ & $\begin{array}{r}-103 \\
-33\end{array}$ & $\begin{array}{r}-155 \\
-92\end{array}$ & $\begin{array}{l}-215 \\
-124\end{array}$ & $\begin{array}{l}-311 \\
-202\end{array}$ & $\begin{array}{l}-363 \\
-236\end{array}$ & $\begin{array}{l}-435 \\
-290\end{array}$ & $\begin{array}{l}-523 \\
-357\end{array}$ & $\begin{array}{l}-620 \\
-432\end{array}$ & $\begin{array}{l}-739 \\
-525\end{array}$ & $\begin{array}{l}-882 \\
-642\end{array}$ & $\begin{array}{r}-1,054 \\
-786\end{array}$ & $\begin{array}{r}-1,262 \\
-962\end{array}$ \\
\hline Current transfers, net & 33 & 69 & 52 & 60 & 60 & 61 & 62 & 63 & 65 & 66 & 68 & 70 & 71 \\
\hline $\begin{array}{l}\text { Capital and financial account } \\
\text { Capital account }\end{array}$ & $\begin{array}{r}261 \\
8\end{array}$ & $\begin{array}{r}172 \\
5\end{array}$ & $\begin{array}{r}483 \\
37\end{array}$ & $\begin{array}{r}380 \\
37\end{array}$ & $\begin{array}{r}472 \\
5\end{array}$ & $\begin{array}{r}500 \\
5\end{array}$ & $\begin{array}{r}561 \\
5\end{array}$ & $\begin{array}{r}634 \\
6\end{array}$ & $\begin{array}{r}711 \\
6\end{array}$ & $\begin{array}{r}822 \\
6\end{array}$ & $\begin{array}{r}961 \\
6\end{array}$ & $\begin{array}{r}1,132 \\
6\end{array}$ & $\begin{array}{r}1,333 \\
6\end{array}$ \\
\hline Financial account & 254 & 167 & 446 & 342 & 467 & 495 & 555 & 628 & 705 & 816 & 955 & 1,126 & 1,327 \\
\hline Direct investment, net & 225 & 346 & 183 & 213 & 236 & 239 & 258 & 289 & 301 & 326 & 352 & 381 & 411 \\
\hline Portfolio investment, net & 31 & 1 & 0 & 0 & 0 & 0 & 0 & 0 & 0 & 0 & 0 & 0 & \\
\hline Other investment, net & -2 & -180 & 263 & 129 & 232 & 257 & 297 & 339 & 403 & 490 & 602 & 745 & 915 \\
\hline Net errors and omissions & -8 & 101 & 23 & 0 & 0 & 0 & 0 & 0 & 0 & 0 & 0 & 0 & \\
\hline Overall balance & 40 & -147 & 257 & 37 & 50 & 56 & 60 & 56 & 51 & 50 & 50 & 50 & 50 \\
\hline Financing & -40 & 147 & -257 & -37 & -50 & -56 & -60 & -56 & -51 & -50 & -50 & -50 & -50 \\
\hline Change in net international reserves (increase: - ) & 49 & -27 & -54 & -37 & -50 & -56 & -60 & -56 & -51 & -50 & -50 & -50 & $\begin{array}{l}-50 \\
-50\end{array}$ \\
\hline Change in gross official reserves (increase: -) & 49 & -36 & -58 & -50 & -50 & -50 & -50 & -50 & -50 & -50 & -50 & -50 & -50 \\
\hline Prospective liabilities to IMF, net & 0 & 10 & 4 & 13 & 0 & -6 & -10 & -6 & -1 & 0 & 0 & 0 & \\
\hline Other NFA (increase: - ) & 0 & 0 & -10 & 0 & 0 & 0 & 0 & 0 & 0 & 0 & 0 & 0 & \\
\hline Exceptional financing & -89 & 174 & -193 & 0 & 0 & 0 & 0 & 0 & 0 & 0 & 0 & 0 & \\
\hline Change in arrears (increase: +$)^{3,4}$ & -89 & 174 & -333 & 0 & 0 & 0 & 0 & 0 & 0 & 0 & 0 & 0 & \\
\hline Clearance of arrears & & $\ldots$ & 140 & 0 & 0 & 0 & 0 & 0 & 0 & 0 & 0 & 0 & \\
\hline Financing gap ${ }^{5}$ & 0 & 0 & 0 & 0 & 0 & 0 & 0 & 0 & 0 & 0 & 0 & 0 & \\
\hline Financing gap (before closing) & 0.0 & 0.0 & 337 & 273 & 638 & 638 & 783 & 1,003 & 1,192 & 1,466 & 1,810 & 2,224 & 2,740 \\
\hline \multicolumn{14}{|l|}{ Memorandum items: } \\
\hline Gross international reserves (stock, e.o.p.) ${ }^{6}$ & 10 & 51 & 108 & 158 & 208 & 258 & 308 & 358 & 408 & 458 & 508 & 558 & 608 \\
\hline Months of prospective imports of goods and services & 0.1 & 1 & 1 & 1.8 & 2.3 & 2.6 & 3.0 & 3.2 & 3.4 & 3.6 & 3.7 & 3.8 & 3.8 \\
\hline Public external debt $1,4,6$ & 710 & 802 & 885 & 1,008 & 1,229 & 1,477 & 1,766 & 2,088 & 2,486 & 2,971 & 3,572 & 4,318 & 5,233 \\
\hline (Percent of GDP) & 69.2 & 87 & 130 & 132.3 & 147.5 & 161.0 & 175.1 & 191.7 & 211.1 & 233.6 & 259.9 & 290.8 & 326.2 \\
\hline Of which: arrears ${ }^{13,4}$ & 160 & 333 & 0 & 0 & 0 & 0 & 0 & 0 & 0 & 0 & 0 & 0 & \\
\hline (Percent of GDP) & 15.6 & 36 & 0 & 0 & 0 & 0 & 0 & 0 & 0 & 0 & 0 & 0 & \\
\hline GDP & 1,026 & 921 & 679 & 762 & 833 & 918 & 1,009 & 1,090 & 1,177 & 1,272 & 1,374 & 1,485 & 1,604 \\
\hline
\end{tabular}

${ }^{1}$ Projections are based on identified new borrowing and rescheduled Paris Club debt. It assumes comparable cash flow treatment for one bilateral creditor that also signed the agreement, and no debt restructuring for the remaining creditors. Arrears restructuring leads to higher rescheduled interst payments.

${ }^{2}$ Based on Paris Club agreement, the payments of principal and most interest during 2008 and through June 2009 are deferred.

${ }^{3}$ In 2008, includes accelerated amortizing notes.

${ }_{4}^{4}$ Debt forgivenesss reflects: (i) the first stage of debt reduction (about 22.5) percent in July 2009; (ii) the second stage reduction (about 22.5 percent) in July 2010 on Paris Club debt and one bilateral creditor. Assumes debt

reduction comparable to the Paris Club agreement on
${ }^{5}$ Financing gaps are assumed to be closed by a hypothetical 3-year maturity amortizing loan at libor plus 13 percent.

${ }^{6}$ Starting with 2007, refers to CBS gross international reserves net of blocked deposits and project accounts. 


\begin{tabular}{|c|c|c|c|c|c|c|c|c|c|c|c|c|}
\hline & \multicolumn{11}{|c|}{ Projections } & \multirow[b]{2}{*}{2019} \\
\hline & 2008 & 2009 & 2010 & 2011 & 2012 & 2013 & 2014 & 2015 & 2016 & 2017 & 2018 & \\
\hline \multicolumn{13}{|c|}{ I. Baseline Projections } \\
\hline External debt ${ }^{1}$ & 87.1 & 130.4 & 132.3 & 147.5 & 161.0 & 175.1 & 191.7 & 211.1 & 233.6 & 259.9 & 290.8 & 326.2 \\
\hline Change in external debt & 17.9 & 43.4 & 1.8 & 15.2 & 13.5 & 14.0 & 16.6 & 19.5 & 22.5 & 26.3 & 30.9 & 35.5 \\
\hline Identified external debt-creating flows $(4+8+9)$ & 15.9 & 12.9 & 2.7 & 11.1 & 8.9 & 9.5 & 13.5 & 16.1 & 19.3 & 23.2 & 27.9 & 32.7 \\
\hline Current account deficit, excluding interest payments & 43.4 & -5.4 & 28.5 & 26.2 & 22.6 & 20.8 & 20.3 & 19.4 & 19.4 & 19.6 & 19.9 & 20.0 \\
\hline Deficit in balance of goods and services & 41.9 & -9.2 & 24.5 & 20.6 & 15.5 & 12.6 & 10.9 & 8.8 & 7.8 & 7.0 & 6.5 & 5.8 \\
\hline Exports & 109.3 & 139.5 & 106.2 & 105.4 & 104.4 & 103.5 & 104.0 & 104.6 & 104.7 & 104.8 & 104.6 & 105.1 \\
\hline Imports & 151.1 & 130.4 & 130.8 & 126.0 & 119.9 & 116.1 & 114.9 & 113.5 & 112.6 & 111.9 & 111.2 & 110.8 \\
\hline Net non-debt creating capital inflows (negative) & -37.6 & -26.9 & -28.0 & -28.3 & -26.0 & -25.6 & -26.5 & -25.6 & -25.6 & -25.6 & -25.6 & -25.6 \\
\hline Automatic debt dynamics $^{2}$ & 10.1 & 45.2 & 2.1 & 13.1 & 12.3 & 14.2 & 19.8 & 22.4 & 25.6 & 29.3 & 33.6 & 38.3 \\
\hline Contribution from nominal interest rate & 2.2 & 14.0 & 16.5 & 24.4 & 25.8 & 28.8 & 32.8 & 36.7 & 41.3 & 46.7 & 52.9 & 60.0 \\
\hline Contribution from real GDP growth & 0.7 & 12.6 & -4.1 & -6.0 & -6.7 & -7.3 & -8.1 & -8.9 & -9.8 & -10.8 & -12.0 & -13.5 \\
\hline Contribution from price and exchange rate changes ${ }^{3}$ & 7.2 & 18.5 & -10.2 & -5.3 & -6.8 & -7.2 & -4.9 & -5.4 & -5.9 & -6.6 & -7.3 & -8.2 \\
\hline Residual, incl. change in gross foreign assets (2-3) & 2.0 & 30.5 & -0.8 & 4.2 & 4.6 & 4.5 & 3.1 & 3.3 & 3.1 & 3.1 & 3.0 & 2.8 \\
\hline External debt-to-exports ratio (in percent) & 79.7 & 93.5 & 124.5 & 139.9 & 154.2 & 169.1 & 184.3 & 201.8 & 223.0 & 248.0 & 277.9 & 310.5 \\
\hline Gross external financing need (in billions of US dollars) ${ }^{4}$ & 0.6 & 0.1 & 0.5 & 0.9 & 0.9 & 1.0 & 1.3 & 1.5 & 1.8 & 2.1 & 2.6 & 3.1 \\
\hline in percent of GDP & 66.1 & 20.5 & 67.1 & 105.8 & 94.9 & 101.8 & 117.0 & 125.2 & 139.3 & 155.7 & 173.8 & 195.0 \\
\hline \multicolumn{13}{|l|}{ Key macroeconomic assumptions } \\
\hline Nominal GDP (US dollars) & 0.9 & 0.7 & 0.8 & 0.8 & 0.9 & 1.0 & 1.1 & 1.2 & 1.3 & 1.4 & 1.5 & 1.6 \\
\hline Real GDP growth (percent) & -0.9 & -10.7 & 3.5 & 5.0 & 5.0 & 5.0 & 5.0 & 5.0 & 5.0 & 5.0 & 5.0 & 5.0 \\
\hline Exchange rate appreciation (US dollar value of local currency, change in percent) & -29.5 & -37.3 & 4.3 & 1.7 & 1.7 & 1.7 & 0.0 & 0.0 & 0.0 & 0.0 & 0.0 & 0.0 \\
\hline GDP deflator in US dollars (change in percent) & -9.4 & -17.6 & 8.5 & 4.1 & 4.9 & 4.7 & 2.9 & 2.9 & 2.9 & 2.9 & 2.9 & 2.9 \\
\hline Nominal external interest rate (percent) & 2.9 & 11.8 & 14.2 & 20.2 & 19.3 & 19.6 & 20.2 & 20.7 & 21.1 & 21.6 & 22.0 & 22.3 \\
\hline Growth of exports (US dollar terms, percent) & 15.3 & -6.0 & -14.5 & 8.5 & 9.1 & 9.0 & 8.5 & 8.7 & 8.2 & 8.1 & 7.8 & 8.5 \\
\hline Growth of imports (US dollar terms, percent) & 32.9 & -36.5 & 12.7 & 5.4 & 4.8 & 6.4 & 6.9 & 6.7 & 7.2 & 7.4 & 7.4 & 7.7 \\
\hline Current account balance, excluding interest payments & -43.4 & 5.4 & -28.5 & -26.2 & -22.6 & -20.8 & -20.3 & -19.4 & -19.4 & -19.6 & -19.9 & -20.0 \\
\hline Net non-debt creating capital inflows & 37.6 & 26.9 & 28.0 & 28.3 & 26.0 & 25.6 & 26.5 & 25.6 & 25.6 & 25.6 & 25.6 & 25.6 \\
\hline \multicolumn{13}{|c|}{ II. Stress Tests for External Debt Ratio } \\
\hline A. Alternative scenarios & & & & & & & & & & & & \\
\hline A1. Key variables are at their historical averages in $2008-13^{5}$ & 87.1 & 108.1 & 107.1 & 109.6 & 112.4 & 115.1 & 116.5 & 118.1 & 119.5 & 120.8 & 121.9 & 122.8 \\
\hline \multicolumn{13}{|l|}{ B. Bound tests } \\
\hline B1. Nominal interest rate is at baseline plus one standard deviation & 87.1 & 133.6 & 138.7 & 157.9 & 176.2 & 195.8 & 219.6 & 247.8 & 280.9 & 320.1 & 366.7 & 421.2 \\
\hline B2. Real GDP growth is at baseline minus two standard deviations & 87.1 & 135.8 & 140.2 & 158.7 & 175.7 & 193.5 & 214.0 & 238.2 & 265.9 & 298.3 & 336.3 & 380.3 \\
\hline B3. Non-interest current account is at baseline minus one standard deviations & 87.1 & 138.8 & 149.1 & 174.3 & 198.4 & 224.0 & 254.5 & 289.6 & 329.9 & 376.7 & 431.0 & 493.2 \\
\hline B4. Combination of B1-B3 using 1/4 standard deviation shocks & 87.1 & 134.0 & 139.0 & 158.2 & 176.0 & 194.8 & 217.2 & 243.4 & 273.8 & 309.3 & 351.0 & 399.2 \\
\hline B5. One time 15 percent of additional real depreciation in 2009 & 87.1 & 153.6 & 150.9 & 163.9 & 175.1 & 186.8 & 200.7 & 217.5 & 236.9 & 259.7 & 286.7 & 317.7 \\
\hline
\end{tabular}

Source: Seychelles authorities; and IMF staff projections.

Includes CBS external debt.

${ }^{2}$ Derived as $[\mathrm{r}-\mathrm{g}-\rho(1+\mathrm{g})+\varepsilon \alpha(1+\mathrm{r})](1+\mathrm{g}+\rho+\mathrm{g} \rho)$ times previous period debt stock, with $\mathrm{r}=$ nominal effective interest rate on external debt; $\rho=$ change in domestic GDP deflator in US dollar terms,

$\mathrm{g}=$ real GDP growth rate, $\mathrm{e}=$ nominal appreciation (increase in dollar value of domestic currency), and $\mathrm{a}=$ share of domestic-currency denominated debt in total external debt.

${ }^{3}$ The contribution from price and exchange rate changes is defined as $[-\rho(1+\mathrm{g})+\varepsilon \alpha(1+\mathrm{r})] /(1+\mathrm{g}+\rho+\mathrm{g} \rho)$ times previous period debt stock. $\rho$ increases with an appreciating domestic currency $(\varepsilon>0)$ and rising inflation (based on GDP deflator).

${ }^{4}$ Defined as current account deficit, plus amortization on medium- and long-term debt, plus short-term debt at end of previous period.

${ }^{5}$ The key variables include real GDP growth; nominal interest rate; dollar deflator growth; and both non-interest current account and non-debt inflows in percent of GDP. 
Figure 1a. External DSA: Bound Tests for Baseline Scenario (PC Restructuring and Nonconcessional Refinancing) ${ }^{1}$ (External debt in percent of GDP)
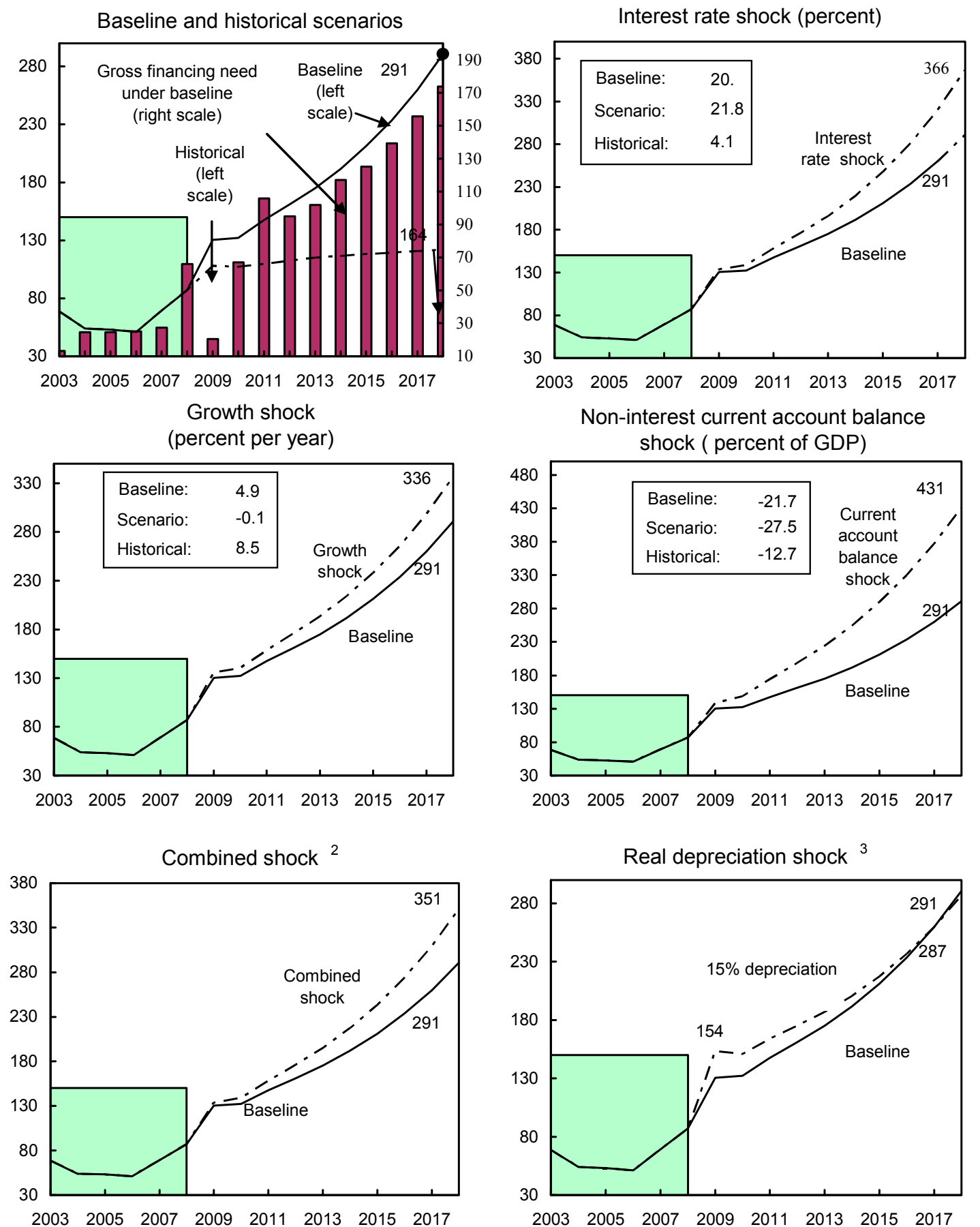

Sources: International Monetary Fund, Country desk data, and staff estimates.

${ }^{1}$ External financing gap is assumed to be closed by external borrowing at LIBOR plus $1300 \mathrm{bps}$, with three year maturity. Shaded areas represent actual data. Individual shocks are permanent one standard deviation shocks (two standard deviations for growth shock). Figures in the boxes represent average projections for the respective variables in the baseline and scenario being presented. Ten-year historical average for the variable is also shown.

${ }^{2}$ Permanent $1 / 4$ standard deviation shocks applied to real interest rate, growth rate, and current account balance.

${ }^{3}$ One-time real depreciation of additional 15 percent occurs in 2009. 
Table 2b. Seychelles: Public Sector Debt Sustainability Framework for Baseline Scenario

(PC Restructuring only and Nonconcessional Refinancing of Private Sector Creditors), 2006-19 (Percent of GDP, unless otherwise indicated)

\begin{tabular}{|c|c|c|c|c|c|c|c|c|c|c|c|c|c|c|}
\hline & \multicolumn{3}{|c|}{ Actual } & \multicolumn{10}{|c|}{ Projections } & \multirow[b]{2}{*}{2019} \\
\hline & 2006 & 2007 & 2008 & 2009 & 2010 & 2011 & 2012 & 2013 & 2014 & 2015 & 2016 & 2017 & 2018 & \\
\hline Public sector debt $^{1}$ & 132.4 & 129.8 & 139.4 & 172.2 & 162.8 & 173.8 & 183.8 & 195.4 & 210.8 & 229.5 & 252.2 & 278.7 & 309.3 & 342.7 \\
\hline $\mathrm{o} / \mathrm{w}$ foreign-currency denominated & 51.0 & 63.9 & 87.1 & 130.4 & 132.3 & 147.5 & 161.0 & 175.1 & 191.7 & 211.1 & 233.6 & 259.9 & 290.8 & 326.2 \\
\hline Change in public sector debt & -9.4 & -2.6 & 9.6 & 32.8 & -9.4 & 11.0 & 9.9 & 11.6 & 15.4 & 18.7 & 22.7 & 26.5 & 30.6 & 33.4 \\
\hline Identified debt-creating flows $(4+7+12)$ & -7.5 & -7.7 & -5.5 & 25.7 & -6.9 & 7.0 & 6.1 & 8.1 & 14.3 & 17.1 & 21.2 & 24.9 & 29.2 & 33.9 \\
\hline Primary deficit (negative means surplus) & 0.5 & 2.0 & -3.9 & -11.4 & -9.8 & -5.4 & -4.8 & -4.9 & -4.2 & -4.1 & -3.3 & -3.3 & -3.3 & -3.4 \\
\hline Revenue and grants & 41.2 & 32.2 & 36.4 & 35.7 & 34.3 & 33.6 & 33.7 & 34.3 & 34.1 & 34.9 & 34.4 & 34.7 & 34.7 & 34.8 \\
\hline Primary (noninterest) expenditure & 41.7 & 34.2 & 32.5 & 24.3 & 24.5 & 28.2 & 29.0 & 29.4 & 30.0 & 30.7 & 31.2 & 31.4 & 31.4 & 31.4 \\
\hline Automatic debt dynamics $2 /$ & -9.3 & -7.8 & 1.3 & 40.9 & 4.7 & 13.4 & 11.7 & 13.8 & 19.3 & 22.0 & 25.2 & 28.9 & 33.2 & 37.9 \\
\hline Contribution from interest rate/growth differential ${ }^{3}$ & -9.4 & -17.4 & -20.6 & -8.2 & 10.4 & 15.9 & 14.5 & 16.8 & 19.3 & 22.0 & 25.2 & 28.9 & 33.2 & 37.9 \\
\hline Of which contribution from real interest rate & 2.4 & -6.9 & -21.5 & -20.9 & 16.0 & 23.4 & 22.5 & 25.3 & 28.4 & 31.8 & 35.8 & 40.6 & 46.1 & 52.2 \\
\hline Of which contribution from real GDP growth & -11.8 & -10.5 & 0.9 & 12.7 & -5.6 & -7.6 & -8.0 & -8.5 & -9.0 & -9.8 & -10.6 & -11.7 & -12.9 & -14.3 \\
\hline Contribution from exchange rate depreciation ${ }^{4}$ & 0.1 & 9.6 & 21.9 & 49.1 & -5.7 & -2.4 & -2.7 & -3.0 & 0.0 & 0.0 & 0.0 & 0.0 & 0.0 & 0.0 \\
\hline Denominator $=1+g+p+g p$ & 1.1 & 1.2 & 1.3 & 1.2 & 1.1 & 1.1 & 1.1 & 1.1 & 1.1 & 1.1 & 1.1 & 1.1 & 1.1 & 1.1 \\
\hline Other identified debt-creating flows & 1.2 & -2.0 & -2.9 & -3.8 & -1.8 & -0.9 & -0.9 & -0.9 & -0.8 & -0.8 & -0.7 & -0.7 & -0.7 & -0.7 \\
\hline Privatization receipts (negative) & -3.6 & -2.0 & -2.9 & -3.8 & -1.8 & -0.9 & -0.9 & -0.9 & -0.8 & -0.8 & -0.7 & -0.7 & -0.7 & -0.7 \\
\hline Recognition of implicit or contingent liabilities & 4.8 & 0.0 & 0.0 & 0.0 & 0.0 & 0.0 & 0.0 & 0.0 & 0.0 & 0.0 & 0.0 & 0.0 & 0.0 & 0.0 \\
\hline Other (specify, e.g. bank recapitalization) & 0.0 & 0.0 & 0.0 & 0.0 & 0.0 & 0.0 & 0.0 & 0.0 & 0.0 & 0.0 & 0.0 & 0.0 & 0.0 & 0.0 \\
\hline Residual, including asset changes $(2-3)$ & -1.9 & 5.1 & 15.1 & 7.1 & -2.5 & 3.9 & 3.9 & 3.5 & 1.1 & 1.6 & 1.5 & 1.6 & 1.4 & -0.5 \\
\hline Public sector debt-to-revenue ratio ${ }^{1}$ & 321.6 & 403.1 & 383.0 & 482.3 & 475.0 & 517.3 & 544.6 & 569.7 & 617.4 & 658.1 & 732.0 & 802.7 & 891.6 & 985.7 \\
\hline Gross financing need ${ }^{5}$ & 53.1 & 39.4 & 45.1 & 35.8 & 63.8 & 101.0 & 91.8 & 97.6 & 106.5 & 109.9 & 122.5 & 136.8 & 151.9 & 198.6 \\
\hline in billions of U.S. dollars & 0.5 & 0.4 & 0.4 & 0.2 & 0.5 & 0.8 & 0.8 & 1.0 & 1.2 & 1.3 & 1.6 & 1.9 & 2.3 & 3.0 \\
\hline \multicolumn{15}{|l|}{ Key macroeconomic and fiscal assumptions } \\
\hline Real GDP growth (in percent) & 9.3 & 9.7 & -0.9 & -10.7 & 3.5 & 5.0 & 5.0 & 5.0 & 5.0 & 5.0 & 5.0 & 5.0 & 5.0 & 5.0 \\
\hline Average nominal interest rate on public debt (in percent) ${ }^{6}$ & 4.2 & 6.1 & 7.1 & 10.5 & 14.2 & 18.0 & 17.3 & 17.9 & 18.7 & 19.3 & 19.9 & 20.4 & 20.9 & 21.3 \\
\hline Average nominal interest rate on forex debt (in percent) ${ }^{6}$ & 2.6 & 7.5 & 4.4 & 11.4 & 13.7 & 20.1 & 19.1 & 19.5 & 20.1 & 20.6 & 21.0 & 21.5 & 21.9 & 22.2 \\
\hline Average real interest rate (nominal rate minus change in GDP deflator, in percent) & 2.1 & -5.3 & -21.3 & -20.9 & 10.2 & 15.6 & 14.2 & 15.0 & 15.8 & 16.4 & 17.0 & 17.5 & 18.0 & 18.4 \\
\hline Nominal depreciation of local currency (LC per dollar) & 0.4 & 21.4 & 41.8 & 59.4 & -4.2 & -1.7 & -1.7 & -1.7 & 0.0 & 0.0 & 0.0 & 0.0 & 0.0 & 0.0 \\
\hline Nominal appreciation (increase in US dollar value of local currency, in percent) & -0.4 & -17.6 & -29.5 & -37.3 & 4.3 & 1.7 & 1.7 & 1.7 & 0.0 & 0.0 & 0.0 & 0.0 & 0.0 & 0.0 \\
\hline Inflation rate (GDP deflator, in percent) & 2.1 & 11.4 & 28.4 & 31.4 & 4.0 & 2.4 & 3.1 & 2.9 & 2.9 & 2.9 & 2.9 & 2.9 & 2.9 & 2.9 \\
\hline Growth of real primary spending (deflated by GDP deflator, in percent) & 36.0 & -10.0 & -5.8 & -33.3 & 4.4 & 20.6 & 8.1 & 6.7 & 6.8 & 7.8 & 6.5 & 5.8 & 5.0 & 5.0 \\
\hline Primary deficit & 0.5 & 2.0 & -3.9 & -11.4 & -9.8 & -5.4 & -4.8 & -4.9 & -4.2 & -4.1 & -3.3 & -3.3 & -3.3 & -3.4 \\
\hline \multicolumn{15}{|l|}{ A. Alternative scenarios } \\
\hline A1. Key variables are at their historical averages in $2008-12^{7}$ & & & 139.4 & 187.4 & 183.7 & 190.5 & 197.4 & 204.1 & 211.5 & 219.8 & 228.3 & 237.4 & 246.6 & 254.2 \\
\hline A2. No policy change (constant primary balance) in $2008-12$ & & & 139.4 & 185.6 & 188.4 & 209.0 & 228.2 & 250.0 & 277.1 & 308.8 & 345.5 & 388.1 & 437.0 & 491.4 \\
\hline \multicolumn{15}{|l|}{ B. Bound tests } \\
\hline B1. Real interest rate is at baseline plus one-half standard deviations & & & 134.9 & 171.9 & 167.9 & 184.7 & 201.3 & 220.6 & 245.7 & 275.9 & 312.6 & 356.2 & 407.6 & 470.2 \\
\hline B2. Real GDP growth is at baseline minus two standard deviation & & & 139.4 & 180.3 & 178.9 & 201.1 & 224.7 & 253.3 & 290.5 & 336.4 & 393.0 & 461.5 & 543.6 & 629.8 \\
\hline B3. Primary balance is at baseline minus one standard deviation & & & 139.4 & 177.1 & 172.9 & 189.6 & 205.6 & 223.8 & 247.0 & 274.4 & 307.0 & 344.7 & 388.2 & 436.8 \\
\hline B4. Combination of B1-B3 using one-quarter standard deviation shocks & & & 139.4 & 184.3 & 186.1 & 211.1 & 236.9 & 267.5 & 306.5 & 354.1 & 412.1 & 482.2 & 566.2 & 665.4 \\
\hline B5. One time 15 percent additional real depreciation in $2009^{8}$ & & & 134.9 & 206.4 & 198.0 & 211.9 & 224.4 & 239.1 & 258.8 & 282.5 & 311.0 & 344.2 & 382.6 & 429.1 \\
\hline B6. 10 percent of GDP increase in other debt-creating flows in 2009 & & & 139.4 & 182.2 & 173.1 & 184.9 & 195.6 & 208.2 & 224.8 & 245.0 & 269.3 & 297.8 & 330.7 & 367.4 \\
\hline
\end{tabular}

Source: Seychelles authorities; and IMF staff projections.

${ }^{1}$ Public sector covers non-finacial public sector and the IMF loan to the CBS. Debt is on a gross basis. External and domestic financing gaps are assumed to be closed by additional borrowing.

${ }^{2}$ Derived as $[(r-p(1+g)-g+a e(1+r)](1+g+p+g p))$ times previous period debt ratio, with $r=$ interest rate; $p=$ growth rate of GDP deflator; $g=$ real GDP growth rate; a = share of foreign-currency

denominated debt; and $\mathrm{e}=$ nominal exchange rate depreciation (measured by increase in local currency value of U.S. dollar).

${ }^{3}$ The real interest rate contribution is derived from the denominator in footnote $2 /$ as $r-\pi(1+g)$ and the real growth contribution as $-g$.

${ }^{4}$ The exchange rate contribution is derived from the numerator in footnote $2 /$ as ae $(1+r)$.

${ }^{5}$ Defined as public sector deficit, plus amortization of medium and long-term public sector debt, plus short-term debt at end of previous period.

${ }^{6}$ Derived as nominal interest expenditure divided by previous period debt stock.

${ }^{7}$ The key variables include real GDP growth; real interest rate; and primary balance in percent of GDP.

${ }^{8}$ Real depreciation is defined as nominal depreciation (measured by percentage fall in dollar value of local currency) minus domestic inflation (based on GDP deflator). 
Figure 1b. Public DSA: Bound Tests for Baseline Scenario (PC Restructuring and Nonconcessional Refinancing of Private Sector Creditors ${ }^{1}$

(Public debt in percent of GDP)

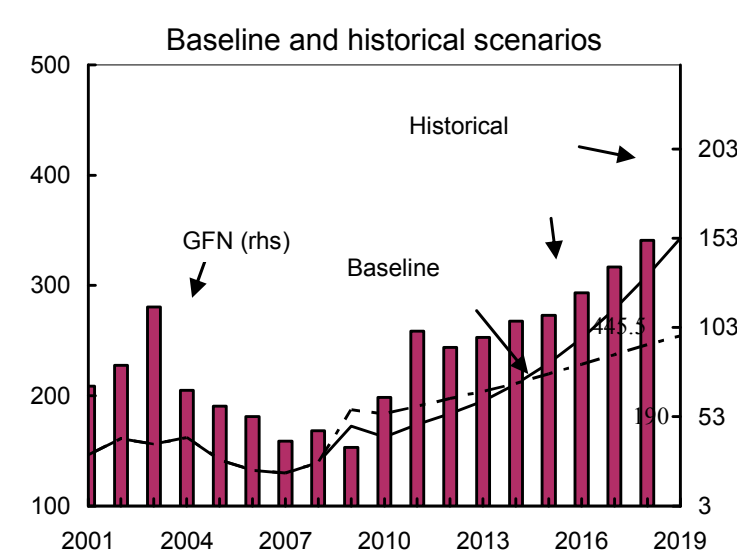

Growth shock (in percent per year)

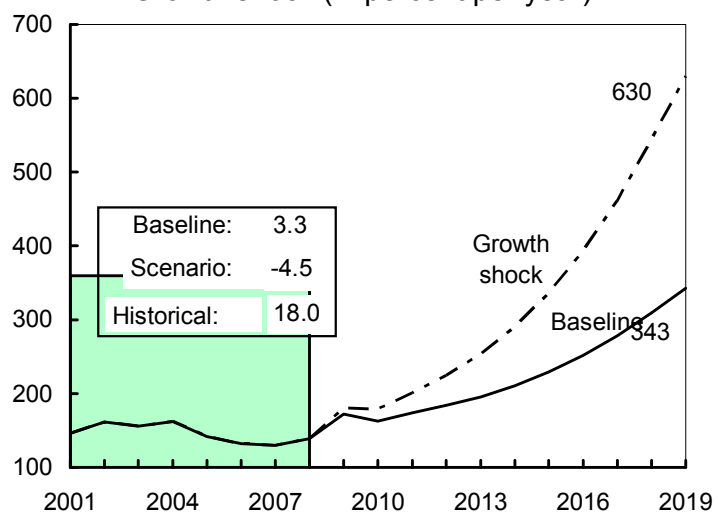

Combined shock $^{2}$

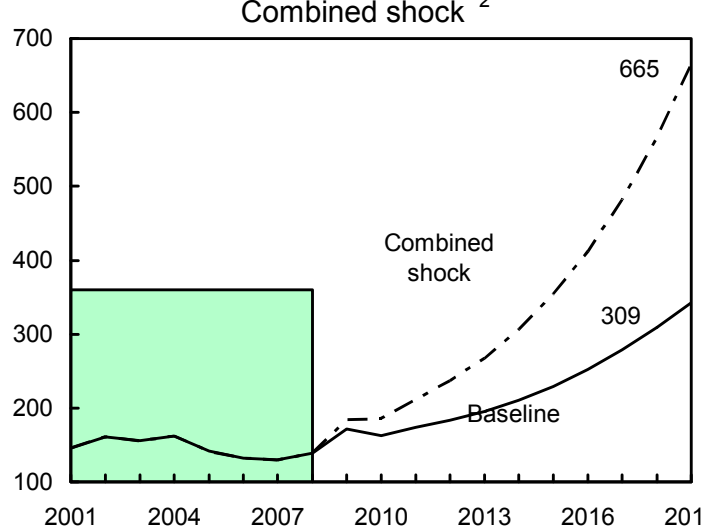

Interest rate shock (percent)

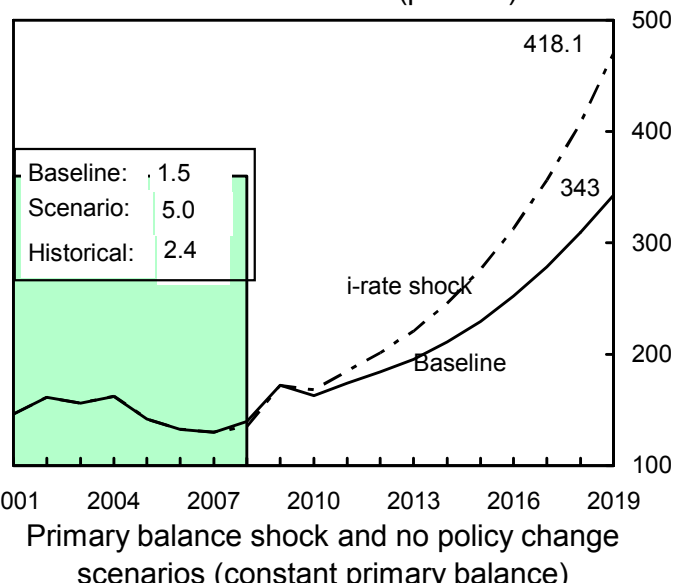

scenarios (constant primary balance)

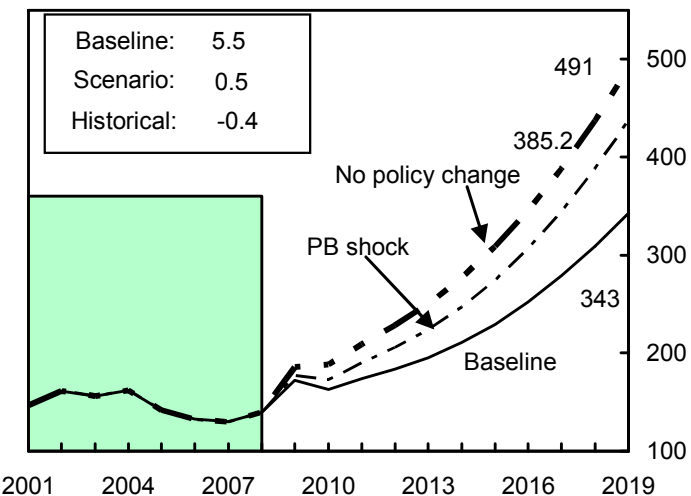

Real depreciation 3

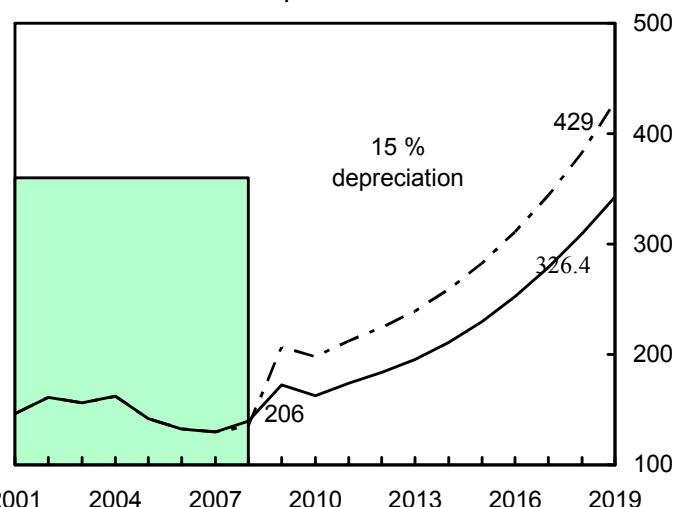

Sources: International Monetary Fund, Country desk data, and staff estimates.

${ }^{1}$ Shaded areas represent actual data. Individual shocks are permanent one standard deviation shocks (two s.d. for growth). Figures in the boxes represent average projections for the respective variables in the baseline and scenario being presented. Ten-year historical average for the variable is also shown.

${ }^{2}$ Permanent $1 / 4$ standard deviation shocks applied to real interest rate, growth rate, and primary balance.

${ }^{3}$ One-time additional real depreciation of 15 percent in 2009, with real depreciation defined as nominal depreciation (measured by percentage fall in dollar value of local currency) minus domestic inflation (based on GDP deflator). 


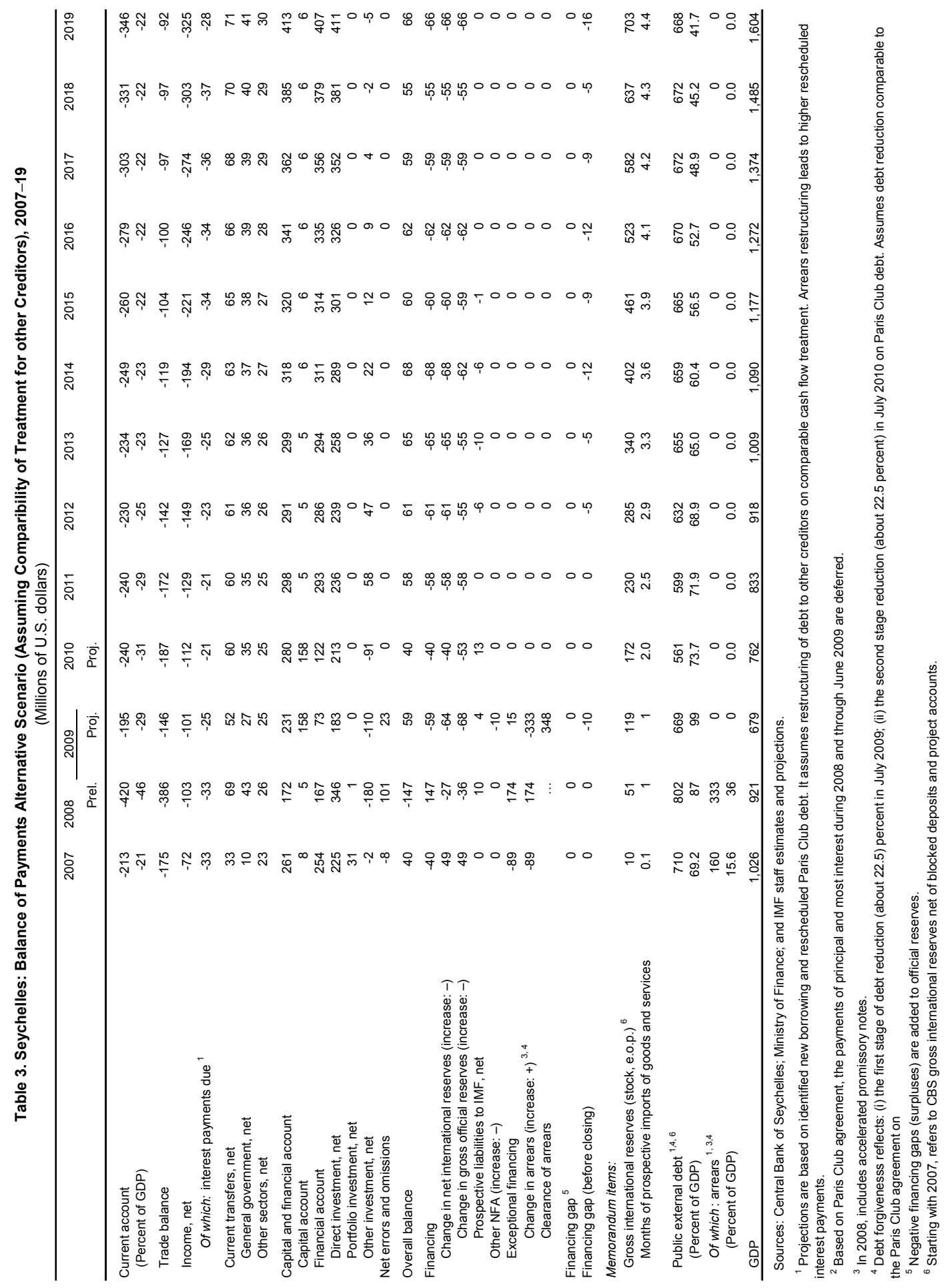


Table 3a. Seychelles: External Debt Sustainability Framework for Alternative Scenario (Assuming Comparibility of Treatment for other Creditors),2005-2019 (Percent of GDP, unless otherwise indicated)

\begin{tabular}{|c|c|c|c|c|c|c|c|c|c|c|c|c|c|c|c|}
\hline & \multicolumn{3}{|c|}{ Actual } & \multirow{2}{*}{$\begin{array}{r}\text { Prel } \\
2008 \\
\end{array}$} & \multicolumn{11}{|c|}{ Projections } \\
\hline & 2005 & 2006 & 2007 & & 2009 & 2010 & 2011 & 2012 & 2013 & 2014 & 2015 & 2016 & 2017 & 2018 & 2019 \\
\hline Baseline: external debt & 53.0 & 51.1 & 69.2 & 87.1 & 98.7 & 73.7 & 71.9 & 68.9 & 65.0 & 60.4 & 56.5 & 52.7 & 48.9 & 45.2 & 41.7 \\
\hline Change in external debt & -0.9 & -1.9 & 18.1 & 17.9 & 11.6 & -25.0 & -1.8 & -3.0 & -3.9 & -4.5 & -4.0 & -3.8 & -3.8 & -3.7 & -3.6 \\
\hline Identified external debt-creating flows $(4+8+9)$ & 6.0 & -5.7 & -1.4 & 8.7 & -14.9 & 0.4 & -2.9 & -4.1 & -5.5 & -6.6 & -6.3 & -6.3 & -6.0 & -5.6 & -6.2 \\
\hline Current account deficit, excluding interest payments & 18.3 & 12.1 & 17.7 & 43.4 & -7.5 & 27.9 & 25.7 & 22.3 & 20.7 & 20.2 & 19.2 & 19.3 & 19.4 & 19.8 & 19.8 \\
\hline Deficit in balance of goods and services & 18.0 & 13.2 & 17.0 & 41.9 & -9.2 & 24.5 & 20.6 & 15.5 & 12.6 & 10.9 & 8.8 & 7.8 & 7.0 & 6.5 & 5.8 \\
\hline Exports & 78.5 & 84.1 & 85.1 & 109.3 & 139.5 & 106.2 & 105.4 & 104.4 & 103.5 & 104.0 & 104.6 & 104.7 & 104.8 & 104.6 & 105.1 \\
\hline Imports & 96.5 & 97.3 & 102.1 & 151.1 & 130.4 & 130.8 & 126.0 & 119.9 & 116.1 & 114.9 & 113.5 & 112.6 & 111.9 & 111.2 & 110.8 \\
\hline Net non-debt creating capital inflows (negative) & -8.6 & -13.5 & -21.9 & -37.6 & -26.9 & -28.0 & -28.3 & -26.0 & -25.6 & -26.5 & -25.6 & -25.6 & -25.6 & -25.6 & -25.6 \\
\hline Automatic debt dynamics $^{1}$ & -3.7 & -4.3 & 2.8 & 2.9 & 19.5 & 0.5 & -0.3 & -0.5 & -0.7 & -0.3 & 0.0 & 0.1 & 0.2 & 0.2 & -0.3 \\
\hline Contribution from nominal interest rate & 0.7 & 1.1 & 3.1 & 2.2 & 6.9 & 3.6 & 3.1 & 2.8 & 2.5 & 2.7 & 2.8 & 2.7 & 2.7 & 2.5 & 1.8 \\
\hline Contribution from real GDP growth & -3.3 & -4.4 & -4.9 & 0.7 & 12.6 & -3.1 & -3.4 & -3.3 & -3.1 & -3.0 & -2.8 & -2.6 & -2.4 & -2.3 & -2.1 \\
\hline Contribution from price and exchange rate changes ${ }^{2}$ & -1.2 & -0.9 & 4.6 & 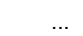 & 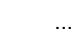 & & & $\ldots$ & $\ldots$ & & 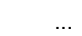 & $\ldots$ & $\ldots$ & $\ldots$ & \\
\hline Residual, incl. change in gross foreign assets $(2-3)^{3}$ & -6.9 & 3.8 & 19.5 & 9.2 & 26.5 & -25.4 & 1.1 & 1.1 & 1.6 & 2.1 & 2.4 & 2.5 & 2.3 & 2.0 & 2.6 \\
\hline External debt-to-exports ratio (in percent) & 67.5 & 60.8 & 81.3 & 79.7 & 70.7 & 69.4 & 68.2 & 65.9 & 62.8 & 58.1 & 54.0 & 50.3 & 46.6 & 43.2 & 39.7 \\
\hline Gross external financing need (in billions of US dollars) ${ }^{4}$ & 0.2 & 0.3 & 0.3 & 0.6 & 0.0 & 0.2 & 0.2 & 0.2 & 0.2 & 0.3 & 0.3 & 0.3 & 0.3 & 0.4 & 0.4 \\
\hline in percent of GDP & 24.7 & 25.1 & 27.3 & 66.1 & 1.6 & 31.4 & 28.8 & 25.1 & 23.7 & 23.8 & 23.2 & 23.1 & 23.4 & 23.7 & 23.1 \\
\hline
\end{tabular}

Key macroeconomic assumptions underlying baseline

Real GDP growth (percent)

Exchange rate appreciation (U.S. dollar value of local currency, change in percent) GDP deflator (change in domestic currency)

GDP deflator in U.S. dollars (change in percent)

Nominal external interest rate (percent)

Growth of exports (U.S. dollar terms, in percent)

Growth of imports (U.S. dollar terms, in percent)

Current account balance, excluding interest payments

Net non-debt creating capital inflows

$\begin{array}{rrrr}6.6 & 9.3 & 9.7 & -0.9 \\ 0.0 & -0.4 & -17.6 & -29.5 \\ 2.3 & 2.1 & 11.4 & 28.4 \\ 2.3 & 1.7 & -8.2 & -9.4 \\ 1.4 & 2.2 & 6.2 & 2.9 \\ 14.7 & 19.2 & 1.8 & 15.3 \\ 33.5 & 12.1 & 5.6 & 32.9 \\ -18.3 & -12.1 & -17.7 & -43.4 \\ 8.6 & 13.5 & 21.9 & 37.6\end{array}$

$\begin{array}{rrrrrrrrrrr}-10.7 & 3.5 & 5.0 & 5.0 & 5.0 & 5.0 & 5.0 & 5.0 & 5.0 & 5.0 & 5.0 \\ -37.3 & 4.3 & 1.7 & 1.7 & 1.7 & 0.0 & 0.0 & 0.0 & 0.0 & 0.0 & 0.0 \\ 31.4 & 4.0 & 2.4 & 3.1 & 2.9 & 2.9 & 2.9 & 2.9 & 2.9 & 2.9 & 2.9 \\ -17.6 & 8.5 & 4.1 & 4.9 & 4.7 & 2.9 & 2.9 & 2.9 & 2.9 & 2.9 & 2.9 \\ 5.8 & 4.1 & 4.6 & 4.2 & 3.9 & 4.5 & 5.1 & 5.1 & 5.4 & 5.5 & 4.2 \\ -6.0 & -14.5 & 8.5 & 9.1 & 9.0 & 8.5 & 8.7 & 8.2 & 8.1 & 7.8 & 8.5 \\ -36.5 & 12.7 & 5.4 & 4.8 & 6.4 & 6.9 & 6.7 & 7.2 & 7.4 & 7.4 & 7.7 \\ 7.5 & -27.9 & -25.7 & -22.3 & -20.7 & -20.2 & -19.2 & -19.3 & -19.4 & -19.8 & -19.8 \\ 26.9 & 28.0 & 28.3 & 26.0 & 25.6 & 26.5 & 25.6 & 25.6 & 25.6 & 25.6 & 25.6\end{array}$

Scenario with key variables at their historical averages 5 /

II. Stress Tests for External Debt Ratio

\section{B. Bound Tests}

B1. Nominal interest rate is at historical average plus one standard deviation

B2. Real GDP growth is at historical average minus one standard deviation

B3. Non-interest current account is at historical average minus one standard deviations

B4. Combination of B1-B3 using 1/2 standard deviation shocks

B5. One time 30 percent real depreciation in 2006

'Derived as $[r-g-\rho(1+g)+\varepsilon \alpha(1+r)] /(1+g+\rho+g \rho)$ times previous period debt stock, with $r=$ nominal effective interest rate on external debt; $\rho=$ change in domestic GDP deflator in US dollar terms, $g=$ real GDP growth rate,

$\varepsilon=$ nominal appreciation (increase in dollar value of domestic currency), and $\alpha=$ share of domestic-currency denominated debt in total external debt.

${ }^{2}$ The contribution from price and exchange rate changes is defined as $[-\rho(1+g)+\varepsilon \alpha(1+r)](1+g+\rho+g \rho)$ times previous period debt stock. $\rho$ increases with an appreciating domestic currency $(\varepsilon>0)$ and rising inflation (based on GDP deflator).

${ }^{3}$ For projection, line includes the impact of price and exchange rate changes.

Defined as current account deficit, plus amortization on medium- and long-term debt, plus short-term debt at end of previous period.

${ }^{5}$ The key variables include real GDP growth; nominal interest rate; dollar deflator growth; and both non-interest current account and non-debt inflows in percent of GDP. 
Figure 2a. External DSA: Bound Tests for Alternative Scenario (Assuming Comparibility of Treatment for other Creditors), 2003-19 ${ }^{1}$ (External debt in percent of GDP)
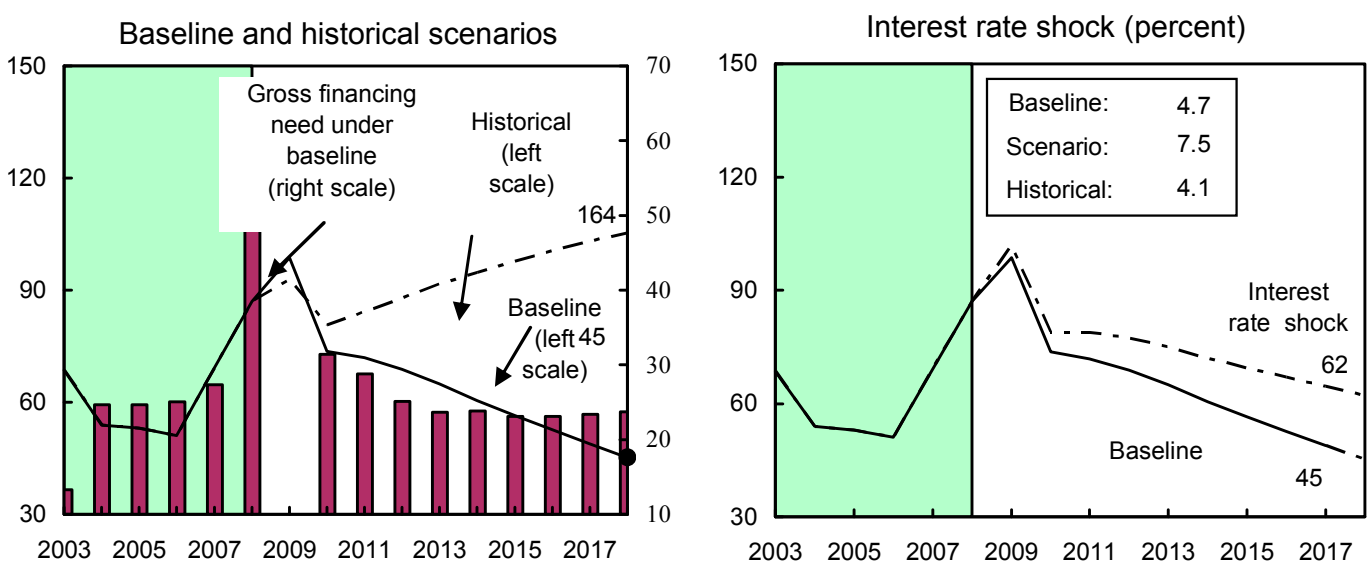

Growth shock

(percent per year)
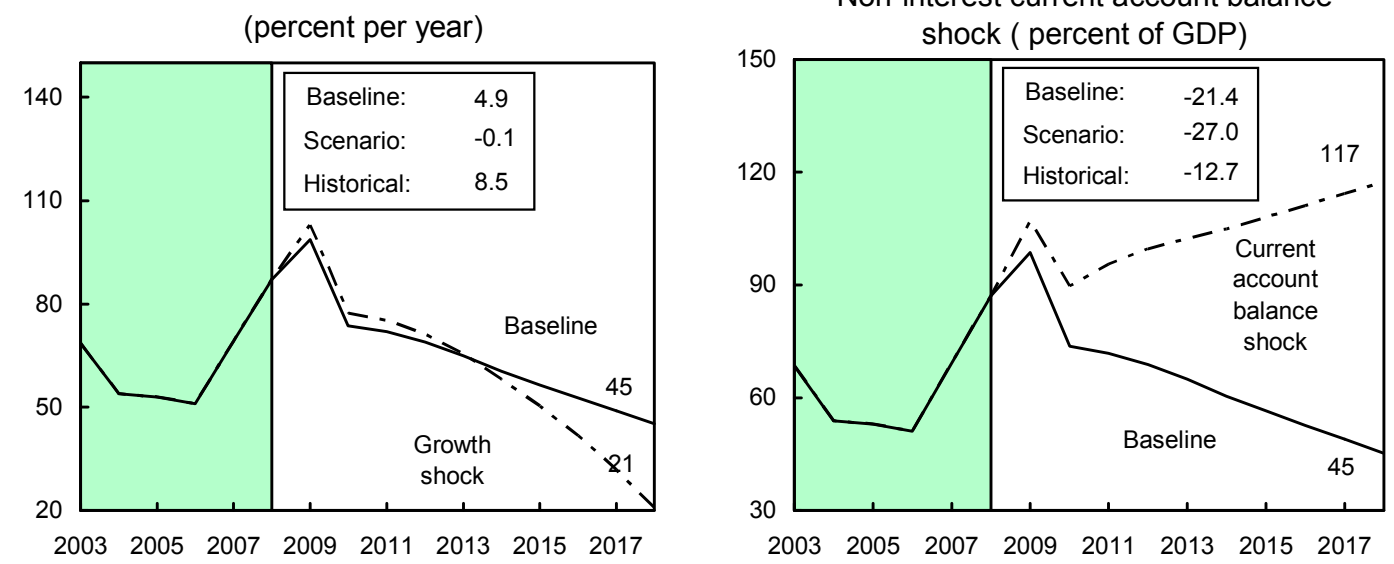

Combined shock ${ }^{2}$
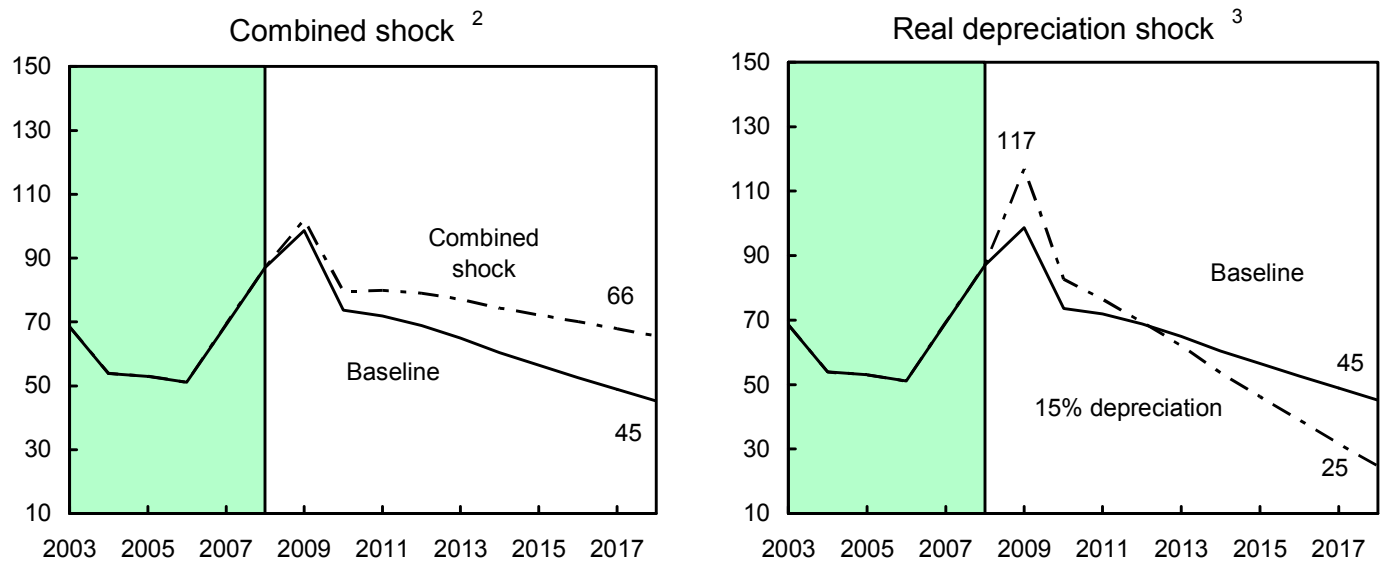

Sources: International Monetary Fund, Country desk data, and staff estimates.

${ }^{1}$ External financing gap is assumed to be closed by external borrowing at LIBOR plus $1300 \mathrm{bps}$, with 3 year maturity. Shaded areas represent actual data. Individual shocks are permanent one standard deviation shocks (two standard deviations for growth shock). Figures in the boxes represent average projections for the respective variables in the baseline and scenario being presented. Ten-year historical average for the variable is also shown.

${ }^{2}$ Permanent $1 / 4$ standard deviation shocks applied to real interest rate, growth rate, and current account balance.

${ }^{3}$ One-time real depreciation of additional 15 percent occurs in 2009. 
Table 3b. Seychelles: Public Sector Debt Sustainability Framework for Concessional Refinancing Scenario, 2006-19 (Percent of GDP, unless otherwise indicated)

\begin{tabular}{|c|c|c|c|c|c|c|c|c|c|c|c|c|c|c|c|}
\hline & \multirow[b]{2}{*}{2005} & \multicolumn{3}{|c|}{ Actual } & \multirow[b]{2}{*}{2009} & \multirow[b]{2}{*}{2010} & \multirow[b]{2}{*}{2011} & \multirow[b]{2}{*}{2012} & \multicolumn{2}{|c|}{ Projections } & \multirow[b]{2}{*}{2015} & \multirow[b]{2}{*}{2016} & \multirow[b]{2}{*}{2017} & \multirow[b]{2}{*}{2018} & \multirow[b]{2}{*}{2019} \\
\hline & & 2006 & 2007 & 2008 & & & & & 2013 & 2014 & & & & & \\
\hline $\begin{array}{l}\text { Public sector debt }{ }^{1} \\
\text { o/w foreign-currency denominated }\end{array}$ & $\begin{array}{r}141.8 \\
44.9\end{array}$ & $\begin{array}{r}132.4 \\
51.0\end{array}$ & $\begin{array}{r}129.8 \\
63.9\end{array}$ & $\begin{array}{r}139.4 \\
87.1\end{array}$ & $\begin{array}{r}140.9 \\
98.7\end{array}$ & $\begin{array}{r}113.8 \\
73.7\end{array}$ & $\begin{array}{r}107.7 \\
71.9\end{array}$ & $\begin{array}{r}100.9 \\
68.9\end{array}$ & $\begin{array}{l}94.0 \\
65.0\end{array}$ & $\begin{array}{l}87.3 \\
60.4\end{array}$ & $\begin{array}{l}81.5 \\
56.5\end{array}$ & $\begin{array}{l}76.6 \\
52.7\end{array}$ & $\begin{array}{l}71.9 \\
48.9\end{array}$ & $\begin{array}{l}67.0 \\
45.2\end{array}$ & $\begin{array}{l}\mathbf{6 2 . 5} \\
41.7\end{array}$ \\
\hline Change in public sector debt & -20.4 & -9.4 & -2.6 & 9.6 & 1.5 & -27.1 & -6.0 & -6.8 & -6.9 & -6.7 & -5.8 & -4.9 & -4.8 & -4.8 & -4.5 \\
\hline Identified debt-creating flows $(4+7+12)$ & -46.9 & -7.5 & -7.7 & -4.6 & 19.1 & -15.7 & -9.1 & -9.9 & -9.8 & -7.5 & -6.9 & -5.8 & -5.6 & -5.4 & -5.1 \\
\hline Primary deficit (negative means surplus) & -7.1 & 0.5 & 2.0 & -2.9 & -11.4 & -9.8 & -5.4 & -4.8 & -4.9 & -4.2 & -4.1 & -3.3 & -3.3 & -3.3 & -3.4 \\
\hline Revenue and grants & 40.6 & 41.2 & 32.2 & 35.5 & 35.7 & 34.3 & 33.6 & 33.7 & 34.3 & 34.1 & 34.9 & 34.4 & 34.7 & 34.7 & 34.8 \\
\hline Primary (noninterest) expenditure & 33.5 & 41.7 & 34.2 & 32.5 & 24.3 & 24.5 & 28.2 & 29.0 & 29.4 & 30.0 & 30.7 & 31.2 & 31.4 & 31.4 & 31.4 \\
\hline Automatic debt dynamics $^{2}$ & -38.6 & -9.3 & -7.8 & 1.3 & 34.3 & -4.2 & -2.7 & -4.2 & -4.1 & -2.5 & -2.0 & -1.8 & -1.5 & -1.4 & -1.1 \\
\hline Contribution from interest rate/growth differential ${ }^{3}$ & -38.6 & -9.4 & -17.4 & -20.6 & -12.3 & -0.2 & -1.5 & -3.1 & -3.0 & -2.5 & -2.0 & -1.8 & -1.5 & -1.4 & -1.1 \\
\hline Of which contribution from real interest rate & 3.1 & 2.4 & -6.9 & -21.5 & -25.0 & 4.4 & 3.8 & 1.9 & 1.7 & 1.9 & 2.1 & 2.0 & 2.0 & 1.9 & 2.0 \\
\hline Of which contribution from real GDP growth & -41.7 & -11.8 & -10.5 & 0.9 & 12.7 & -4.6 & -5.3 & -5.0 & -4.7 & -4.3 & -4.0 & -3.8 & -3.5 & -3.3 & -3.1 \\
\hline Contribution from exchange rate depreciation ${ }^{4}$ & 0.0 & 0.1 & 9.6 & 21.9 & 46.6 & -4.0 & -1.2 & -1.2 & -1.1 & 0.0 & 0.0 & 0.0 & 0.0 & 0.0 & 0.0 \\
\hline Denominator $=1+g+p+g p$ & 1.4 & 1.1 & 1.2 & 1.3 & 1.2 & 1.1 & 1.1 & 1.1 & 1.1 & 1.1 & 1.1 & 1.1 & 1.1 & 1.1 & 1.1 \\
\hline Other identified debt-creating flows & -1.3 & 1.2 & -2.0 & -2.9 & -3.8 & -1.8 & -0.9 & -0.9 & -0.9 & -0.8 & -0.8 & -0.7 & -0.7 & -0.7 & -0.6 \\
\hline Privatization receipts (negative) & -1.3 & -3.6 & -2.0 & -2.9 & -3.8 & -1.8 & -0.9 & -0.9 & -0.9 & -0.8 & -0.8 & -0.7 & -0.7 & -0.7 & -0.6 \\
\hline Recognition of implicit or contingent liabilities & 0.0 & 4.8 & 0.0 & 0.0 & 0.0 & 0.0 & 0.0 & 0.0 & 0.0 & 0.0 & 0.0 & 0.0 & 0.0 & 0.0 & 0.0 \\
\hline Other (specify, e.g. bank recapitalization) & 0.0 & 0.0 & 0.0 & 0.0 & 0.0 & 0.0 & 0.0 & 0.0 & 0.0 & 0.0 & 0.0 & 0.0 & 0.0 & 0.0 & 0.0 \\
\hline Residual, including asset changes (2-3) & 26.5 & -1.9 & 5.1 & 14.2 & -17.7 & -11.4 & 3.0 & 3.1 & 2.9 & 0.8 & 1.1 & 0.9 & 0.8 & 0.5 & 0.6 \\
\hline Public sector debt-to-revenue ratio ${ }^{1}$ & 349.5 & 321.6 & 403.1 & 392.9 & 394.6 & 331.8 & 320.5 & 299.0 & 274.0 & 255.7 & 233.8 & 222.4 & 207.0 & 193.3 & 180.3 \\
\hline Gross financing need ${ }^{5}$ & 59.1 & 53.1 & 39.4 & 46.1 & 24.1 & 32.7 & 28.9 & 24.5 & 21.1 & 17.4 & 15.9 & 15.5 & 14.5 & 13.9 & 14.9 \\
\hline Billions of U.S. dollars & 0.5 & 0.5 & 0.4 & 0.4 & 0.2 & 0.2 & 0.2 & 0.2 & 0.2 & 0.2 & 0.2 & 0.2 & 0.2 & 0.2 & 0.2 \\
\hline \multicolumn{16}{|l|}{ Key macroeconomic and fiscal aAssumptions } \\
\hline Real GDP growth (in percent) & 35.0 & 9.3 & 9.7 & -0.9 & -10.7 & 3.5 & 5.0 & 5.0 & 5.0 & 5.0 & 5.0 & 5.0 & 5.0 & 5.0 & 5.0 \\
\hline Average nominal interest rate on public debt (in percent) ${ }^{6}$ & 3.7 & 4.2 & 6.1 & 7.1 & 7.0 & 7.5 & 6.1 & 5.2 & 4.9 & 5.2 & 5.6 & 5.6 & 5.9 & 5.9 & 6.3 \\
\hline Average nominal interest rate on forex debt (in percent) ${ }^{6}$ & 1.7 & 2.6 & 7.5 & 4.4 & 5.8 & 4.1 & 4.6 & 4.2 & 3.9 & 4.5 & 5.1 & 5.1 & 5.4 & 5.5 & 4.2 \\
\hline Average real interest rate (nominal rate minus change in GDP deflator, in percent) & 2.9 & 2.1 & -5.3 & -21.3 & -24.4 & 3.5 & 3.7 & 2.1 & 2.0 & 2.3 & 2.7 & 2.7 & 3.0 & 3.0 & 3.4 \\
\hline Exchange rate (LC per US dollar) & 5.5 & 5.5 & 6.7 & 9.5 & 15.2 & 14.5 & 14.3 & 14.0 & 13.8 & 13.8 & 13.8 & 13.8 & 13.8 & 13.8 & 13.8 \\
\hline Nominal depreciation of local currency (LC per dollar) & 0.0 & 0.4 & 21.4 & 41.8 & 59.4 & -4.2 & -1.7 & -1.7 & -1.7 & 0.0 & 0.0 & 0.0 & 0.0 & 0.0 & 0.0 \\
\hline Nominal appreciation (increase in US dollar value of local currency, in percent) & 0.0 & -0.4 & -17.6 & -29.5 & -37.3 & 4.3 & 1.7 & 1.7 & 1.7 & 0.0 & 0.0 & 0.0 & 0.0 & 0.0 & 0.0 \\
\hline Inflation rate (GDP deflator, in percent) & 0.8 & 2.1 & 11.4 & 28.4 & 31.4 & 4.0 & 2.4 & 3.1 & 2.9 & 2.9 & 2.9 & 2.9 & 2.9 & 2.9 & 2.9 \\
\hline Growth of real primary spending (deflated by GDP deflator, in percent) & 0.4 & 36.0 & -10.0 & -5.8 & -33.3 & 4.4 & 20.6 & 8.1 & 6.7 & 6.8 & 7.8 & 6.5 & 5.8 & 5.0 & 5.0 \\
\hline Primary deficit & -7.1 & 0.5 & 2.0 & -2.9 & -11.4 & -9.8 & -5.4 & -4.8 & -4.9 & -4.2 & -4.1 & -3.3 & -3.3 & -3.3 & -3.4 \\
\hline \multicolumn{16}{|l|}{ A. Alternative scenarios } \\
\hline A1. Key variables are at their historical averages in $2008-12^{7}$ & & & & 139.4 & 165.1 & 153.6 & 159.4 & 165.4 & 171.6 & 177.5 & 184.1 & 190.7 & 197.4 & 204.0 & 211.1 \\
\hline A2. No policy change (constant primary balance) in $2008-12$ & & & & 139.4 & 154.3 & 138.6 & 139.5 & 138.2 & 136.7 & 135.1 & 134.4 & 133.6 & 133.1 & 132.4 & 132.3 \\
\hline \multicolumn{16}{|l|}{ B. Bound tests } \\
\hline B1. Real interest rate is at baseline plus one-half standard deviations & & & & 139.4 & 146.6 & 123.9 & 121.6 & 118.1 & 114.3 & 110.8 & 108.0 & 106.0 & 104.1 & 102.0 & 100.3 \\
\hline B2. Real GDP growth is at baseline minus two standard deviation & & & & 139.4 & 147.8 & 126.1 & 127.2 & 128.2 & 130.1 & 133.4 & 139.1 & 147.0 & 156.6 & 167.6 & 180.8 \\
\hline B3. Primary balance is at baseline minus one standard deviation & & & & 139.4 & 145.8 & 123.5 & 122.2 & 119.8 & 117.0 & 114.7 & 113.3 & 112.6 & 112.1 & 111.4 & 111.2 \\
\hline B4. Combination of B1-B3 using one-quarter standard deviation shocks & & & & 139.4 & 151.6 & 132.3 & 134.2 & 134.8 & 135.1 & 135.6 & 137.2 & 139.5 & 142.0 & 144.5 & 147.6 \\
\hline B5. One time 15 percent additional real depreciation in $2009^{8}$ & & & & 139.4 & 180.2 & 151.9 & 145.0 & 136.7 & 128.3 & 120.7 & 114.2 & 108.6 & 103.2 & 97.7 & 92.8 \\
\hline B6. 10 percent of GDP increase in other debt-creating flows in 2009 & & & & 139.4 & 150.9 & 123.5 & 117.2 & 110.0 & 102.7 & 95.8 & 89.8 & 84.7 & 79.8 & 74.8 & 70.2 \\
\hline
\end{tabular}

${ }_{1}^{1}$ Public sector covers non-finacial public sector and the IMF loan to the CBS. Debt is on a gross basis. External and domestic financing gaps are assumed to be closed by additional borrowing

${ }^{2}$ Derived as $[(r-p(1+g)-g+a e(1+r)] /(1+g+p+g p))$ times previous period debt ratio, with $r=$ interest rate; $p=$ growth rate of GDP deflator; $g=$ real GDP growth rate; $a=$ share of foreign-currency

denominated debt; and $\mathrm{e}=$ nominal exchange rate depreciation (measured by increase in local currency value of U.S. dollar).

${ }^{3}$ The real interest rate contribution is derived from the denominator in footnote $2 /$ as $r-\pi(1+g)$ and the real growth contribution as $-\mathrm{g}$.

${ }^{4}$ The exchange rate contribution is derived from the numerator in footnote $2 /$ as ae $(1+r)$.

${ }^{5}$ Defined as public sector deficit, plus amortization of medium and long-term public sector debt, plus short-term debt at end of previous period.

${ }^{6}$ Derived as nominal interest expenditure divided by previous period debt stock.

${ }^{7}$ The key variables include real GDP growth; real interest rate; and primary balance in percent of GDP.

${ }^{8}$ Real depreciation is defined as nominal depreciation (measured by percentage fall in dollar value of local currency) minus domestic inflation (based on GDP deflator). 
Figure 2b. Public DSA: Bound Tests for Restructuring Scenario ${ }^{1}$ (Public debt in percent of GDP)
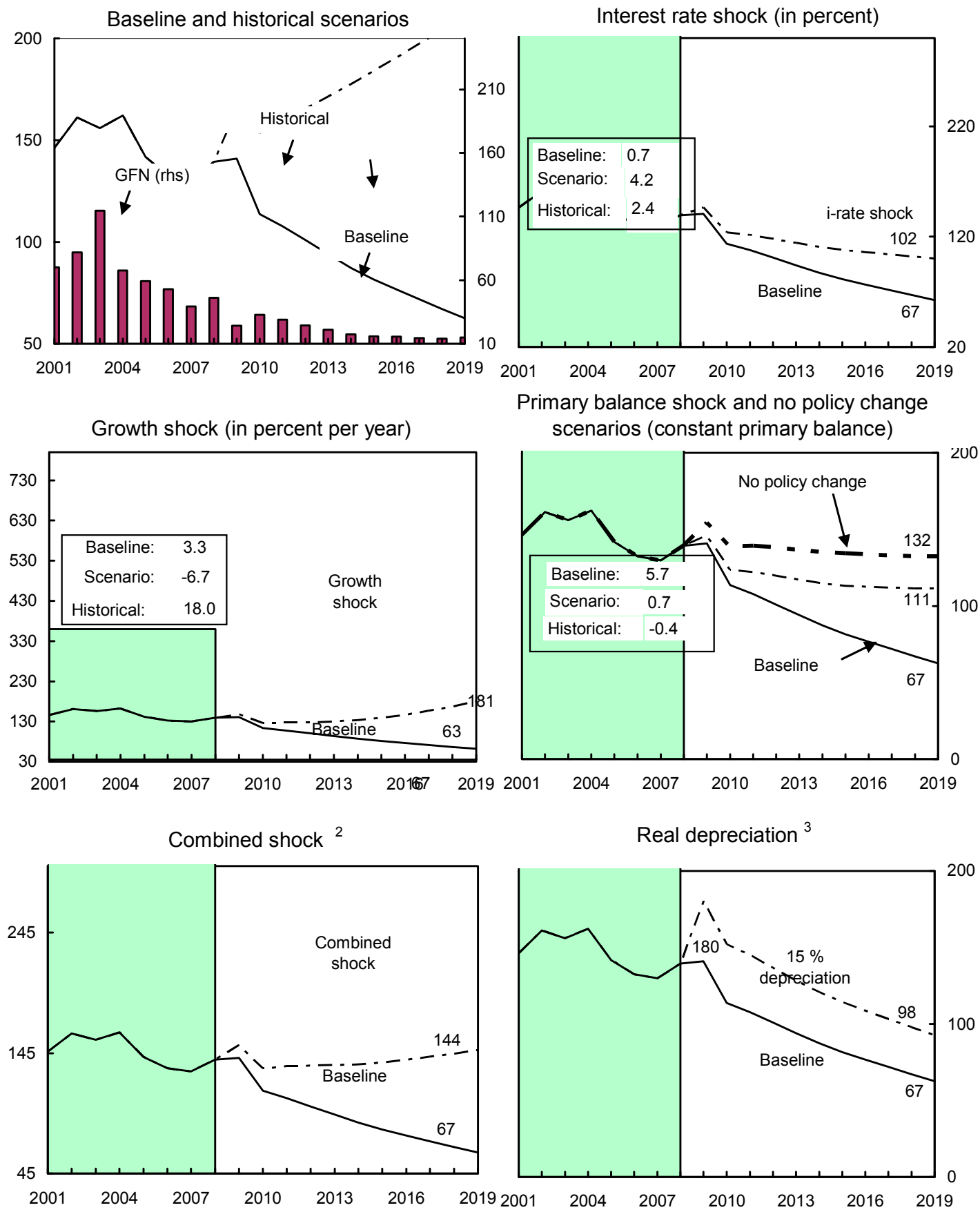

Sources: International Monetary Fund, Country desk data, and staff estimates.

${ }^{1}$ Shaded areas represent actual data. Individual shocks are permanent one standard deviation shocks (two s.d. for growth). Figures in the boxes represent average projections for the respective variables in the baseline and scenario being presented. Ten-year historical average for the variable is also shown.

${ }^{2}$ Permanent $1 / 4$ standard deviation shocks applied to real interest rate, growth rate, and primary balance.

${ }^{3}$ One-time additional real depreciation of 15 percent in 2009 , with real depreciation defined as nominal depreciation (measured by percentage fall in dollar value of local currency) minus domestic inflation (based on GDP deflator). 


\title{
INTERNATIONAL MONETARY FUND
}

\section{SEYCHELLES}

\section{Second Review Under the Stand-By Arrangement, Financing Assurances Review, and} Modification of Performance Criteria-Informational Annex

\author{
Prepared by the African Department \\ (In consultation with other departments) \\ Approved by Roger Nord (AFR) and Aasim Husain (SPR)
}

June 16, 2009

Contents

Page

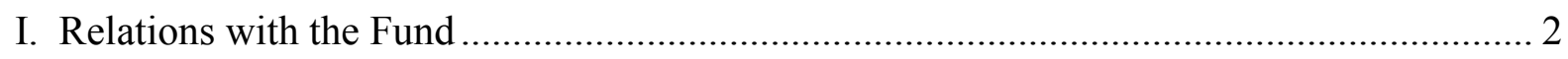

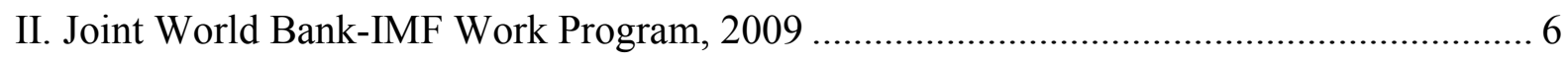

III.Relations with the African Development Bank Group ................................................. 8 


\section{SEYCHELLES: RELATIONS WITH THE FUND}

(As of May 31, 2009)

I. Membership Status: Joined 6/30/77. Article VIII.

\begin{tabular}{|c|c|c|}
\hline General Resources Account & SDR Million & $\%$ Quota \\
\hline Quota & 8.80 & 100.0 \\
\hline Fund holdings of currency & 15.84 & 179.97 \\
\hline Reserve Position in Fund & 0.00 & 0.04 \\
\hline
\end{tabular}

\begin{tabular}{|c|c|c|}
\hline II. SDR Department & $\underline{\text { SDR Million }}$ & $\%$ Allocations \\
\hline Net cumulative allocation & 0.41 & 100.0 \\
\hline Holdings & 0.02 & 5.20 \\
\hline
\end{tabular}

IV. Outstanding Purchases and $\quad 7.04 \quad 80.00$ Loans:

V. Financial Arrangements:

$\underline{\text { SDR Million }}$

Type Arrangement Expiration Amount Approved Amount Drawn

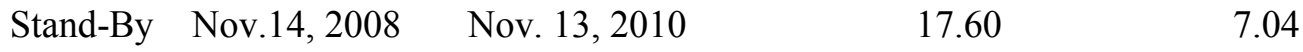

VI. Projected Obligations to the Fund (SDR Million: based on existing use of resources and present holdings of SDRs):

\begin{tabular}{l|llccc}
\hline & \multicolumn{5}{|c}{ Forthcoming } \\
\hline & $\mathbf{2 0 0 9}$ & $\mathbf{2 0 1 0}$ & $\mathbf{2 0 1 1}$ & $\mathbf{2 0 1 2}$ & $\mathbf{2 0 1 3}$ \\
Principal & & & & 3.30 & 3.52 \\
Charges/interest & 0.05 & 0.11 & 0.11 & 0.09 & 0.04 \\
Total & 0.05 & 0.11 & 0.11 & 3.39 & 3.56 \\
\hline
\end{tabular}


VII. Implementation of HIPC Initiative: Not applicable

\section{Safeguards Assessments:}

As part of the Stand-By Arrangement, a safeguards assessment mission was conducted in September 2008. It found high risks in all areas of the central bank (CBS) safeguards assessment framework, including significant weaknesses in financial reporting that were not identified by the external auditor and internal control vulnerabilities. The authorities are implementing remedial measures including steps monitored under the program. In late November the CBS appointed a new external auditor, PricewaterhouseCoopers, on a five-year contract. The authorities continue to make strong progress in addressing the areas of concern identified in the safeguards assessment, including a new central bank act that meets international best practice.

\section{Exchange Rate Arrangement:}

The authorities launched a comprehensive reform effort in November 2008, with reforms including a fundamental liberalization of the exchange regime. The exchange market liberalization resulted in all the elimination of restrictions on the making of payments and transfers for current international transactions that are subject to Fund approval under Article VIII Sections 2, 3, and 4. The exchange rate policy is a managed float. On May 31, 2009, US\$1 = SR 13.71 (mid rate).

\section{Article IV Consultations:}

Seychelles is currently under a two year Stand-By Arrangement, with quarterly reviews. 
XI. Technical Assistance (2004-March 2009):

\begin{tabular}{|c|c|c|c|}
\hline Department & Head of Mission & Subject & Date \\
\hline STA & Mr. Freeman & National Accounts/CPI & May/June 2005 \\
\hline MFD/LEG & Mr. Lonnberg & $\begin{array}{l}\text { Strengthening Capacity in } \\
\text { Critical Central Banking } \\
\text { Areas, including } \\
\text { AML/CFT-Legal, } \\
\text { Institutional, and } \\
\text { Supervisory Frameworks }\end{array}$ & July 2006 \\
\hline LEG & Mr. Beekarry & $\begin{array}{l}\text { Advise on AML/CFT } \\
\text { measures and the } \\
\text { establishment of the FIU }\end{array}$ & $\begin{array}{l}\text { September/October } \\
2006\end{array}$ \\
\hline STA & Mr. Alexander & $\begin{array}{l}\text { National } \\
\text { accounts/CPI/GDDS }\end{array}$ & $\begin{array}{l}\text { October/ } \\
\text { November } 2006\end{array}$ \\
\hline MCM & Mr. Bartholomew & $\begin{array}{l}\text { Multipurpose mission: } \\
\text { Monetary operations, } \\
\text { monetary research, } \\
\text { banking supervision, } \\
\text { payment systems, foreign } \\
\text { exchange markets, and } \\
\text { nonbank financial } \\
\text { institution supervision. }\end{array}$ & $\begin{array}{l}\text { November/ } \\
\text { December } 2007\end{array}$ \\
\hline STA & Mr. Dessart & $\begin{array}{l}\text { Dissemination of GDDS } \\
\text { National Summary Data } \\
\text { Page }\end{array}$ & April 2008 \\
\hline STA & Mr. Armknecht & Consumer price index & April 2008 \\
\hline MCM & Mr. Faulk & $\begin{array}{l}\text { Banking supervision, } \\
\text { drafting of new financial } \\
\text { sector related regulations }\end{array}$ & April 2008 \\
\hline MCM & Mr. Robotham & $\begin{array}{l}\text { National payment systems } \\
\text { project }\end{array}$ & May/June 2008 \\
\hline FAD & Mr. Khemani & $\begin{array}{l}\text { Strengthening expenditure } \\
\text { rationalization and budget } \\
\text { management }\end{array}$ & July 2008 \\
\hline LEG & Mr. Baban & $\begin{array}{l}\text { Exchange Rate and } \\
\text { Exchange Control } \\
\text { Regimes }\end{array}$ & September 2008 \\
\hline
\end{tabular}




\begin{tabular}{|c|c|c|c|}
\hline Department & Head of Mission & Subject & Date \\
\hline FIN & Mr. Hauge & Safeguards assessment & October 2008 \\
\hline FAD & Mr. Krelove & Reform of Tax Policy & $\begin{array}{l}\text { January/February } \\
2009\end{array}$ \\
\hline $\mathrm{MCM}$ & Mr. Bartholomew & $\begin{array}{l}\text { Central Bank Capacity } \\
\text { Building after the } 2008 \\
\text { Float }\end{array}$ & February 2009 \\
\hline LEG & Mr. Baban & $\begin{array}{l}\text { Reform of Exchange Rate } \\
\text { Law }\end{array}$ & May 2009 \\
\hline STA & Mr. Jones & $\begin{array}{l}\text { Government Finance } \\
\text { Statistics }\end{array}$ & May 2009 \\
\hline
\end{tabular}

XII. Resident Representative

None 


\section{Seychelles: Joint World BAnk-IMF Work Program, 2009}

(As of June 05, 2009)

\begin{tabular}{|c|c|c|c|}
\hline Title & Products & $\begin{array}{l}\text { Provisional timing of } \\
\text { mission }\end{array}$ & $\begin{array}{l}\text { Expected delivery } \\
\text { date }\end{array}$ \\
\hline \multicolumn{4}{|c|}{ A. Mutual Information on Relevant Work Programs } \\
\hline Bank & $\begin{array}{l}\text { A. Strategy and analytical work } \\
\text { Interim Strategy Note } \\
\text { Public Expenditure Review (PER) } \\
\text { Public Expenditure Review (PER, Phase 2) } \\
\text { Update to FIAS report (improving the business } \\
\text { environment and private sector development) } \\
\text { Joint Country Procurement Assessment Report } \\
\text { (CPAR) and Country Financial Accountability } \\
\text { Assessment (CFAA) } \\
\text { B. Ongoing and new projects } \\
\text { GEF grant implementation (tsunami reconstruction, } \\
\text { disaster management, fisheries capacity) } \\
\text { Development Policy Loan (DPL 1) } \\
\text { Development Policy Loan (DPL 2) }\end{array}$ & $\begin{array}{l}\text { November } 2008 \text { and } \\
\text { February } 2009 \\
\text { October } 2008 \\
\text { September } 2009 \\
\text { September } 2009 \\
\text { Ongoing Identification } \\
\text { mission: November } 2008 \\
\text { Identification mission: } \\
\text { November } 2009 \\
\text { November } 2009\end{array}$ & \begin{tabular}{|l} 
\\
April 2009 \\
February 2010 \\
February 2010 \\
\\
Ongoing
\end{tabular} \\
\hline Fund & $\begin{array}{l}\text { Program and surveillance work } \\
\text { 1. Article IV consultation and approval of 2-year Stand- } \\
\text { By Arrangement (SBA) } \\
\text { 2. Quarterly program and financing assurances } \\
\text { reviews under the SBA } \\
\text { First review } \\
\text { Second review } \\
\text { Third review } \\
\text { Fourth review (if needed) } \\
\text { 3. Discussion on medium-term structural reform } \\
\text { agenda that could be supported by a successor } \\
\text { arrangement under the EFF. } \\
\text { Technical assistance and capacity building } \\
\text { 1. FAD technical assistance mission on tax policy and } \\
\text { revenue administration review. } \\
\text { 2. FAD technical assistance mission on Public } \\
\text { Financial Management. } \\
\text { 3. MCM technical assistance on reforming the } \\
\text { monetary policy framework (long-term monetary } \\
\text { advisor, automation of interbank market, reserve } \\
\text { money management) strengthening bank supervision, } \\
\text { and CBS governance. } \\
\text { 4. MCM technical assistance on public debt } \\
\text { management. }\end{array}$ & $\begin{array}{l} \\
\text { February } 2009 \\
\text { May } 2009 \\
\text { August } 2009 \\
\text { October } 2009 \\
\text { October } 2009\end{array}$ & $\begin{array}{l}\text { March 27, } 2009 \\
\text { June 2009 } \\
\text { September } 2009 \\
\text { December } 2009 \\
\text { December } 2009\end{array}$ \\
\hline
\end{tabular}




\begin{tabular}{|l|l|l|l|}
\hline Title & Products & $\begin{array}{l}\text { Provisional timing of } \\
\text { mission }\end{array}$ & \multicolumn{1}{|c|}{$\begin{array}{l}\text { Expected } \\
\text { delivery date }\end{array}$} \\
\hline \multicolumn{2}{|c|}{ B. Requests for Work Program inputs } \\
\hline $\begin{array}{l}\text { Bank } \\
\text { request to } \\
\text { Fund }\end{array}$ & $\begin{array}{l}\text { 1. Medium-term macro-economic and fiscal framework } \\
\text { to inform Public Expenditure Reviews } \\
\text { Debt Sustainability Analysis }\end{array}$ & n.a. & Ongoing \\
\hline $\begin{array}{l}\text { Fund } \\
\text { request to } \\
\text { Bank }\end{array}$ & $\begin{array}{l}\text { 2. Assessment of the poverty impact of rupee float and } \\
\text { strategy on strengthening the social safety net and } \\
\text { enhancing targeting of social assistance. } \\
\text { 3. Policy note on parastatal reform and privatization. } \\
\text { 4. Strategy on improving business environment and } \\
\text { promoting private sector investment } \\
\text { 5. New wage grid proposal for public sector }\end{array}$ & $\begin{array}{l}\text { TBD } \\
\text { TBD }\end{array}$ & October 2009 \\
\hline \multicolumn{3}{|c|}{ C. Agreement on Joint Products and Missions } \\
\hline $\begin{array}{l}\text { Joint } \\
\text { products }\end{array}$ & $\begin{array}{l}\text { Joint mission to discuss a medium-term structural } \\
\text { reform agenda focusing on: social safety net targeting; } \\
\text { civil service reform, parastatal reform; and promoting } \\
\text { private sector investment }\end{array}$ & October 2009 (preliminary) & $\begin{array}{l}\text { December 2009 } \\
\text { (preliminary) }\end{array}$ \\
\hline
\end{tabular}




\title{
III. SeyChelles: Relations With THE AfRiCAn DEVElopMENT BANK GROUP
}

\author{
(As of June 5, 2009)
}

The African Development Bank Group commenced operations with the Republic of Seychelles in 1978 and has since approved 19 operations comprising 15 projects, one study and three lines of credit for the country. Cumulative approvals as of October 2008 (there were no approvals between 2001 and 2006 when the country was under sanctions) amounted to UA 84.87 million, ${ }^{1}$ of which 73 percent was from the African Development Bank (ADB), 14 percent from the Nigeria Trust Fund (NTF), and the remaining 13 percent from the African Development Fund (ADF) (Table 1). Reflecting government priorities, most of the operations were in the social sector (46 percent). This was followed by the financial sector (16 percent), agricultural sector (15 percent), transportation (13 percent), water supply and sanitation (10 percent) and industrial sector (1 percent). Before sanctions were imposed, about 76.8 percent of the total commitments on all approved loans had been disbursed and the balance of 23.2 percent was cancelled after sanctions were applied.

The country was under sanctions with the African Development Bank Group since February 16,2000 due to the arrears situation. The country cleared all the arrears (amounting to US\$46.75 million) in November 2006 and the African Development Bank Group consequently lifted all sanctions and started discussions on possible reengagement with the country. The process was boosted by the launching of a comprehensive reform programme by the Government in November 2008. Following this, an Interim Strategy Note was approved by the Board on April 22, 2009. The ISN covers 2009 and 2010 and focuses on a single pillar namely supporting the Government's reform programme to achieve sustainable growth. The lending programme during this period consists of a policy-based operation in the form of a budget support loan amounting to US \$ 20 million. The operation is under preparation and it is expected to be disbursed in two equal tranches of US \$ 10 million in 2009 and 2010. In addition, there is an ongoing non-lending programme under which the Bank Group is providing grant resources to the country, from the African Water Facility and the Middle Income Countries' (MIC) Technical Assistance Fund. Under the African Water Facility, a grant amounting to $€ 955,000$ was signed in May 2008 to finance the preparation of the Water Development Plan aimed at improved water management and governance, and identifying priority investment projects for funding, in order to meet Seychelles' water needs up to 2030. The study is expected to be completed by December 2009. Under the MIC Technical Assistance Fund two grants have been approved to finance (i) a study (amounting to UA 600,000) approved in December 2008 aimed mainly to support human resource

\footnotetext{
${ }^{1}$ UA $1=$ US\$ 1.54805 as at June 5, 2009.
} 
development, training and capacity building as well as the establishment of the Seychelles University Foundation; and (ii) a study (amounting to US\$ 500,000) approved in February 2009 to undertake the feasibility on a Submarine Cable System to link Seychelles to East Africa.

Table 1. Operations Summary as at 5 June 2009*

Operations since: 1978

Total Number of operations 19: 15 Projects, 1 Study; 3 Lines of Credit

\begin{tabular}{lcrrr}
\hline $\begin{array}{c}\text { Source of } \\
\text { Financing }\end{array}$ & $\begin{array}{c}\text { Commitments Percentage } \\
\text { (UA million) }\end{array}$ & $\begin{array}{c}\text { Share } \\
\text { Amoursed } \\
\text { AdU million) Disbursed }\end{array}$ \\
\hline ADB & 62.19 & 73 & 44.12 & 70.9 \\
ADF/TAF & 10.68 & 13 & 10.68 & 100.0 \\
NTF & 12.00 & 14 & 10.39 & 86.6 \\
Total & 84.87 & 100 & 65.19 & 76.8 \\
\hline
\end{tabular}

* Excludes the grants from the African Water Facility and the MIC Technical Assistance Facility due to their unique nature and small magnitude. 
Press Release No.09/246

International Monetary Fund

FOR IMMEDIATE RELEASE

Washington, D.C. 20431 USA

June 30, 2009

\section{IMF Executive Board Completes the Second Review Under Seychelles' Stand-By Arrangement And Approves US\$1.4 Million Disbursement}

The Executive Board of the International Monetary Fund (IMF) today completed the second review of Seychelles' economic performance under its two-year Stand-by Arrangement, and completed the latest quarterly financing assurances review. The Board's decision enables Seychelles to receive a disbursement in an amount equivalent to SDR 0.88 million (about US\$1.4 million).

The Executive Board also approved the modification of quarterly quantitative performance criteria, and agreed to establish a schedule of semi-annual program reviews, while maintaining quarterly quantitative performance criteria, and financing assurances reviews.

The two-year SDR 17.6 million (about US\$27.3 million) Stand-By Arrangement for Seychelles was approved on November 14, 2008 (see Press Release No. 08/282).

Following the Executive Board discussion, Mr. Takatoshi Kato, Deputy Managing Director and Acting Chair, stated:

'Seychelles' commitment to reform and prudent financial policies has brought about rapid progress on macroeconomic stabilization. The significant fiscal consolidation effort, backed by the new market-based monetary policy, has restored confidence in the Rupee and helped bring inflation down rapidly.

"Economic policies are geared toward consolidating this progress. The fiscal stance aims to support the disinflation objective and contribute to public debt sustainability, while preserving priority social spending. The market-based floating exchange rate regime is serving the economy well, and monetary policy should aim to maintain interest rates positive in real terms in order to anchor price stability. Efforts should continue to expand monetary policy instruments and modernize the legal and institutional framework for the financial sector.

"Structural reforms are needed to lay a firm foundation for sustained recovery. The authorities have strengthened tax administration and launched a fundamental tax policy 
reform, which are key to improving the investment climate, reducing economic distortions, and securing fiscal sustainability. Progress has also been made in strengthening public financial management and expenditure control. Further efforts in this direction, including to reinforce financial discipline and accountability in public enterprises, will be essential to ensuring fiscal sustainability and raising productivity.

"Good progress is being made with public external debt restructuring. Following the favorable agreement with the Paris Club, the authorities have shown good faith efforts to normalize relations with all creditor groups. In view of the high public debt level, the authorities are pursuing a debt restructuring with non-Paris Club creditors that is consistent with Seychelles' limited payments capacity. The authorities should reinforce their debt management capacity, supported by Fund technical assistance," said Mr. Kato. 


\section{Statement by Christopher Legg, Alternate Executive Director for Seychelles and Simon Duggan, Advisor to Executive Director June 30, 2009}

Our Seychellois authorities' determined adherence to their macroeconomic program is yielding results, with monetary and external stability now taking hold. Inflation fell to below 1 percent on an annualised basis in the first five months of this year and is expected to remain in the low single-digits through 2010. The exchange rate has appreciated over 20 percent against the US dollar since January - partially reversing the substantial depreciation that immediately followed the float; the accumulation of net international reserves continues to exceed program targets; and Seychelles' current account deficit is expected to decline sharply this year.

However, economic activity is expected to contract almost 11 percent in 2009 as the economy adjusts to tighter macroeconomic policies and with the global slowdown weighing heavily on the tourism sector and related industries. The threat of piracy has also had an adverse effect on commercial fishing activity in the region and, therefore, on Seychelles' tuna exports. Economic recovery is expected to take hold towards the end of this year, with real GDP growth forecast to be 3.5 percent in 2010 - a modest improvement on the staff projection at the first review and consistent with the rapid strengthening of Seychelles' macroeconomic frameworks.

Our authorities remain committed to maintaining prudent macroeconomic policies in accordance with program targets. The Government is pressing ahead with their ambitious structural reform agenda, focused on improving the efficiency of the tax system and reform of the public sector. Moreover, the Central Bank is making good progress towards addressing the recommendations of the IMF safeguards assessment.

\section{Fiscal Policy}

Fiscal policy is providing a strong nominal anchor to the economy, with the Government on track to achieve a fiscal tightening equivalent to almost 8 percentage points of GDP in 2009 . The primary fiscal surplus out-performed the revised program target in the first quarter of 2009, allowing the government to reduce its net domestic debt by an additional Rupee 500 million. The government is now forecasting a primary fiscal surplus of 11.4 percent of GDP in 2009, an improvement on the 9.8 percent estimate at the first review, and program targets have been revised up accordingly. The better-than-anticipated first quarter result and 2 associated decline in annual interest expenses has created the fiscal space for the government to meet urgent expenditure needs, specifically: reinforcement of border protection to combat the threat of piracy; the bring-forward of priority infrastructure projects; increased expenditure on social services; and a one-off injection to support the operations of the Public Utilities Corporation.

The government is maintaining a tight control over expenditures, including through continued public sector wage restraint, strengthened expenditure controls within key spending ministries and by improving financial management in state-owned enterprises (as 
detailed below). These efforts have been reinforced by the introduction of strengthened Public Procurement and Public Debt Acts and will be further supported by full implementation of a Treasury Single Account by end-September. Our authorities also remain committed to implementing the recommendations of a recent Public Expenditure Review conducted by the World Bank that identified scope for substantial savings in the health and education sectors, while retaining the quality of services.

\section{Monetary Policy}

Monetary policy has remained tight, with the Central Bank successfully controlling reserve money through market-based mechanisms. Market interest rates rose to over 30 percent at the start of the year and the exchange rate has appreciated in recent months. Notwithstanding a large decline in nominal interest rates in the second quarter, real interest rates remain high at around 10 percent. This has had the desired effect, with inflation coming down markedly from 38 percent at the start of the program to the low single-digits.

The outlook is for inflation to fall below 5 percent in year-on-year terms in 2010, consistent with continued tight monetary conditions, the forecast decline in economic activity and reduction in imported food and fuel prices. Importantly, there is no evidence of persistent inflationary pressures following the substantial exchange rate depreciation and related price level jump associated with the exchange rate depreciation in November. This has provided space for a measured counter-cyclical easing in monetary policy, consistent with the price stability objective recently enshrined in legislation under the new Central Bank Act.

\section{Financial Sector}

With financial stabilization now taking hold, Seychelles' banks are emerging from a period of global and domestic financial sector turbulence in a strong position overall. The banking sector weathered the severe interest and exchange rate shock that followed the move to market-based mechanisms well. Banking sector capitalization exceeds standard norms, profitability remains strong and the percentage of non-performing loans remains low. In addition, the Central Bank's stress tests - conducted on the basis of new capitalization norms introduced in May and more stringent provisioning requirements introduced in June - show that the financial sector is well-placed to withstand further substantial shocks. Nonetheless, our authorities are attuned to the potential for credit risks to increase with declining economic activity and higher unemployment and remain vigilant in their supervision of banking sector balance sheets. The Central Bank is also working on contingency guidelines specifying 3 prompt and corrective actions to be taken in the unlikely event of severe market stress.

\section{Structural Reforms}

Earlier this month the Government announced a comprehensive tax reform strategy. The central pillars are: transforming the current goods and services tax (GST) into a value-added tax (VAT); replacing the current social security contributions scheme and other taxes on personal earnings with a personal income tax system; and overhauling the business tax regime through lowering/eliminating the threshold, rate and accelerated capital depreciation 
schedules towards international standards. A central element of these reforms is the elimination of tax concessions provided to the tourism, fisheries and agriculture industries, bringing these sectors into the new tax regime and leveling the playing field. Implementation of the Government's tax reform strategy will be staged, with the income tax measures (business and personal) to be introduced with the 2010 Budget and the VAT expected to be fully operational by January 2012 (with IMF technical support). The government's tax reform strategy is expected to be revenue neutral in 2009, with tax receipts expected to increase over time in line with nominal income growth. Our authorities are taking parallel measures to strengthen tax administration, both in terms of staffing and procedures, and will receive IMF technical assistance in this area shortly.

The second pillar of the Government's structural reform agenda is rationalization and reform of the public sector. In 2008, the government privatized several state-owned enterprises, reduced the number of civil service employees by 17 percent and outsourced a range of activities that could be provided more efficiently by the private sector. The next phase of the government's public sector reforms is a strategic assessment of all state-owned enterprises to evaluate the costs and benefits of continued government ownership. For those enterprises where continued government ownership is considered appropriate, the government will take measures to maximise the value of its shareholding and reduce risks to the public sector balance sheet. Informing this process are financial audits (by a major international accounting firm) and management audits (by an international expert working within the Ministry of Finance) of each public sector enterprise to assess current business practices against international financial, governance and management standards. These audits will be completed towards the end of this year and the government is committed to responding decisively to the findings.

\section{Public Debt Restructuring Strategy}

Our authorities are continuing their good faith efforts to achieve a comprehensive public external debt restructuring, consistent with their medium-term payments capacity and the Fund's lending into arrears policy. Agreement has now been reached with Paris Club Creditors and South Africa on an exceptional treatment of the government's debts under the Evian approach. Negotiations are continuing with other creditors and our authorities working towards the launch of a debt exchange offer before the end of this year, guided by their commitment to inter-creditor equity.

Our authorities are also making progress on a medium-term debt management strategy to 4 reduce the cost and risk of public debt, while taking into account monetary and fiscal policy objectives. They are aiming to finalize the strategy by November, with the assistance of the Fund's Monetary and Capital Markets Department.

\section{Program Performance}

Progress on macroeconomic stabilization is underpinned by our authorities' strong program performance. They comfortably met all of the end-March quantitative targets and have implemented the two structural benchmarks for the second review. The Seychelles Revenue Authority also recently completed tax audits of the 20 largest firms, meeting the structural 
performance criterion for the third review prior to the end-June deadline. Our authorities support staff's proposal that future program reviews be conducted on a semi-annual basis, consistent with the strength of their program performance and the stabilization of macroeconomic conditions.

As stated in President Michel's letter of intent, Seychelles will request a 3-year arrangement under the Extended Fund Facility (EFF) to replace their current Stand-By Arrangement in late 2009. Our Seychellois authorities are actively working with the IMF, World Bank and African Development Bank to elaborate a comprehensive medium-term reform program, where the EFF provides the appropriate macroeconomic framework and financing structure through which to address their medium-term challenges.

Finally, our Seychellois authorities would like to express their gratitude to Management for their support and the mission chief and his team for their continued hard work in helping to shape Seychelles' economic reform agenda. 